\title{
THE PARKES Hi ZONE OF AVOIDANCE SURVEY
}

\author{
L. Staveley-Smith ${ }^{1,2}$, R. C. KraAn-Korteweg ${ }^{3}$, A. C. Schröder ${ }^{4}$, P. A. Henning ${ }^{5}$, B. S. Koribalski ${ }^{6}$, I. M. Stewart ${ }^{7}$, And \\ G. HEALD 8,9 \\ ${ }^{1}$ International Centre for Radio Astronomy Research, University of Western Australia, Crawley, WA 6009, Australia \\ ${ }^{2}$ ARC Centre of Excellence for All-sky Astrophysics, Australia \\ ${ }^{3}$ Astrophysics, Cosmology and Gravity Centre (ACGC), Department of Astronomy, University of Cape Town, Private Bag X3, Rondebosch 7701, South Africa \\ ${ }^{4}$ South African Astronomical Observatory, P.O. Box 9, Observatory 7935, Cape Town, South Africa \\ ${ }^{5}$ Department of Physics and Astronomy, University of New Mexico, 1919 Lomas Blvd. NE, Albuquerque, NM 87131, USA \\ ${ }^{6}$ CSIRO Astronomy and Space Science, Australia Telescope National Facility, P.O. Box 76, Epping, NSW 1710, Australia \\ ${ }^{7}$ Sterrewacht Leiden, University of Leiden, Niels Bohrweg 2, 2333 CA Leiden, The Netherlands \\ ${ }^{8}$ ASTRON, The Netherlands Institute for Radio Astronomy, Postbus 2, 7990 AA, Dwingeloo, The Netherlands \\ ${ }_{9}$ Kapteyn Astronomical Institute, Postbus 800, $9700 \mathrm{AV}$, Groningen, The Netherlands \\ Received 2015 July 24; accepted 2015 September 26; published 2016 February 9
}

\begin{abstract}
A blind $\mathrm{H}_{\text {I }}$ survey of the extragalactic sky behind the southern Milky Way has been conducted with the multibeam receiver on the $64 \mathrm{~m}$ Parkes radio telescope. The survey covers the Galactic longitude range $212^{\circ}<\ell<36^{\circ}$ and Galactic latitudes $|b|<5^{\circ}$ to an rms sensitivity of 6 mJy per beam per $27 \mathrm{~km} \mathrm{~s}^{-1}$ channel and yields 883 galaxies to a recessional velocity of $12,000 \mathrm{~km} \mathrm{~s}^{-1}$. The survey covers the sky within the H I Parkes All-Sky Survey (HIPASS) area to greater sensitivity, finding lower $\mathrm{H}_{\mathrm{I}}$ mass galaxies at all distances, and probing more completely the large-scale structures at and beyond the distance of the Great Attractor. Fifty-one percent of the H I detections have an optical/near-infrared (NIR) counterpart in the literature. A further $27 \%$ have new counterparts found in existing, or newly obtained, optical/NIR images. The counterpart rate drops in regions of high foreground stellar crowding and extinction, and for low $\mathrm{H}$ I mass objects. Only $8 \%$ of all counterparts have a previous optical redshift measurement. The $\mathrm{H}_{\mathrm{I}}$ sources are found independently of Galactic extinction, although the detection rate drops in regions of high Galactic continuum. The survey is incomplete below a flux integral of approximately $3.1 \mathrm{Jy} \mathrm{km} \mathrm{s}{ }^{-1}$ and mean flux density of approximately $21 \mathrm{mJy}$, with $75 \%$ and $81 \%$ of galaxies being above these limits, respectively. Taking into account dependence on both flux and velocity width, and constructing a scaled dependence on the flux integral limit with velocity width $\left(w^{0.74}\right)$, completeness limits of $2.8 \mathrm{Jy} \mathrm{km} \mathrm{s}{ }^{-1}$ and $17 \mathrm{mJy}$ are determined, with $92 \%$ of sources above these limits. A notable new galaxy is HIZOA J1353-58, a possible companion to the Circinus galaxy. Merging this catalog with the similarly conducted northern extension, largescale structures are delineated, including those within the Puppis and Great Attractor regions and the Local Void. Several newly identified structures are revealed here for the first time. Three new galaxy concentrations (NW1, NW2, and NW3) are key in confirming the diagonal crossing of the Great Attractor Wall between the Norma Cluster and the CIZA J1324.7-5736 cluster. Further contributors to the general mass overdensity in that area are two new clusters (CW1 and CW2) in the nearer Centaurus Wall, one of which forms part of the striking $180^{\circ}\left(100 \mathrm{~h}^{-1} \mathrm{Mpc}\right)$ long filament that dominates the southern sky at velocities of $\sim 3000 \mathrm{~km} \mathrm{~s}^{-1}$, and the suggestion of a further wall at the Great Attractor distance at slightly higher longitudes.
\end{abstract}

Key words: galaxies: distances and redshifts - galaxies: fundamental parameters - large-scale structure of universe - surveys

Supporting material: figure set, machine-readable and VO tables

\section{INTRODUCTION}

In addition to serving as a direct probe of neutral hydrogen gas, observations of the $21 \mathrm{~cm}$ line of $\mathrm{H}$ I allow the detection of galaxies through the thickest Galactic obscuration and the presence of the highest foreground stellar confusion, which mask galaxies at optical/IR wavelengths. The historical Zone of Avoidance (ZOA) has prevented study of the distribution of galaxies behind the Milky Way and is most pronounced in the optical. Dedicated low-Galactic-latitude optical searches and surveys of galaxies in the infrared have narrowed the ZOA, but still fail to detect galaxies where Galactic emission, dust obscuration, and stellar confusion prevent the recognition of background galaxies. The near-infrared (NIR) has been particularly fruitful for finding large numbers of low-latitude galaxies. However, the most homogeneous NIR wide-angle redshift survey, the Two Micron All Sky Survey (2MASS) Redshift Survey (Huchra et al. 2012), is still not an all-sky survey, as it retains a gap of $5^{\circ}-8^{\circ}$ around the Galactic plane. While this does not affect our understanding of galaxy populations, as there is no reason to think that galaxies in the ZOA are in any way different from high-latitude ones, the lack of information in the ZOA contributes uncertainty to our understanding of dynamics in the local universe. Controversial results persist for the apex and convergence radius for the cosmic microwave background dipole from galaxy redshift and peculiar velocity surveys (e.g., Erdogdu et al. 2006; Watkins et al. 2009; Lavaux et al. 2010; Lavaux \& Hudson 2011; Courtois et al. 2012; Ma et al. 2012; Springob et al. 2014). While insufficient depth in redshift space of the relevant data sets (e.g., optical, 2MASS, IRAS, galaxy clusters) may be a factor, the incomplete mapping of the $\mathrm{ZOA}$ is also important (e.g., Kraan-Korteweg \& Lahav 2000; Loeb \& Narayan 2008).

Beyond the ability to find galaxies in regions of arbitrarily high extinction and stellar confusion, using the $21 \mathrm{~cm}$ line of 
$\mathrm{H}$ I to detect galaxies has the added benefit of immediate redshift measurement, eliminating the need that two-dimensional imaging surveys have for follow-up observations to obtain redshifts. This blind $\mathrm{H}_{\mathrm{I}}$ survey technique for finding hidden galaxies was pioneered by Kerr \& Henning (1987), but technology allowing large areas of the sky to be surveyed at $21 \mathrm{~cm}$ became available only relatively recently, with L-band multibeam receivers installed on the Parkes, Arecibo, Jodrell Bank Lovell, and Effelsberg radio telescopes.

We report here on a $21 \mathrm{~cm} \mathrm{H}$ I survey of the southern hemisphere ZOA, fully covering the area $212^{\circ}<\ell<36^{\circ}$; $|b|<5^{\circ}$ to a sensitivity of $6 \mathrm{mJy}$ at velocity resolution of $27 \mathrm{~km} \mathrm{~s}^{-1}$ with the $64 \mathrm{~m}$ Parkes radio telescope. We refer to this survey as HIZOA-S. A shallow survey of this area, with partial data and much lower sensitivity $(15 \mathrm{mJy})$, was presented by Henning et al. (2000), and an intermediate-depth study of the Great Attractor region was conducted by Juraszek et al. (2000), with rms $20 \mathrm{mJy}$ at slightly better velocity resolution. Two extensions to the north $\left(\ell=36^{\circ}-52^{\circ}\right.$ and $\left.\ell=196^{\circ}-212^{\circ}\right)$ have been studied at identical sensitivity to HIZOA-S by Donley et al. (2005). We refer to this as HIZOA-N. A region of the Galactic bulge, above and below our latitude range around the Galactic center, has also been surveyed to higher Galactic latitude, but has not been fully analyzed yet. Preliminary descriptions appear in Shafi (2008) and Kraan-Korteweg et al. (2008). The current work presents the data for the main fullsensitivity southern ZOA survey, HIZOA-S. The discussion of large-scale structures (Section 7) makes use of the combined southern and northern data. We refer to the combined sample as HIZOA. The Hi Parkes All-Sky Survey (HIPASS; Meyer et al. 2004) also covers the area, but to 2-3 times lower sensitivity than the current work, and it is unable to define detailed structure at Great Attractor distances.

An early fully sampled, blind, but shallow $\mathrm{H}_{\mathrm{I}}$ survey (Koribalski et al. 2004) has delineated local large-scale structures at low Galactic latitudes. Further, pointed $21 \mathrm{~cm}$ observations of partially obscured galaxies have revealed largescale structures in selected regions (Kraan-Korteweg et al. 2002; Schröder et al. 2009), but the current work covers the entire southern ZOA, over $1800 \mathrm{deg}^{2}$, in a blind, deep, and complete fashion. The remainder of the great circle of the ZOA in the northern hemisphere is now being surveyed by the Arecibo (Henning et al. 2010; McIntyre et al. 2015) and Effelsberg (Kerp et al. 2011) radio telescopes. There are also ongoing efforts to uncover the three-dimensional distribution of partially obscured galaxies with pointed $21 \mathrm{~cm}$ observations of selected galaxies visible in the NIR with the Nançay radio telescope (van Driel et al. 2009; Ramatsoku et al. 2014). The combination of $\mathrm{H}_{\mathrm{I}}$ and deep NIR imaging in regions of modest extinction and stellar confusion allows determination of peculiar velocities via the Tully-Fisher relation and thus provides information on galaxy flows. Such surveys are possible in the ZOA with the advent of large $\mathrm{H}_{\text {I }}$ surveys, such as the current work, and follow-up NIR imaging of the counterparts of the HIZOA galaxies (Williams et al. 2014; Said et al. 2015). In the future, all-sky $\mathrm{H}_{\mathrm{I}}$ surveys, using the Australian Square Kilometre Array Pathfinder (ASKAP; Johnston et al. 2007b; Koribalski 2012) and the APERture Tile In Focus system on the Westerbork telescope in the north (APERTIF; Verheijen et al. 2009), will allow comprehensive and complete mapping of large-scale structures as revealed by neutral hydrogen. Preparatory work is already under way for
Table 1

HIZOA Survey Parameters

\begin{tabular}{lcc}
\hline \hline Parameter & Value & Unit \\
\hline Galactic longitude & $212^{\circ}<\ell<36^{\circ}$ & $\ldots$ \\
Galactic latitude & $-5^{\circ}<b<5^{\circ}$ & $\ldots$ \\
Velocity range & $-1,280<c z<12,740$ & $\mathrm{~km} \mathrm{~s}^{-1}$ \\
Velocity resolution & 27 & $\mathrm{~km} \mathrm{~s}^{-1}$ \\
Scan rate & 1.0 & degree minute $^{-1}$ \\
Beam size (FWHP) & 15.5 & arcmin \\
Correlator cycle & 5 & $\mathrm{~s}$ \\
Integration time per beam & 2100 & $\mathrm{~s}$ \\
Cube rms & 6 & $\mathrm{mJy}$ \\
\hline
\end{tabular}

such large interferometric ZOA surveys; for instance, the Westerbork Synthesis Radio Telescope (WSRT) has been used for a deep ZOA mosaic with a field of view close to that of the forthcoming APERTIF system. Ultimately, the SKA itself will extend such $\mathrm{H}_{\text {I }}$ surveys further in redshift.

In Section 2, we present details of the observations and data reduction for the survey. In Section 3, we describe the sample compilation and $\mathrm{H}$ I parameterization. In Section 4, H I properties of the sample are presented, and multiwavelength counterparts are described in Section 5. Section 6 presents the survey's completeness, reliability, and parameter accuracy. In Section 7, new large-scale structures and their relationships to high-latitude structures are discussed. We present conclusions in Section 8.

\section{OBSERVATIONS AND DATA REDUCTION}

\subsection{Multibeam Observations}

The observations described here were taken with the $21 \mathrm{~cm}$ multibeam receiver (Staveley-Smith et al. 1996) at the Parkes telescope between 1997 March 22 and 2000 June 8, contemporaneously with the southern component of HIPASS. The observations cover the Galactic longitude range $212^{\circ}<\ell<36^{\circ}$ in 23 separate regions, each $8^{\circ}$ wide in longitude, and cover the latitude range $5^{\circ}>b>-5^{\circ}$ with almost uniform sensitivity. As noted above, later observations (Donley et al. 2005) extended the longitude range by $16^{\circ}$ in each direction (HIZOA-N). The observational parameters are identical to those described in Donley et al. (2005), but are summarized in Table 1 for completeness.

Each of the 23 longitude regions $\left(8^{\circ}\right.$ in longitude by $10^{\circ}$ in latitude) was scanned in raster fashion by the telescope at a rate of $1^{\circ}$ minute ${ }^{-1}$, in a direction of increasing, or decreasing, Galactic longitude. Each scan therefore lasted 8 minutes plus overlap and turnaround time. The central beam was scanned at constant latitude; however, to minimize bandpass effects, the receiver was not rotated ("parallactified") during the scan. The other 12 beams tended therefore to deviate slightly from constant latitude. The receiver rotation was adjusted such that the feed rotation was approximately $15^{\circ}$ from the direction of the scan at the midpoint of each observation (Staveley-Smith 1997). This ensured almost-Nyquist coverage of the sky even for a single scan, covering a region $1^{\circ} .7 \times 8^{\circ}$ in size. However, for sensitivity reasons, each of the 23 regions was raster scanned 425 times, giving a total integration time of approximately $2100 \mathrm{~s}$ for each point of the sky (with the actual value dependent on gridding strategy) and an rms noise of $6 \mathrm{mJy}$ beam $^{-1}$. The observations were conducted at night over 
a period of 3 years, and there was considerable redundancy in the data. Each point in the sky was visited hundreds of times, allowing mitigation of the already low levels of radio frequency interference (RFI) in this band. For more details, see Meyer et al. (2004). The main issue encountered was confusion with Galactic recombination lines (see Meyer et al. 2004; Alves et al. 2015), which, being diffuse and associated with radio continuum emission, was straightforward to recognize in the image domain.

The central observing frequency was $1394.5 \mathrm{MHz}$ with a bandwidth of $64 \mathrm{MHz}$ and a channel spacing of $62.5 \mathrm{kHz}$. This corresponds to a velocity range of $-1280<c z<12,740 \mathrm{~km} \mathrm{~s}^{-1}$ and a channel spacing of $13.2 \mathrm{~km} \mathrm{~s}^{-1}$ at zero redshift. After Hanning smoothing, the velocity resolution was $27 \mathrm{~km} \mathrm{~s}^{-1}$. The correlator integration time was $5 \mathrm{~s}$, resulting in negligible smearing along the scan direction. Two orthogonal linear polarizations were recorded and combined to Stokes $I$ in the gridding step (see below). Calibration against the continuum radio sources Hydra $\mathrm{A}$ and PKS B1934-638 was usually monitored on a daily or weekly basis to check system performance. However, all calibration was referenced to a continuously firing noise diode inserted in each of the 13 feeds at $45^{\circ}$ to the transducers. No evidence was found for any time variability in the noise diode amplitude. Neither is there any measurable gain-elevation effect at the Parkes telescope at these frequencies.

\subsection{Data Reduction through to Cubes}

The spectral data were reduced at the telescope in real time using the LiveDATA package. ${ }^{10}$ LiveData buffered the incoming data and applied position interpolation so that the correct beam position was assigned to the midpoint of each correlator spectrum. It then applied a barycentric correction using a fast Fourier transform shift and smoothed the data with a Tukey 25\% filter (see Barnes et al. 2001). Bandpass correction was then applied using reference spectra derived from the median of all spectra taken within 2 minutes $\left( \pm 2^{\circ}\right.$ in the scan direction) of each spectrum being corrected, except where a scan boundary was encountered. The reference spectrum was calculated separately for each beam and polarization. Up to 49 spectra, including the spectrum being corrected, were used to form each reference spectrum. The median statistic helped to suppress any RFI and any compact $\mathrm{H}_{\mathrm{I}}$ emission in the reference spectrum, which would otherwise appear as a spatial sidelobe. The bandpass was then corrected and the flux density calibration applied.

Automated and manual quality control measures were used to ensure that high data quality was maintained. Scans containing bad data, or scans containing telescope or correlator errors, were always reobserved, unless the errors were not recognized until after the completion of all ZOA observations.

Gridding into sky cubes was performed using GRIDZILLA, also written especially for the large data sets arising from multibeam observations (this data set consists of over 24 million spectra). Cubes were made using a simple top-hat median filter of all the data for a given spectral channel lying within a radius of $6^{\prime}$ of each pixel. Although this technique loses signal-to-noise ratio $(\mathrm{S} / \mathrm{N})$ relative to normal least-squares parametric techniques, it proved extremely effective in removing residual RFI or variable baseline ripple without extensive manual intervention.

\footnotetext{
${ }^{10}$ http://www.atnf.csiro.au/computing/software/livedata
}

As noted in Barnes et al. (2001), this particular gridding kernel requires an adjustment to the flux scale of $28 \%$ in order that the flux density of compact sources is preserved. This is applied directly to the cubes.

The pixel size of the final cubes is $4^{\prime}$ in R.A. and decl. and $13.2 \mathrm{~km} \mathrm{~s}^{-1}$ in velocity (at zero redshift). The gridded FWHP beam size is approximately 15,5 , compared with the normal Parkes beam at $1400 \mathrm{MHz}$ of $14 ! 3$, averaged across the 13 beams. Most of the considerable continuum emission was removed using the bandpass correction procedure described above. However, residual continuum emission remained, and this was further suppressed using the "scaled template method" (LUTHER) described by Barnes et al. (2001), where a weighted spectral template derived from the strongest sources in the field was scaled in amplitude to fit to the spectrum at each point in the cube. Finally, the data cubes were Hanning-smoothed to minimize ripple from strong Galactic $\mathrm{H}_{\mathrm{I}}$ signals. This results in a final velocity resolution of $27 \mathrm{~km} \mathrm{~s}^{-1}$. Spectra from the final cubes are available for download. ${ }^{11}$

\section{SAMPLE COMPILATION AND H I PARAMETERIZATION}

The initial HIPASS automated source list (Meyer et al. 2004) required the visual inspection of 33 times the number of galaxies that appeared in the final catalog. Owing to residual baseline excursions, especially in the presence of strong continuum, the efficiency of automated algorithms applied to the HIZOA data was even lower. Therefore, we chose to create the HIZOA catalog by visual searches alone. Each Hanningsmoothed cube was independently visually inspected by two or three authors, over the entire velocity range from -1200 to $12,700 \mathrm{~km} \mathrm{~s}^{-1}$, using the visualization package KARMA (Gooch 1996). The candidate lists created by each searcher were given to a single author who served as "adjudicator" for all cubes to produce as uniform a final catalog as possible. There were no quantitative a priori selection criteria, although for a signal to be accepted as extragalactic $\mathrm{HI}_{\mathrm{I}}$ it had to be at or exceeding the $5 \sigma$ level in peak flux density, at least marginally extended in velocity, and cleanly separated from Galactic $\mathrm{H}_{\mathrm{i}}$ in velocity space. Galactic gas tends to be more diffuse than external galaxies at our resolution, which are typically unresolved by the Parkes beam, although compact clouds certainly exist. While it is difficult to securely discriminate between high-velocity clouds and nearby dwarf galaxies in $\mathrm{H}$, the former are generally spatially more extended and visibly related to lower-velocity Galactic gas in our cubes. Generally, the H I sources showed distributions in velocity space consistent with known $\mathrm{H}_{\mathrm{I}}$ sources, i.e., either two-horned, flat-topped, or Gaussian profiles, and are well separated from Galactic gas velocities.

Once the final list of sources was made, the determination of $\mathrm{H}$ I parameters was done using the program MBSPECT within the MIRIAD package (Sault et al. 1995). For each source, zerothmoment maps were made, and the centroid of the $\mathrm{H}$ i emission was obtained by Gaussian fitting with either an FWHM equal to the gridded telescope beam for unresolved galaxies or a Gaussian of matching width in the case of resolved galaxies. Using this fitted position, the weighted sum of the emission along the spectral dimension of the data cube was calculated,

\footnotetext{
11 http://www.atnf.csiro.au/research/multibeam/release and select "Data Source = ZOA."
} 
producing the one-dimensional $\mathrm{H}$ I spectrum. Each spectrum was visually inspected, and a low-order polynomial was fitted to the line-free channels and subtracted, to remove any slowly varying spectral baseline. The total flux due to $\mathrm{H}_{\mathrm{I}}$ was then determined from this baseline-subtracted spectrum by integrating across the channels containing $21 \mathrm{~cm}$ emission. The heliocentric velocity (in the optical convention, $v=c z$ ) of each source was determined by taking the average of the velocity values at the $50 \%$ of peak flux points on the profile. Line widths at the $50 \%$ or $20 \%$ of peak flux levels were measured ( $w_{50}$ and $w_{20}$, respectively) using a width-maximizing algorithm. To correct for instrumental broadening due to the coarse velocity resolution after Hanning smoothing $\left(27 \mathrm{~km} \mathrm{~s}^{-1}\right)$, the values of $w_{50}$ and $w_{20}$ were decreased by 14 and $21 \mathrm{~km} \mathrm{~s}^{-1}$, respectively (Henning et al. 2000). The errors on all values were calculated using the formalism of Koribalski et al. (2004); they do not take into account baseline fitting errors. The errors that depend on line width (errors on heliocentric velocity, $w_{50}$ and $w_{20}$ ) were calculated using the observed (uncorrected) line widths and are therefore somewhat conservative values. In the case that the line width at $20 \%$ of peak flux was not robustly measurable owing to the signal's being too close to the noise level, no value is listed in the catalog (Table 2). Because the errors on heliocentric velocity and $w_{50}$ also depend on $w_{20}$, via measurement of the steepness of the profile edges, the sample average value of $\left(w_{20}-w_{50}\right)=42 \mathrm{~km} \mathrm{~s}^{-1}$ was used to calculate these two errors when $w_{20}$ was not available for a particular source.

\subsection{The Catalog}

The H I profiles for the first 32 of the 883 galaxies in the survey are shown in Figure 1 (the remainder are available in the online version of the journal). Vertical lines indicate the spectral ranges used for baseline subtraction, and the linear or polynomial fit is shown. On each profile, the peak, $50 \%$, and $20 \%$ levels are noted with dots. Table 2 is an example page of the full catalog (the table is published in its entirety in the electronic edition of the Astronomical Journal); it lists $\mathrm{H}_{\mathrm{I}}$ parameters and derived quantities for the galaxies in the following columns:

Columns (1) and (2)-Source name and flag. HIZOA galaxies in the Great Attractor region that were first reported by Juraszek et al. (2000) and in HIZOA-N (Donley et al. 2005) retain their original names, even if the position measurement has been improved by this survey. Names affected by those positional changes are indicated with an asterisk in Column (2). The H i parameters quoted are for the current work.

Columns (3a and 3b)-Equatorial coordinates (J2000.0) of the fitted position.

Columns ( $4 a$ and $4 b)$ _Galactic coordinates.

Column (5) - Reddening $E(B-V)$ as derived from the IRAS/ DIRBE maps (Schlegel et al. 1998) and corrected with a factor of 0.86 as derived by Schlafly \& Finkbeiner (2011).

Column (6)-Heliocentric velocity and error.

Column (7) — Velocity width at $50 \%$ of peak flux density, corrected for instrumental broadening, and associated error.

Column (8) - Velocity width at $20 \%$ of peak flux density, corrected for instrumental broadening, and associated error.

Column (9)—H I flux integral and associated error.
Column (10)_-Velocity of the galaxy corrected to the Local Group frame of reference via

$$
v_{\mathrm{LG}}=v_{\text {hel }}+300 \sin \ell \cos b
$$

Column (11) - Distance to the galaxy in Mpc, based on $v_{\mathrm{LG}}$ and $H_{0}=75 \mathrm{~km} \mathrm{~s}^{-1} \mathrm{Mpc}^{-1}$.

Column (12)_Logarithm of the H I mass.

\section{H I PROPERTIES OF THE SAMPLE}

An overview of the Hi properties of the sample of 883 galaxies is shown in Figure 2. The top panel shows the distribution of the galaxies' recessional velocities in the Local Group frame of reference. The distribution generally reflects the noise-limited sensitivity of the survey, but also reveals the overdensity of galaxies in the Great Attractor region, at about $5000 \mathrm{~km} \mathrm{~s}^{-1}$. This feature is apparent despite the averaging across a variety of large-scale structures in this wide-angle survey, described in more detail in Section 7. The HIZOA survey was designed to map large-scale structure at the distance of the Great Attractor more completely than the HIPASS survey, which did not have the sensitivity to trace structure beyond about $4000 \mathrm{~km} \mathrm{~s}^{-1}$ (the rms for HIPASS is $13-22 \mathrm{mJy}$ in the Galactic plane versus an average of $6 \mathrm{mJy}$ for HIZOA). This allowed HIZOA to detect galaxies at lower $\mathrm{H}$ i masses and thus probe lower down the $\mathrm{H}$ i mass function at Great Attractor distances (e.g., Figure 3).

The next panel shows the distribution of $\mathrm{H}_{\mathrm{I}}$ line widths measured at half-peak, $w_{50}$, which has a mean value of $163 \mathrm{~km} \mathrm{~s}^{-1}$ and a median of $147 \mathrm{~km} \mathrm{~s}^{-1}$, with large, nonGaussian variation from the smallest value of $17 \mathrm{~km} \mathrm{~s}^{-1}$ to $699 \mathrm{~km} \mathrm{~s}^{-1}$ maximum. Three detections, which we believe are not confused, have $w_{50}>600 \mathrm{~km} \mathrm{~s}^{-1}$, the largest of which $\left(\mathrm{J} 1416-58, w_{50}=699 \mathrm{~km} \mathrm{~s}^{-1}\right)$ is a perfectly edge-on spiral. The iteratively clipped rms noise at the location of each detected source is shown in the next panel. Owing to the clipping and the limited velocity range over which the measurement is made, this is normally much lower than the overall cube rms of $6 \mathrm{mJy}$. Some galaxies were found in areas with rms as high as $10-20 \mathrm{mJy}$ at the edge of the field or near strong Galactic foreground radiation. The lowest panel shows the distribution of the $\mathrm{H}$ I masses, which ranges from 6.8 to 10.8 in the logarithm, with a mean of 9.5 and a median of 9.6. The most massive H i object, HIZOA J0836-43, has been the subject of radio interferometry and deep NIR follow-up observations, showing it to be a high surface brightness, massive disk galaxy with an extended $\mathrm{H}_{\mathrm{I}}$ disk (Donley et al. 2006; Cluver et al. 2008, 2010).

The well-known Circinus galaxy lies within the survey area and is redetected here as HIZOA J1413-65. The narrow velocity width, low $\mathrm{H}_{\mathrm{I}}$ mass $\left(\log M_{\mathrm{HI}}=7.24\right)$ detection HIZOA J1353-58, less than $8^{\circ}$ from Circinus, has a recessional velocity that is only about $200 \mathrm{~km} \mathrm{~s}^{-1}$ different from the large galaxy. At the low recession velocities of these objects of only a few hundred kilometers per second, Hubble flow distances are very uncertain, but it seems likely that this newly detected object is a previously unknown galaxy that is related to the Circinus galaxy. As its velocity corrected to the Local Group frame is negative, and thus a Hubble distance is 
Table 2

$\mathrm{H}_{\mathrm{I}}$ and Derived Parameters

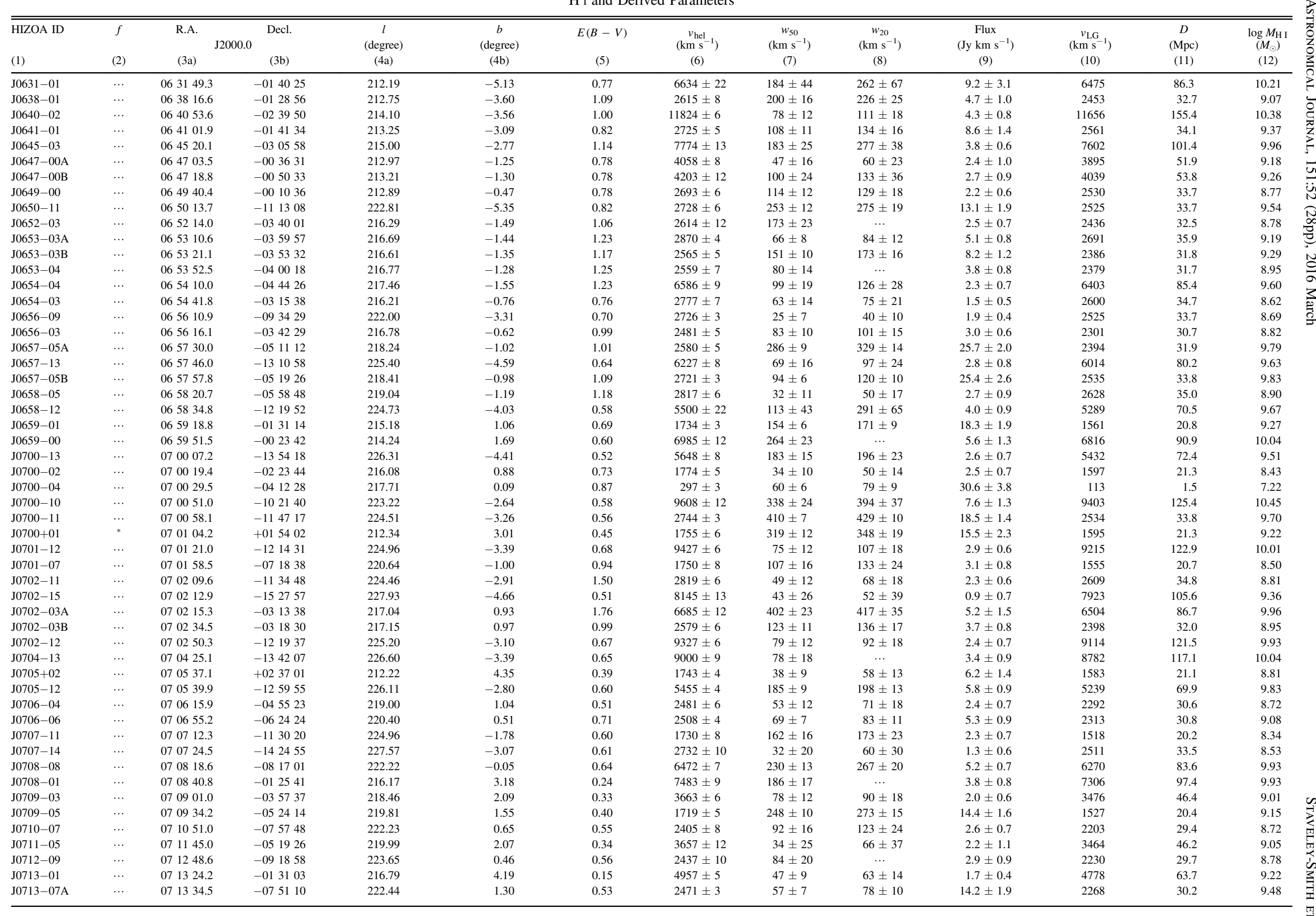

(This table is available in its entirety in machine-readable and Virtual Observatory (VO) forms.) 


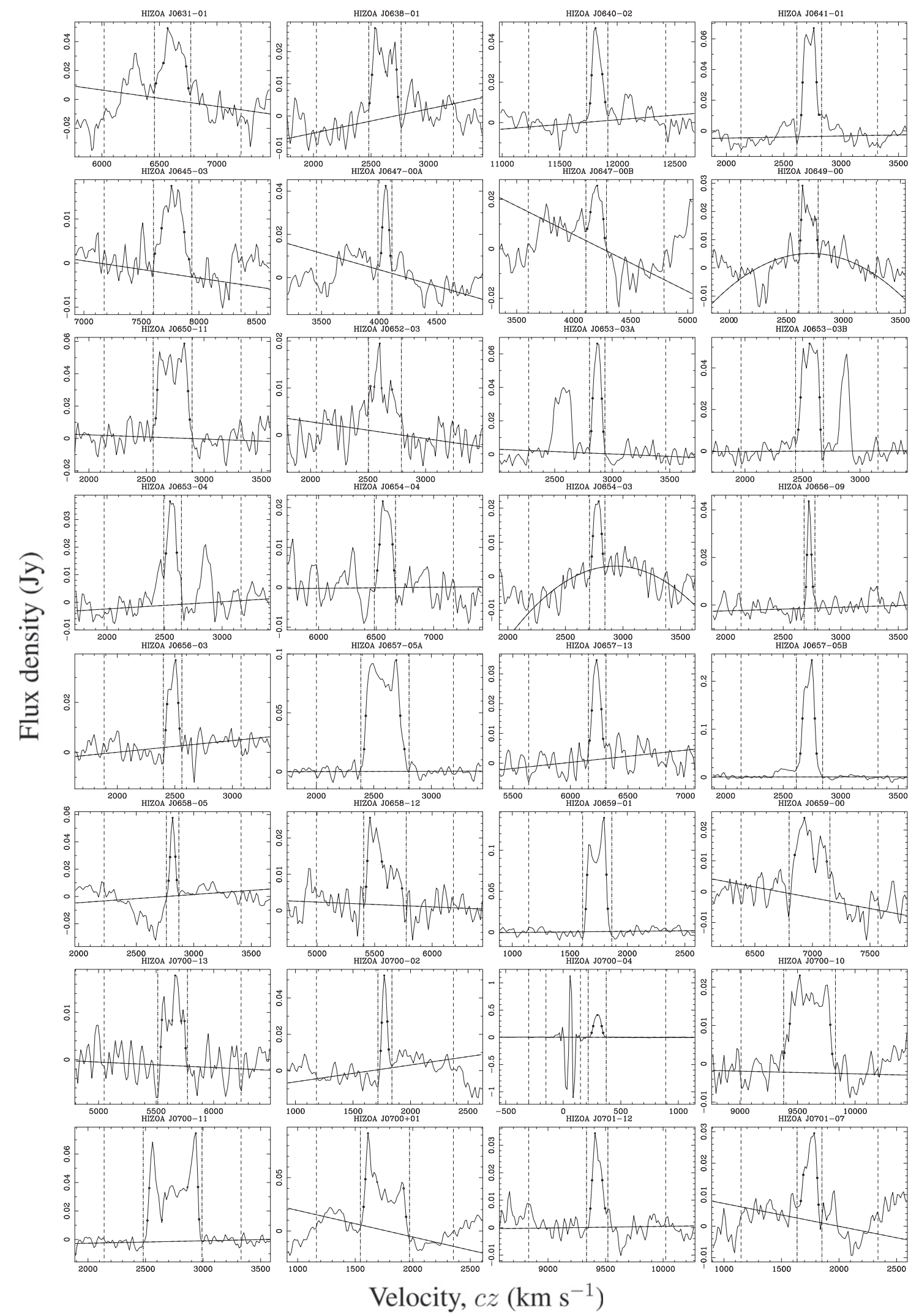

Figure 1. Example H I spectra of the newly detected galaxies in the HIZOA-S survey. Low-order baselines (indicated by the solid line) are fitted, excluding the detections themselves (which are bracketed by the dot-dashed vertical lines) and excluding the low- and high-velocity edges to the left and right of the dashed vertical lines, respectively. The $20 \%$ and $50 \%$ profile markers are visible. All the spectra are available in the figure set.

(The complete figure set (28 images) is available.) 

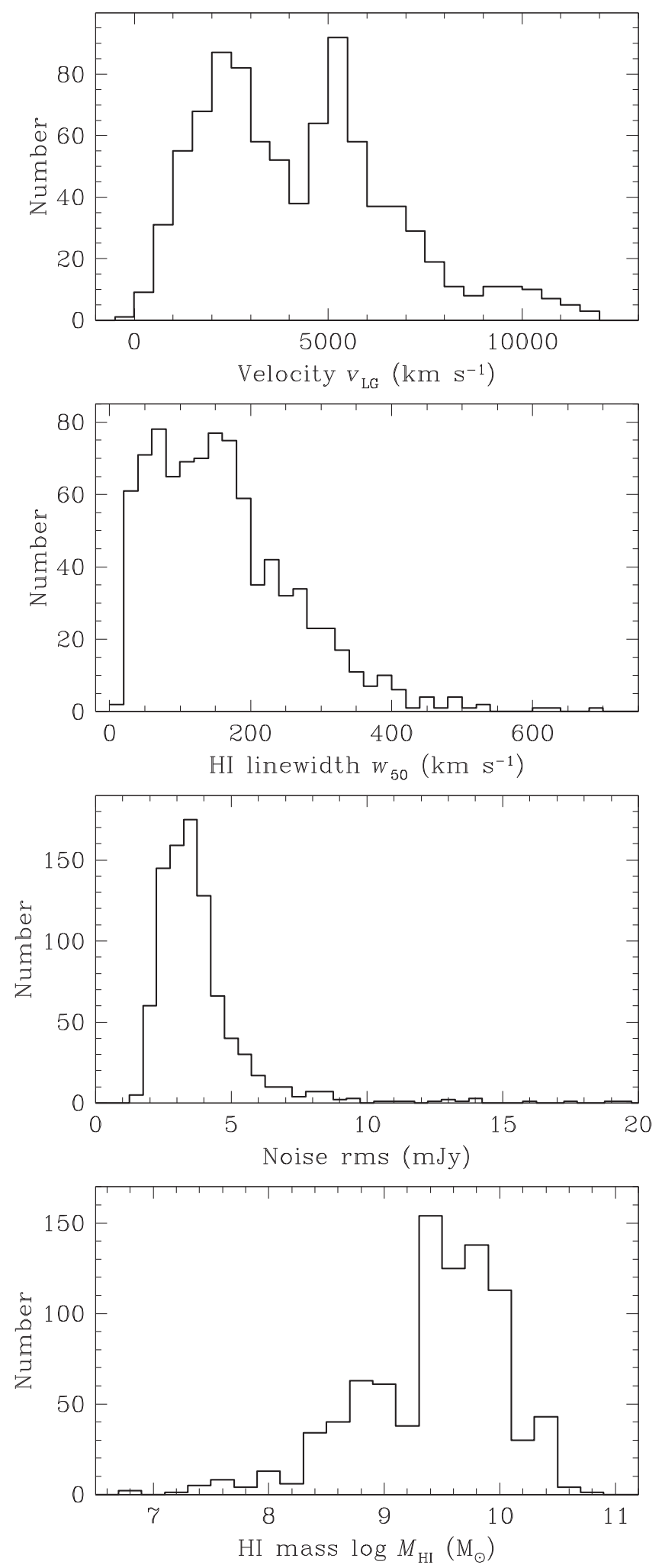

Figure 2. H I parameters of the 883 galaxies detected in the HIZOA-S survey. From top to bottom the histograms display the radial velocity $v_{\mathrm{LG}}$, the line width $w_{50}$, the clipped rms noise at the position of the detected galaxy, and the $\mathrm{H}$ I mass distribution.

undefined in this frame, we adopt a redshift-independent distance measurement to Circinus, 4.2 Mpc (Tully et al. 2009), as the distance to both HIZOA J1413-65 and HIZOA J1353-58.

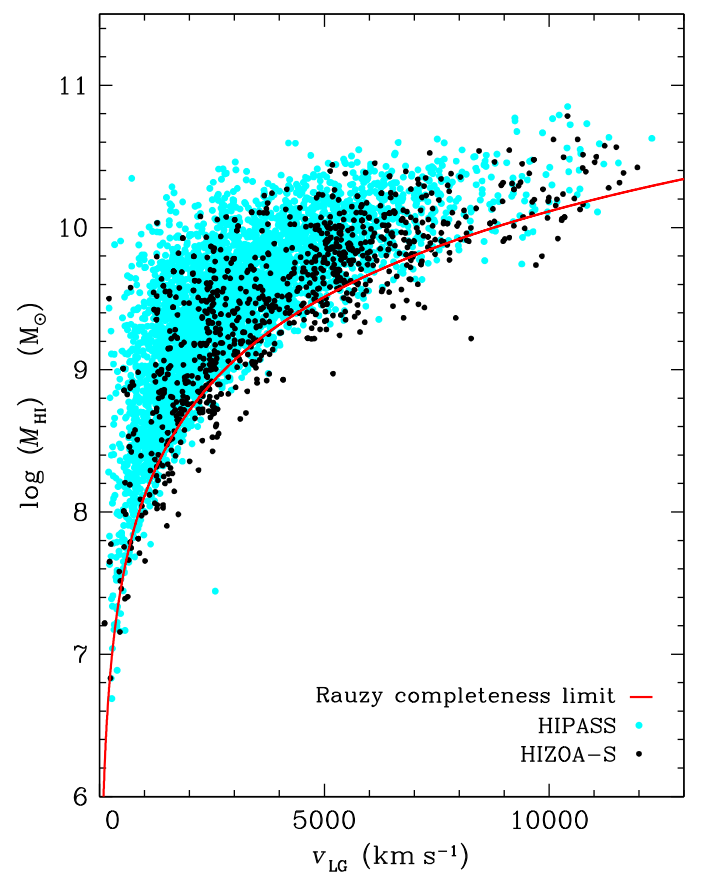

Figure 3. H I mass vs. velocity in the Local Group frame for the HIZOA-S (black) and HIPASS samples (light blue; Meyer et al. 2004). The Rauzy completeness limit for the flux integral alone $\left(3.1 \mathrm{Jy} \mathrm{km} \mathrm{s}^{-1}\right)$ is shown as a red curve.

The Circinus galaxy and four other detections (J1509-52, J1514-52, J1532-56, J1616-55) are significantly extended, that is, resolved with respect to the 15.5 beam.

Figure 3 shows the distribution of $\mathrm{H}$ I mass as a function of velocity in the Local Group frame for the HIZOA-S and HIPASS (Meyer et al. 2004) samples. This illustrates the higher sensitivity of the HIZOA survey over HIPASS, as HIZOA finds lower $\mathrm{H}_{\text {I }}$ mass galaxies at all distances, but also reflects the smaller area covered, which produced fewer detections overall, compared to HIPASS. The completeness limit for HIZOA-S is also shown in the figure and is fully described in Section 6. As in the top panel of Figure 2, despite the inclusion of a variety of large-scale structures probed in this wide-angle survey, clear overdensities at $\sim 2500 \mathrm{~km} \mathrm{~s}^{-1}$ (Hy/Ant and Puppis) and $\sim 5000 \mathrm{~km} \mathrm{~s}^{-1}$ (the Great Attractor region) are evident in the velocity distribution of the HIZOA galaxies. These structures are discussed in detail in Section 7.

\section{MULTIWAVELENGTH COUNTERPARTS}

To find counterparts for the $\mathrm{H}_{\mathrm{I}}$ detections, we have (a) searched the literature through online databases and (b) searched images at various wavelengths.

Our main literature search was done using the NASA/IPAC Extragalactic Database (NED). ${ }^{12}$ We submitted a list of the $\mathrm{H}_{\mathrm{I}}$ detections through the NED batch service using a 4.5 search radius (status 2013 August). For comparison, we also conducted a search with the $\operatorname{SIMBAD}^{13}$ Astronomical Database. There were only a couple of cases in which SIMBAD had a galaxy listed but NED did not. On the other hand, SIMBAD listed more (unclassified) IRAS sources than NED, which we looked at (using their colors as a first-order

\footnotetext{
12 http://ned.ipac.caltech.edu/

${ }^{13}$ http://simbad.u-strasbg.fr/simbad/
} 
indicator for galaxies). The searches also supplied a redshift where available. Particular attention was paid to galaxies with optical redshifts since these have negligible positional uncertainties.

For the visual search, we downloaded images from the SuperCOSMOS Sky Surveys ${ }^{14}$ ( $B$ band), the Digitized Sky Surveys (DSS $)^{15}$ ( $R$ and $I$ band), 2 MASS $^{16}$ ( $K_{s}$ band), as well as UKIDSS $^{17}$ and VISTA ${ }^{18}$ wherever possible (mainly $K_{s}$ band, but if not available, we used the band closest in wavelength). Finally, we added $K_{s}$-band images (as well as in $J$ or $H$ when necessary) that were obtained at the Infrared Survey Facility at Sutherland (IRSF) for the purpose of extracting photometry on all galaxies within the HIZOA search circles. A first set of this catalog was published by Williams et al. (2014), and the rest is in preparation (Said et al. 2015).

While the field of view of the IRSF images is $8^{\prime} \times 8^{\prime}$, the downloaded images were extracted as $9^{\prime} \times 9^{\prime}$ or, in some cases, $10^{\prime} \times 10^{\prime}$. The 2 MASS images are only 8.5 wide, and clearly not all of the 4 .5-radius search circle could be covered in a single image. 2MASS images covering the missing bits of the search area were added when no suitable candidate could be found at any other passband. In addition, the DIRBE/IRAS maps ${ }^{19}$ (Schlegel et al. 1998) were downloaded and viewed together with the images to take into account the amount and distribution of extinction in the area. WISE ${ }^{20}$ images (Wright et al. 2010) were used to better distinguish between galaxies and Galactic objects.

Images were viewed with $\mathrm{DS}^{21}$ and overlaid with regions indicating (i) the $\mathrm{H}$ I position, (ii) the 4.5 search radius, and (iii) the positions of the objects found with NED and SIMBAD. We added positions from HIPASS (Meyer et al. 2004) and the HIZSS shallow survey catalog (Henning et al. 2000). While displaying the Hiparameters, these images were searched simultaneously by eye to detect any possible galaxy in the field. Most galaxies found in the literature were visible in at least one passband, and quite often unpublished galaxies could be found, especially blue late-type galaxies (often not or barely visible in the NIR) or, on deep NIR images, galaxies at high extinctions or near the Galactic bulge, where 2MASS has difficulty detecting galaxies owing to severe star crowding.

If more than one galaxy was found, the most likely counterpart was selected based on the $\mathrm{HI}$ parameters, the appearance of the galaxy on the images, and the extinction information. In some cases no reasonable decision could be made on which of the galaxies is the counterpart, and hence both possible (but ambiguous) candidates are retained. In other cases, the chosen candidate was classified as "probable," indicating that another galaxy could also be considered as the counterpart, albeit less likely. This class was also used when no second candidate was visible but the appearance of the galaxy was not an ideal match with the $\mathrm{H}$ i parameters. Finally, sometimes more than one candidate is considered to contribute to the profile (i.e., the profile is likely to be "confused").

\footnotetext{
${ }^{14}$ http://www-wfau.roe.ac.uk/sss/

15 http://www3.cadc-ccda.hia-iha.nrc-cnrc.gc.ca/en/dss/

$16 \mathrm{http}: / /$ irsa.ipac.caltech.edu/applications/2MASS/

${ }^{17} \mathrm{http}: / /$ surveys.roe.ac.uk/wsa/

18 http://horus.roe.ac.uk/vsa/

19 http://www.astro.princeton.edu/ schlegel/dust/

${ }^{20} \mathrm{http}: / /$ irsa.ipac.caltech.edu/applications/wise/

${ }^{21}$ http://ds9.si.edu/
}

Some of the counterparts lie beyond the nominal 4.5 search radius. They were found when a larger search circle seemed appropriate (see the discussion below).

The counterparts are listed in Table 3 (an example page of which is shown here). It is divided into three parts, reflecting the different types or counterparts found as explained above. Table 3(a) lists those HIZOA detections that have either a single or no counterpart. Table 3(b) presents detections where more than one galaxy is assumed to contribute to the $\mathrm{H}$ i profile (note that where the confusing partner was another of our H Idetections we do not list them separately). Finally, Table 3(c) lists those cases where more than one candidate was found but, judged by the profile, only one of them is the likely counterpart. The columns are as follows.

Column (1): source name as in Table 2.

Columns (2a) and (2b): Galactic coordinates of the $\mathrm{HI}$ detection.

Column (3): distance to the H I galaxy in Mpc, as in Table 2.

Column (4): logarithm of the H I mass, as in Table 2.

Column (5): extinction in the $B$ band, converted from $E(B-V)$ given in Table 2 using $R_{\mathrm{B}}=4.14$. A star denotes an extinction value deemed to be uncertain during the search (e.g., owing to high spatial variability).

Column (6): classification of the counterpart; " $\mathrm{d}$ " = definite, "p" = probable, "a" = ambiguous, "c" = confused candidate, "-" = no candidate.

Column (7): flags for counterparts in major catalogs: "I" stands for IRAS Point Source Catalog (Helou \& Walker 1988), "M" for 2MASX (Jarrett et al. 2000), "W" for Williams et al. 2014 (the IRSF catalog), "H" for the HIPASS catalogs (south, Meyer et al. 2004; north, Wong et al. 2006) or "h" for other HIPASS publications (Kilborn et al. 2002; Ryan-Weber et al. 2002), "S" for the H I Parkes ZOA Shallow Survey (HIZSS; Henning et al. 2000), and "Z" for earlier HIZOA publications (Juraszek et al. 2000; Donley et al. 2005).

Column (8): velocity in the literature (see NED and HyperLEDA $^{22}$ ): "o" = optical (and, in one case, " $\mathrm{x}$ " for $\mathrm{X}$-ray), "h" = H I (note that Juraszek et al. [2000] and Henning et al. [2000] are not included here since their $\mathrm{H}$ I parameters are not independent of ours, but see flags in Col. [7]).

Column (9): source "l" for name and coordinates: $\mathrm{N}$ is listed in NED, $\mathrm{S}$ is listed in SIMBAD, $\mathrm{c}$ is coordinates measured on DSS or NIR images (note that some published coordinates were not centered properly, so we give the measured ones).

Column (10): note in the Appendix.

Columns (11a) and (11b): equatorial coordinates (J2000.0) of the counterpart.

Column (12): distance between the $\mathrm{H}$ I fitted position and the counterpart position in arcminutes.

Column (13): one name in the literature in this order of preference: NGC, ESO, RKK/WKK, CGMW, 2MASS, others.

\subsection{Results of Counterpart Search}

Of the $883 \mathrm{H}$ i detections, we found cross-matches for 688 (78\%). At least $18(2 \%) \mathrm{H}$ I detections have more than one counterpart, that is, more than one galaxy contributes to the $\mathrm{H}$ I profile; see Table 3(b). Not counted here are detections that are confused but could be separated into their H I components, which are all listed in Table 2. For another $17(2 \%) \mathrm{HI}$ detections, there is no unambiguous counterpart. Instead, we

\footnotetext{
22 http://leda.univ-lyon1.fr/
} 
Table 3

Cross-matches of the $\mathrm{H}$ I Detections

\begin{tabular}{|c|c|c|c|c|c|c|c|c|c|c|c|c|c|c|}
\hline $\begin{array}{l}\text { HIZOA ID } \\
\text { (1) }\end{array}$ & $\begin{array}{c}l \\
\text { (degree) } \\
(2 \mathrm{a})\end{array}$ & $\begin{array}{c}b \\
\text { (degree) } \\
\text { (2b) }\end{array}$ & $\begin{array}{c}D \\
\text { (Mpc) } \\
\text { (3) }\end{array}$ & $\begin{array}{c}\log M_{\mathrm{HI}} \\
\left(M_{\odot}\right) \\
(4)\end{array}$ & $\begin{array}{c}A_{\mathrm{B}} \\
(\mathrm{mag}) \\
(5)\end{array}$ & $\begin{array}{l}\text { Class } \\
\text { (6) }\end{array}$ & $\begin{array}{c}\text { IMWHSZ } \\
\text { (7) }\end{array}$ & (8) & (9) & Note & $\begin{array}{l}\text { R.A. } \\
\text { (11a) }\end{array}$ & $\begin{array}{ll}\text { J2000.0 } & \text { Decl. } \\
& (1 \mathrm{~b})\end{array}$ & $\begin{array}{l}d_{\text {sep }} \\
\text { (12) }\end{array}$ & $\begin{array}{l}\text { Name } \\
(') \\
(13)\end{array}$ \\
\hline \multicolumn{15}{|c|}{ (a) $\mathrm{H}$ I detections with single cross-matches: } \\
\hline J0631-01 & 212.19 & -5.13 & 86.3 & 10.21 & 3.2 & $\mathrm{p}$ & $---\mathrm{H}--$ & $-\mathrm{h}$ & $-c$ & $\mathrm{n}$ & 063209.5 & -013653 & 6.1 & \\
\hline J0638-01 & 212.75 & -3.60 & 32.7 & 9.07 & 4.5 & d & $-\cdots-$. & -- & $-c$ & - & 063820.3 & -012838 & 1.0 & $\ldots$ \\
\hline J0640-02 & 214.10 & -3.56 & 155.4 & 10.38 & 4.2 & $\mathrm{~d}$ & -M- - - - & -- & N- & - & 064056.02 & -023832.6 & 1.4 & 2MASX J06405601-0238326 \\
\hline J0641-01 & 213.25 & -3.09 & 34.1 & 9.37 & 3.4 & d & $-\ldots--$ & -- & $-c$ & - & 064058.4 & -014403 & 2.6 & \\
\hline J0645-03 & 215.00 & -2.77 & 101.4 & 9.96 & 4.7 & d & -M- - - - & -- & N- & - & 064523.44 & -030714.3 & 1.5 & 2MASX J06452346-0307141 \\
\hline J0647-00A & 212.97 & -1.25 & 51.9 & 9.18 & 3.2 & - & $-\ldots--$ & -- & & $\mathrm{n}$ & $\ldots$ & $\ldots$ & & $\ldots$ \\
\hline J0647-00B & 213.21 & -1.30 & 53.8 & 9.26 & 3.2 & $\mathrm{p}$ & -M- - - - & -- & N- & - & 064713.16 & -004950.1 & 1.6 & 2MASX J06471318-0049501 \\
\hline J0649-00 & 212.89 & -0.47 & 33.7 & 8.77 & 3.2 & d & $-\cdots$ & -- & $-c$ & - & 064929.7 & -000903 & 3.1 & $\ldots$ \\
\hline J0650-11 & 222.81 & -5.35 & 33.7 & 9.54 & 3.4 & $\mathrm{~d}$ & IM-H- - & oh & N- & - & 065010.61 & -111513.0 & 2.2 & CGMW 1-0411 \\
\hline $\mathrm{J} 0652-03$ & 216.29 & -1.49 & 32.5 & 8.78 & 4.4 & d & -M- - - - & -- & N- & - & 065200.20 & -034034.2 & 3.5 & CGMW 1-0424 \\
\hline J0653-03A & 216.69 & -1.44 & 35.9 & 9.19 & 5.1 & $\mathrm{p}$ & - - -H- - & $-\mathrm{h}$ & $-c$ & $\mathrm{n}$ & 065313.6 & -040004 & 0.7 & $\ldots$ \\
\hline J0653-03B & 216.61 & -1.35 & 31.8 & 9.29 & 4.8 & d & - - -H- - & $-\mathrm{h}$ & $-c$ & $\mathrm{n}$ & 065321.8 & -035257 & 0.6 & $\ldots$ \\
\hline J0653-04 & 216.77 & -1.28 & 31.7 & 8.95 & 5.2 & - & $-\ldots-$ & -- & & $\mathrm{n}$ & $\ldots$ & $\ldots$ & $\ldots$ & $\ldots$ \\
\hline J0654-04 & 217.46 & -1.55 & 85.4 & 9.60 & 5.1 & d & ----- & -- & $-c$ & - & 065359.7 & -044216 & 3.3 & $\ldots$ \\
\hline J0654-03 & 216.21 & -0.76 & 34.7 & 8.62 & 3.2 & $\mathrm{p}$ & ---- & -- & $-c$ & - & 065439.0 & -031623 & 1.0 & \\
\hline J0656-09 & 222.00 & -3.31 & 33.7 & 8.69 & 2.9 & d & ---- & -- & $-c$ & - & 065609.2 & -093631 & 2.1 & \\
\hline J0656-03 & 216.78 & -0.62 & 30.7 & 8.82 & 4.1 & $\mathrm{p}$ & - - -H- - & $-\mathrm{h}$ & $-c$ & - & 065623.0 & -034526 & 3.4 & \\
\hline J0657-05A & 218.24 & -1.02 & 31.9 & 9.79 & 4.2 & d & IM-H- - & $-\mathrm{h}$ & N- & $\mathrm{n}$ & 065721.50 & -050859.6 & 3.1 & CGMW 1-0464 \\
\hline J0657-13 & 225.40 & -4.59 & 80.2 & 9.63 & 2.7 & d & -M- - - - & -. & N- & - & 065740.63 & -131115.2 & 1.3 & 2MASX J06574062-1311150 \\
\hline J0657-05B & 218.41 & -0.98 & 33.8 & 9.83 & 4.5 & $\mathrm{~d}$ & IMWHS- & oh & N- & $\mathrm{n}$ & 065802.90 & -052041.0 & 1.8 & CGMW 1-0470 \\
\hline J0658-05 & 219.04 & -1.19 & 35.0 & 8.90 & 4.9 & - & - - -H- - & $-\mathrm{h}$ & - & - & $\ldots$ & $\ldots$ & & \\
\hline J0658-12 & 224.73 & -4.03 & 70.5 & 9.67 & 2.4 & d & -M- - - - & -- & N- & - & 065830.63 & -122200.8 & 2.4 & CGMW 1-0472 \\
\hline J0659-01 & 215.18 & 1.06 & 20.8 & 9.27 & 2.8 & d & - - -HS- & $-\mathrm{h}$ & NC & - & 065920.4 & -013131 & 0.5 & CGMW 1-0476 \\
\hline J0659-00 & 214.24 & 1.69 & 90.9 & 10.04 & 2.5 & $\mathrm{~d}$ & IM- - - - & o- & N- & - & 065953.72 & -002527.5 & 1.8 & CGMW 1-0479 \\
\hline J0700-13 & 226.31 & -4.41 & 72.4 & 9.51 & 2.2 & $\mathrm{~d}$ & - -W- - - & - - & $-c$ & - & 070002.4 & -135205 & 2.5 & $\ldots$ \\
\hline J0700-02 & 216.08 & 0.88 & 21.3 & 8.43 & 3.0 & $\mathrm{~d}$ & $---\mathrm{H}--$ & $-\mathrm{h}$ & $-c$ & - & 070031.8 & -022245 & 3.3 & $\ldots$ \\
\hline J0700-10 & 223.22 & -2.64 & 125.4 & 10.45 & 2.4 & $\mathrm{p}$ & -M- - - - & -- & N- & - & 070034.38 & -102015.1 & 4.3 & 2MASX J07003437-1020151 \\
\hline J0700-11 & 224.51 & -3.26 & 33.8 & 9.70 & 2.3 & d & -M-H- - & $-\mathrm{h}$ & N- & - & 070056.14 & -114734.3 & 0.6 & CGMW 1-0488 \\
\hline $\mathrm{J} 0700+01$ & 212.34 & 3.01 & 21.3 & 9.22 & 1.8 & $\mathrm{~d}$ & IM-H-Z & oh & N- & $\ldots$ & 070103.30 & -015440.6 & 0.7 & UGC03630 \\
\hline J0701-12 & 224.96 & -3.39 & 122.9 & 10.01 & 2.8 & d & -M- - - - & $-\mathrm{h}$ & N- & - & 070125.75 & -121522.0 & 1.4 & CGMW 1-0491 \\
\hline J0701-07 & 220.64 & -1.00 & 20.7 & 8.50 & 3.9 & $\mathrm{~d}$ & $-\ldots--$ & -- & $-c$ & - & 070147.2 & -071936 & 3.0 & \\
\hline J0702-11 & 224.46 & -2.91 & 34.8 & 8.81 & 6.2 & - & ----- & -- & & -- & - & $\ldots$ & & \\
\hline J0702-15 & 227.93 & -4.66 & 105.6 & 9.36 & 2.1 & d & ----- & -- & $-c$ & - & 070218.1 & -152647 & 1.7 & $\ldots$ \\
\hline $\mathrm{J} 0702-03 \mathrm{~A}$ & 217.04 & 0.93 & 86.7 & 9.96 & $7.3^{*}$ & d & -M- - - - & -- & N- & - & $0702 \quad 15.33$ & -031346.6 & 0.1 & CGMW 1-0497 \\
\hline $\mathrm{J} 0702-03 \mathrm{~B}$ & 217.15 & 0.97 & 32.0 & 8.95 & 4.1 & - & $---\mathrm{H}--$ & $-\mathrm{h}$ & & - - & - & $\ldots$ & $\ldots$ & $\ldots$ \\
\hline J0702-12 & 225.20 & -3.10 & 121.5 & 9.93 & 2.8 & d & $-\ldots-$. & -- & $-c$ & - & 070252.9 & -122016 & 0.9 & $\ldots$ \\
\hline J0704-13 & 226.60 & -3.39 & 117.1 & 10.04 & 2.7 & $\mathrm{~d}$ & -M- - - - & -- & N- & $\mathrm{n}$ & 070425.31 & -134626.0 & 4.3 & CGMW 1-0523 \\
\hline J0705+02 & 212.22 & 4.35 & 21.1 & 8.81 & 1.6 & d & $---H-\mathrm{Z}$ & $-\mathrm{h}$ & $\mathrm{NC}$ & - & 070538.5 & -023718 & 0.4 & [H92] 16 \\
\hline J0705-12 & 226.11 & -2.80 & 69.9 & 9.83 & 2.5 & d & $---\mathrm{H}--$ & $-\mathrm{h}$ & $-c$ & - & 070541.8 & -130030 & 0.8 & $\ldots$ \\
\hline J0706-04 & 219.00 & 1.04 & 30.6 & 8.72 & 2.1 & d & ---- & -- & $-c$ & - & 070614.0 & -045709 & 1.8 & $\ldots$ \\
\hline J0706-06 & 220.40 & 0.51 & 30.8 & 9.08 & 2.9 & d & ----- & -- & $-c$ & - & 0706 58.0: & $-062510:$ & 1.0 & $\ldots$ \\
\hline J0707-11 & 224.96 & -1.78 & 20.2 & 8.34 & 2.5 & d & $-\mathrm{M}-\mathrm{-}_{-}-$ & -- & N- & - & 070716.68 & -113027.8 & 1.1 & 2MASX J07071668-1130281 \\
\hline J0707-14 & 227.57 & -3.07 & 33.5 & 8.53 & 2.5 & - & $-\cdots-$ & -- & & -- & - & .. & $\ldots$ & $\ldots$ \\
\hline J0708-08 & 222.22 & -0.05 & 83.6 & 9.93 & 2.7 & $\mathrm{~d}$ & - . . . . - & -- & $-c$ & - & 070825.8 & -081701 & 1.8 & $\ldots$ \\
\hline J0708-01 & 216.17 & 3.18 & 97.4 & 9.93 & 1.0 & d & $\cdots \cdots$ & -- & N- & - & 070836.83 & -012630.8 & 1.3 & 2MASX J07083681-0126306 \\
\hline J0709-03 & 218.46 & 2.09 & 46.4 & 9.01 & 1.4 & $\mathrm{~d}$ & $\ldots$ & -- & $\mathrm{Sc}$ & $\mathrm{n}$ & 070857.8 & -035839 & 1.3 & DSH J0708.9-0358 \\
\hline J0709-05 & 219.81 & 1.55 & 20.4 & 9.15 & 1.6 & d & IM-HS- & oh & $\mathrm{N}-$ & - & 070934.59 & -052540.5 & 1.4 & CGMW 1-0575 \\
\hline J0710-07 & 222.23 & 0.65 & 29.4 & 8.72 & 2.3 & $\mathrm{p}$ & 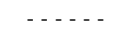 & -- & $-c$ & - & 071057.9 & -075431 & 3.7 & \\
\hline J0711-05 & 219.99 & 2.07 & 46.2 & 9.05 & 1.4 & d & $\ldots \ldots$ & -- & $-c$ & - & 071154.0 & -051813 & 2.5 & $\ldots$ \\
\hline J0712-09 & 223.65 & 0.46 & 29.7 & 8.78 & 2.3 & d & IMW- . - & $-\mathrm{h}$ & N- & - & 071248.61 & -091822.6 & 0.6 & CGMW 1-0617 \\
\hline
\end{tabular}

(This table is available in its entirety in machine-readable and Virtual Observatory (VO) forms.) 


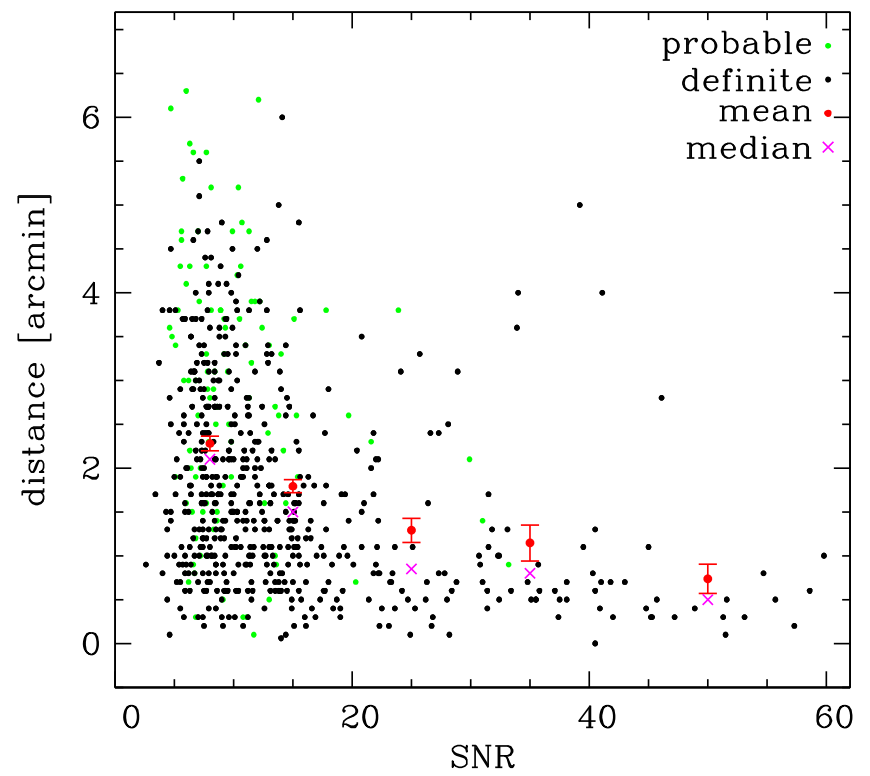

Figure 4. Separation between the $H_{\text {I }}$ position and the optical/NIR counterpart position as a function of $\mathrm{S} / \mathrm{N}$. The mean and error and the median of the definite candidates (black dots) are indicated with red dots and magenta crosses, respectively, for the $\mathrm{S} / \mathrm{N}$ bins 6-10, 10-20, 20-30, 30-40, and 40-60; not included are the probable candidates (green dots). The beam size is 15.5 , and the pixel size is $4{ }^{\prime} 0$.

list two possible candidates each in Table 3(c) (note that these profiles do not show any indication of confusion).

A total of $295(33 \%)$ detections have been detected previously in $\mathrm{HI}$, where $256(28 \%)$ have been detected with HIPASS and $110(12 \%)$ are also listed in the HIZSS catalog.

Of the 708 cross-matches found (from Table 3(c) we count arbitrarily only the first entry each), $328(46 \%)$ are listed in NED or SIMBAD $\left(N_{\mathrm{S}}=3\right)$, an additional $138(19 \%)$ are detected by Williams et al. (2014) on deep NIR images, and $240(34 \%)$ have no optical/IR counterparts. There are 128 (18\%) IRAS counterparts (with a further $8(1 \%)$ uncertain identifications), $248(35 \%)$ are listed in 2MASS, and $58(8 \%)$ have an optical (that is, independent) velocity.

Of the 558 single cross-matches (that is, matches from Table 3(a) only and excluding probable matches), the median distance between the $\mathrm{H}$ i position and the actual position is $1 ! 4$, while $95 \%$ of the cross-matches have a distance $<3$ ! 8 . The dependence of the mean and median distance on $\mathrm{S} / \mathrm{N}$ is shown in Figure 4. Aside from low $\mathrm{S} / \mathrm{N}$, unusually large distances are probably due to offset emission or confusion (two or more galaxies contributing to the emission) (see Table 3(b)).

For example, in Figure 4, two of the five objects with $\mathrm{S} /$ $\mathrm{N}>30$ and distance $>2^{\prime}$ have $|b|>5^{\circ}$ and two are extended. In one case (J0817-29B with $v=1654 \mathrm{~km} \mathrm{~s}^{-1}$, $w_{50}=158 \mathrm{~km} \mathrm{~s}^{-1}, \mathrm{~S} / \mathrm{N}=41$, dist $=4^{\prime}$ ) there is a very bright, nearby $\mathrm{HI}$ detection $20^{\prime}$ away (J0817-30 with $v=1662 \mathrm{~km} \mathrm{~s}^{-1}, w_{50}=186 \mathrm{~km} \mathrm{~s}^{-1}$ ).

Koribalski et al. (2004) find that $95 \%$ of their optical crossmatches have a positional offset $<3$ ! 2 . If we use a cut in peak flux similar to theirs $\left(S_{p}=0.116 \mathrm{Jy}\right)$, our offset for $95 \%$ of our cross-matches is $<3 ! 7$. Reasons for the difference can be our smaller sample size for this cut $(N=57)$ and the fact that Koribalski et al. use the nearest match in HyperLEDA, while we make an informed decision on which of the close-by

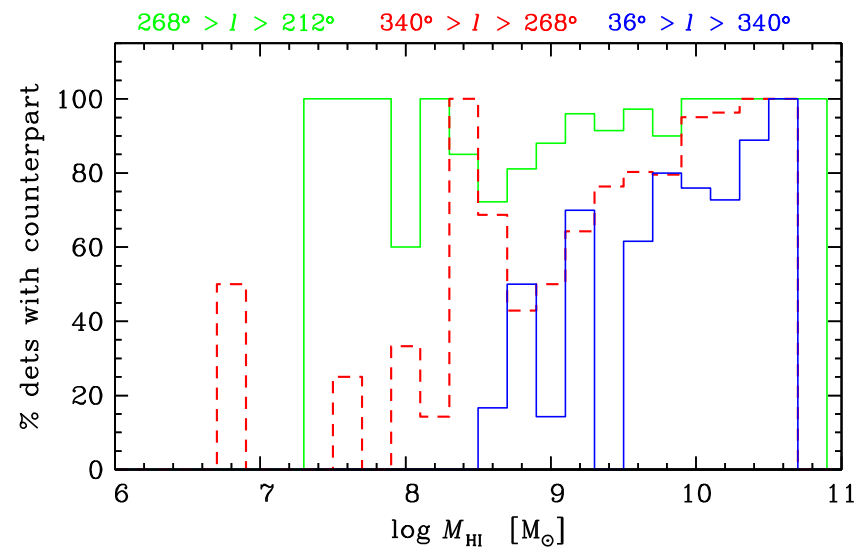

Figure 5. H i mass of HIZOA-S detections with counterparts identified, for different Galactic longitudes as indicated.

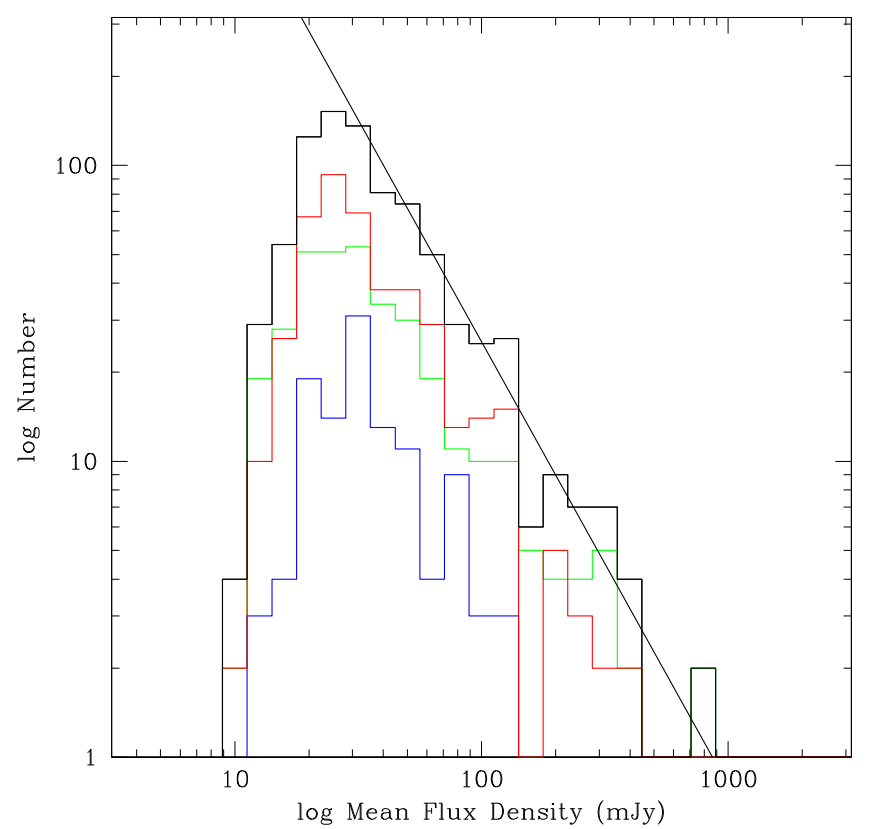

Figure 6. Log of the mean $\mathrm{H}$ I flux density $S$ (flux integral divided by the line width $w_{50}$ ) for all HIZOA-S galaxies that lie within the survey limits at which full sensitivity is reached $\left(|b|<5^{\circ}\right)$. The black line corresponds to a slope of $-3 / 2$ that would be expected for a homogeneous distribution. The colored histograms subdivide the survey area into three equal-sized intervals of $\Delta \ell=60^{\circ}$ (similar to Figure 5).

galaxies is the counterpart. In fact, we find that for $13 \%$ of our $\mathrm{H}$ idetections there is a galaxy closer than the cross-match.

\subsection{Properties of the Counterpart Sample}

With a counterpart found for $78 \%$ of the $\mathrm{H}$ I detections, we can investigate whether there are any systematics that affect the finding of counterparts. Obviously there is a dependence on extinction, although we find that the identification of counterparts is fairly independent of extinction up to $A_{\mathrm{B}} \simeq 13^{\mathrm{m}}$ owing to the available deep NIR imaging (VISTA, UKIDSS, IRSF).

Galaxies with high $\mathrm{H}_{\mathrm{I}}$ mass and broad line widths are more easily recovered than (late-type) dwarf galaxies, although this also depends on the extinction. Figure 5 shows histograms of the $\mathrm{H}_{\text {I }}$ mass of cross-identified $\mathrm{H}$ I detections dependent on the location in the ZOA (Puppis area $212^{\circ}<l<268^{\circ}$ in green; GA area $268^{\circ}<l<340^{\circ}$ in dashed red; Local Void 


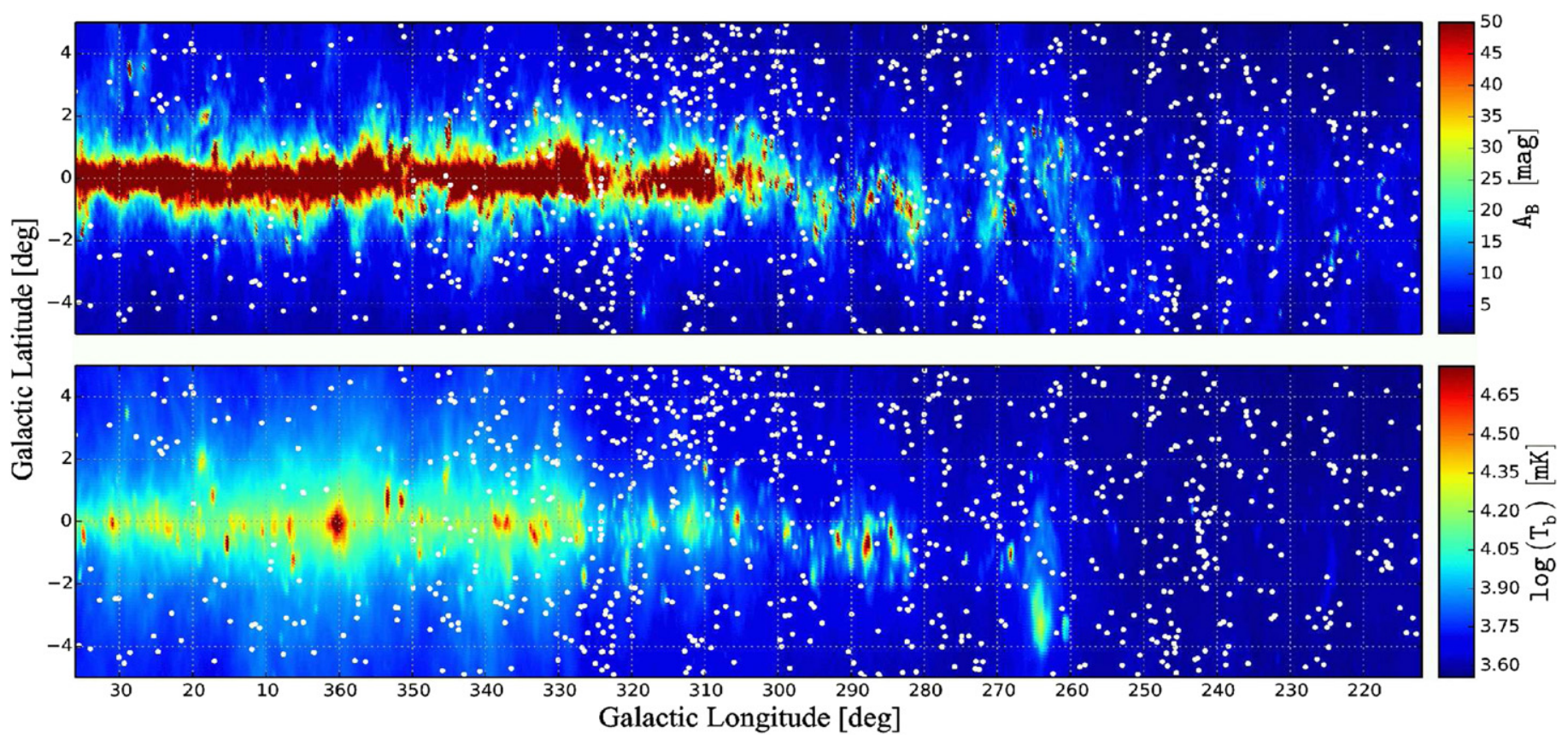

Figure 7. Distribution in Galactic coordinates of the HIZOA-S galaxies (white dots) superimposed on the recalibrated DIRBE dust extinction maps (Schlafly \& Finkbeiner 2011; top panel) and the Galactic background continuum maps (Calabretta et al. 2014; bottom panel). Note that galaxies are detected at some of the highest dust column density values, while this is not true for the highest continuum levels (see also Figure 9).

$[\mathrm{LV}]$ area $340^{\circ}<l<36^{\circ}$ in blue). In the Puppis area, far from the Galactic bulge, we find that almost all HIZOA detections have cross-matches. However, the detection rate decreases with $\mathrm{H}$ I mass for galaxies found in the $\mathrm{LV} /$ Galactic bulge area, where stellar crowding and extinction severely affect the cross-identification in the optical/NIR.

\section{COMPLETENESS, ACCURACY AND RELIABILITY}

\subsection{Completeness}

Previous analysis of the data in the otherwise identical northern extension of this survey, HIZOA-N (Donley et al. 2005), has concluded that the completeness limit lies at a mean flux density of $22 \mathrm{mJy}$. That is, galaxies with profiles whose mean flux density, $S$, is greater than $22 \mathrm{mJy}$ are generally detected with high completeness. Galaxies below this threshold can be detected, but with increasingly poor completeness. The main exceptions to this limit, as also noted by Donley et al., are in regions of high rms at the edge of the field of view and, more importantly, toward bright radio continuum regions in the Galactic plane. Bright Galactic continuum not only raises the receiver temperature and lowers sensitivity but can also give rise to non-flat spectral baselines, hampering detectability. A histogram of the mean flux density, $S$, for HIZOA-S constrained by $212^{\circ} \leqslant \ell \leqslant 36^{\circ}$ and $|b| \leqslant 5^{\circ}$ is shown in Figure 6, as well as three subsets of different Galactic longitude ranges of width $\Delta \ell=60^{\circ}$. In agreement with Donley et al., incompleteness in mean flux density is obvious below $30 \mathrm{mJy}$. Incompleteness seems to drop off more quickly in the region represented by the blue histogram, which contains the Local Void. This is probably due to a combination of the higher continuum in this region (see below) and large-scale structure.

The 883 galaxies detected in HIZOA-S are overlaid on an image of the Galactic continuum background made from the same multibeam data by Calabretta et al. (2014) in the bottom panel of Figure 7. The anticorrelation between Galactic

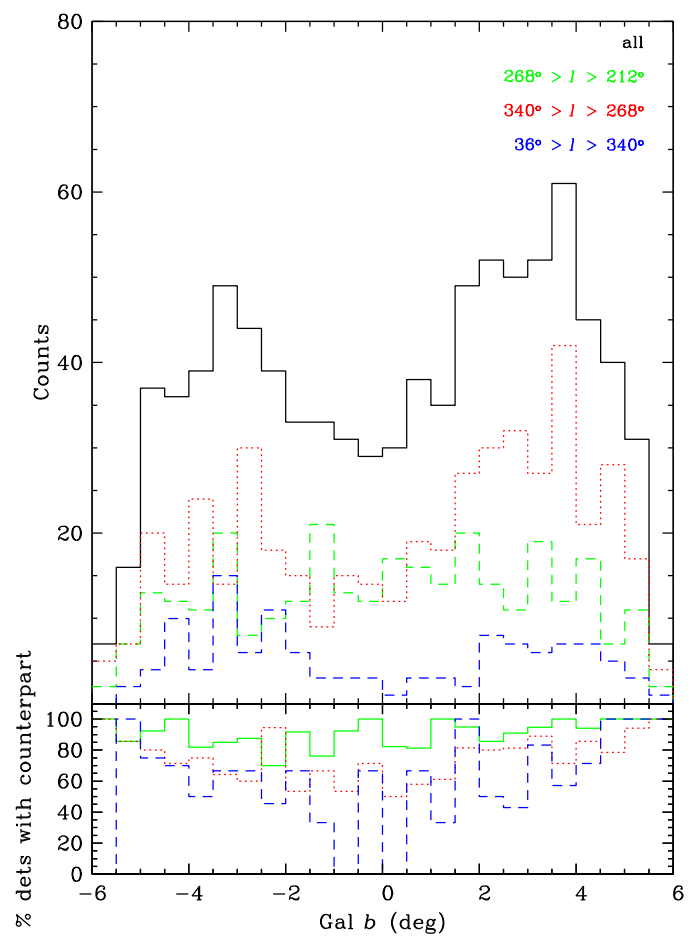

Figure 8. Number of detections plotted as a function of Galactic latitude for three different longitude ranges as indicated at the top right, and for the combined sample (solid black line). The counterpart identification rate is plotted in the lower panel.

continuum and galaxy detection is noticeable for $|b|<1^{\circ}$, although note that the Local Void (Section 7) results in reduced galaxy density at all latitudes for $\ell>350^{\circ}$. Figure 7 also shows the correlation with dust extinction (top panel). Although a broadly similar anticorrelation at $|b|<1^{\circ}$ is evident, a detailed comparison shows that galaxies can be detected at all optical 

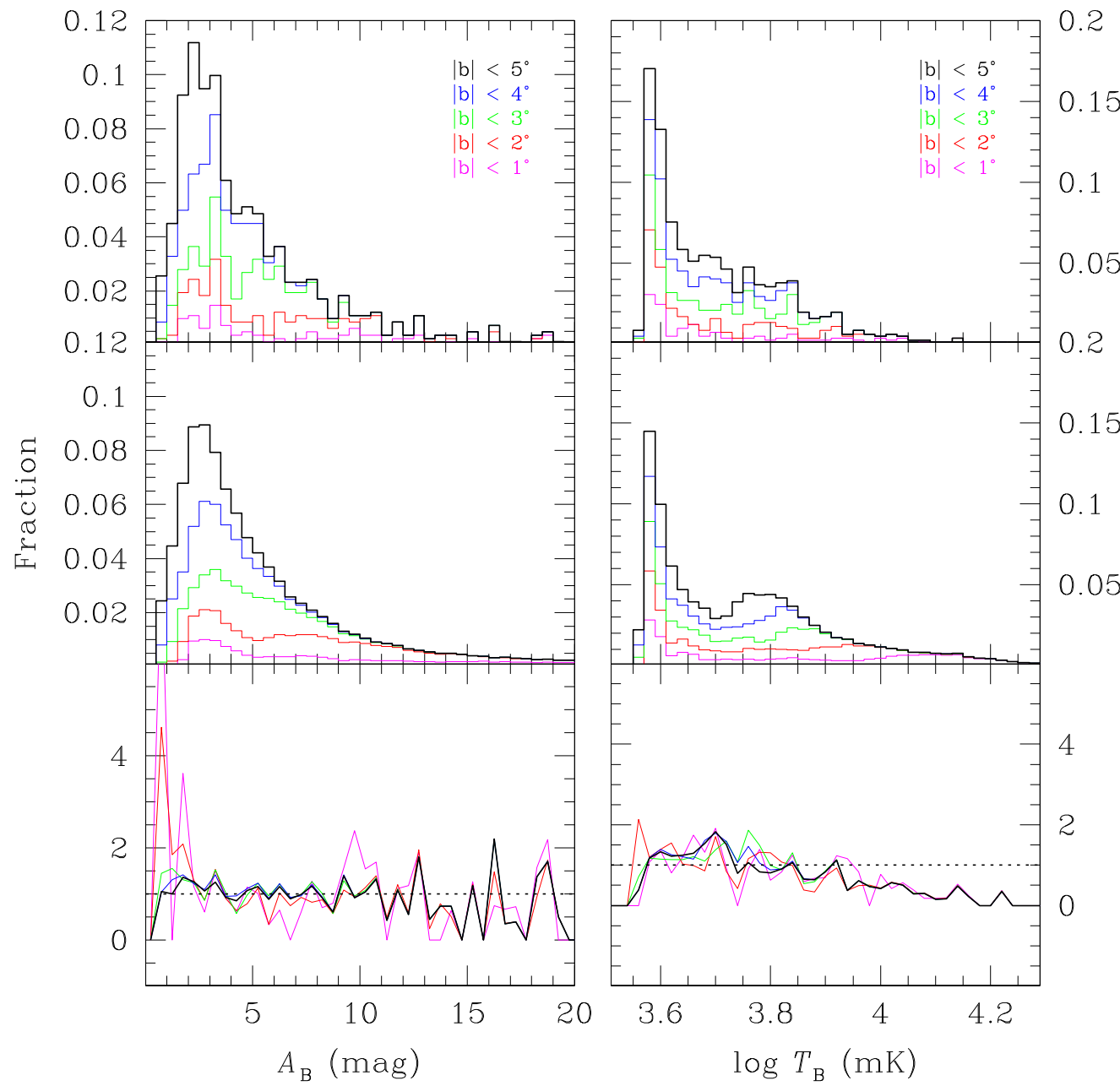

Figure 9. Detection rate as a function of foreground extinction (left panels) and continuum background (right panels). Colors represent different latitude cuts: black, blue, green, red, magenta for $|b|<5^{\circ}, 4^{\circ}, 3^{\circ}, 2^{\circ}, 1^{\circ}$, respectively. Top panels: distribution of $A_{\mathrm{B}}$ and $\log T_{\mathrm{B}}$ levels at the position of HIZOA-S galaxies as a fraction of the total number within the respective latitude limits. The continuum shows a much sharper drop-off compared to $A_{\mathrm{B}}$ (note the different scales). Middle panels: $A_{\mathrm{B}}$ and $\log T_{\mathrm{B}}$ over the surveyed area calculated from a grid of cells of $0.1 \times 0.1$. Note the smoothness of the distributions and the similarity to the distribution in the top panel, apart from a bump around $\log T_{\mathrm{B}} \sim 3.7-3.9$. This is due to the Galactic bulge and disappears completely when the region is limited to $\ell<325^{\circ}$. Bottom panels: ratio of $A_{\mathrm{B}}$ and $\log T_{\mathrm{B}}$ at the position of HIZOA-S galaxies to the overall survey values (a division of the top and middle histograms), which should be flat if there is no dependence.

Table 4

Rauzy Completeness Limits for Flux Integral, Mean Flux Density, and Scaled Fluxes

\begin{tabular}{lcccc}
\hline \hline & $\begin{array}{c}T_{c}=-3 \\
\text { Limit }\end{array}$ & $f$ & Limit & $f$ \\
Parameter & $3.1 \mathrm{Jy} \mathrm{km} \mathrm{s}^{-1}$ & 0.75 & $3.3 \mathrm{Jy} \mathrm{km} \mathrm{s}$ & 0.72 \\
\hline Flux integral, $F$ & $21 \mathrm{mJy}^{-1}$ & 0.81 & $22 \mathrm{mJy}^{-1}$ & 0.74 \\
Mean flux density, $S$ & $2.8 \mathrm{Jy} \mathrm{km} \mathrm{s}^{-1}$ & 0.92 & $2.9 \mathrm{Jy} \mathrm{km} \mathrm{s}$ & 0.90 \\
$F\left(w_{50}^{u} / 160 \mathrm{~km} \mathrm{~s}^{-1}\right)^{0.74}$ & $17 \mathrm{mJy}^{-1}$ & 0.91 & $18 \mathrm{mJy}$ & 0.90 \\
$S\left(w_{50}^{u} / 160 \mathrm{~km} \mathrm{~s}^{-1}\right)^{-0.26}$ & & & \\
\hline
\end{tabular}

Note. $T c=-3$ and $T c=-2$ correspond to the $99.3 \%$ and $97.7 \%$ confidence bounds for the completeness limits, respectively; $f$ is the fraction of galaxies above the completeness limit. Completeness limits refer to objects in the main survey region $\left(212^{\circ}<\ell<36^{\circ}, 5^{\circ}>b>-5^{\circ}\right)$ and Galactic foreground brightness $T_{\mathrm{B}}<7 \mathrm{~K}$.

extinctions (including $A_{\mathrm{B}}>50 \mathrm{mag}$ ) as long as the Galactic foreground temperature is $T_{\mathrm{B}}<20 \mathrm{~K}$. More quantitatively, the detection rate as a function of Galactic latitude is plotted in Figure 8. A deficit in detections is apparent outside the nominal latitude range $\pm 5^{\circ}$ and at $|b|<1.5$. The latter deficit is much more striking near the Galactic center. The relative surface density of galaxies found at increasing continuum temperature and optical extinction is shown in Figure 9. The anticorrelation with continuum is much tighter than for extinction, with $\sim 100 \%$ detectability for $T_{\mathrm{B}}<7 \mathrm{~K}$, decreasing to $50 \%$ at $10 \mathrm{~K}$ and to almost zero at $T_{\mathrm{B}}>16 \mathrm{~K}$, although the latter represents only $1.9 \%$ of the area surveyed. As with HIZOA-N, the reason for the low detectability in regions of bright continuum is mainly the increased baseline ripple and higher system temperature. Very little of the Galactic plane is optically thick at $1.4 \mathrm{GHz}$.

The significant numbers of galaxies detected in this survey allow a more detailed characterization of completeness than made by Donley et al. (2005). We use the technique of Rauzy (2001), which is a modified $V / V_{\max }$ test. This has previously been used for $\mathrm{H}_{\mathrm{I}}$ surveys alongside false-source injection techniques by Zwaan et al. (2004) for HIPASS and Hoppmann et al. (2015) for the Arecibo Ultra Deep Survey (AUDS) and has been shown to be a robust alternative. Its main advantage is that, compared with source counts (e.g., Figure 6), it is 

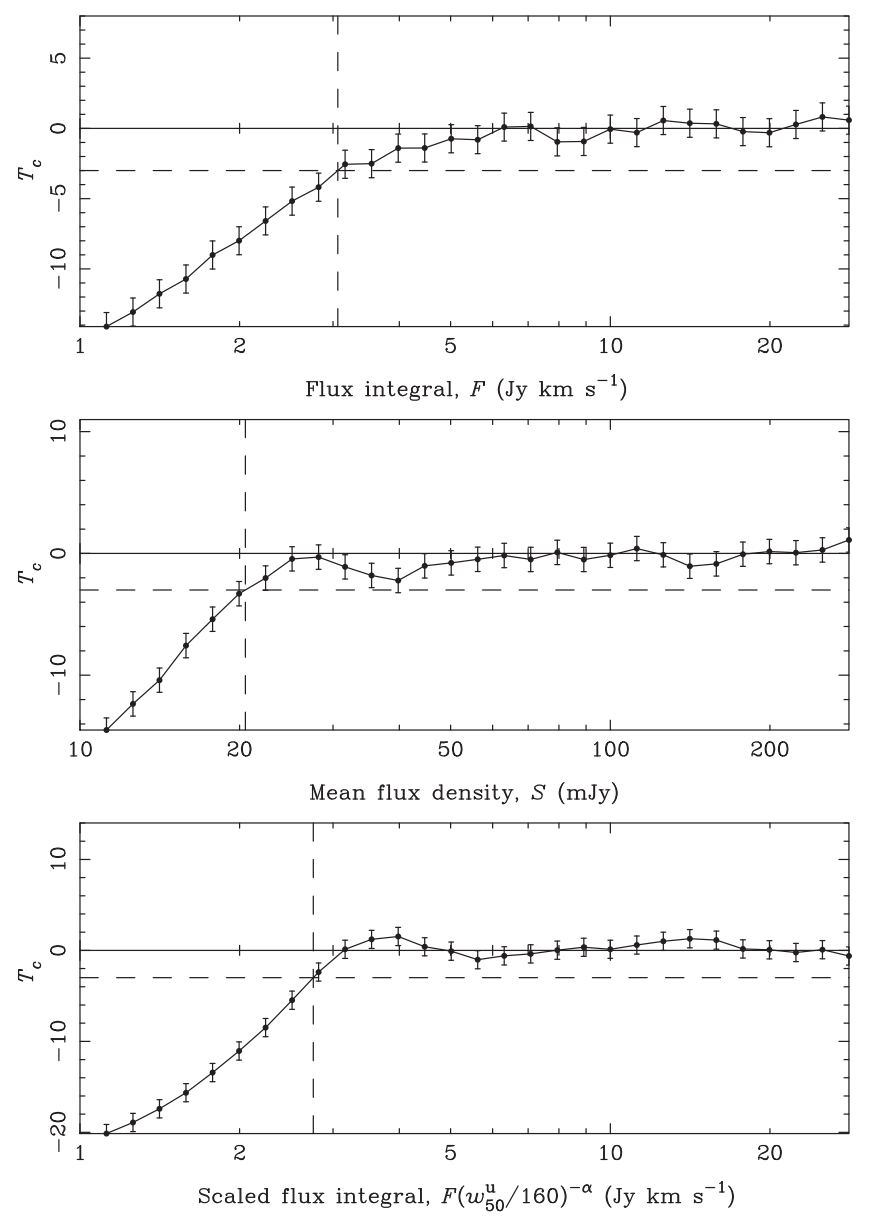

Figure 10. Rauzy completeness statistic $T_{c}$ as a function of faint cutoff limit for flux integral (top panel), mean flux density (middle panel), and scaled flux integral (bottom panel). $T_{c}$ values less than zero indicate incompleteness, with $T_{c}=-3$ corresponding to the lower $99.3 \%$ confidence bound for completeness. The best description of completeness for this survey is given by the scaled flux integral with $\alpha=0.74$.

insensitive to large-scale structure, which is considerable in the southern ZOA. Disadvantages are that (a) it does not allow the characterization of a "soft" roll-off in completeness and (b) the method assumes no substantial variation in the shape of the $\mathrm{H}$ I mass function with position, e.g., as a function of environment. Evolutionary effects (Rauzy 2001) and bright limits (Johnston et al. 2007a) can be incorporated but are unnecessary for this analysis.

Results of the Rauzy test are presented in Table 4 and Figure 10. For regions within the main survey area $\left(212^{\circ}<\ell<36^{\circ}, \quad 5^{\circ}>b>-5^{\circ}\right)$ and $T_{\mathrm{B}}<7 \mathrm{~K}$, the $T_{c}=-3$ completeness limits are $F_{\circ}=3.1 \mathrm{Jy} \mathrm{km} \mathrm{s}^{-1}$ for flux integral and $S_{\circ}=21 \mathrm{mJy}$ for mean flux density. The Rauzy test indicates that there is only $0.6 \%$ probability that the actual completeness limits are fainter than this. The mean flux density limit (defined here as the ratio of flux integral, $F$, to the velocity width prior to resolution correction, $w_{50}^{u}$ ) is satisfactorily similar to the value of $22 \mathrm{mJy}$ deduced by Donley et al. (2005). However, Figure 10 shows that the flux-integral completeness starts to reduce below $100 \%$ well above the formal completeness limit. To a lesser extent, the mean flux density completeness also rolls off. The reason for this is that, as shown in previous blind $\mathrm{H}_{\mathrm{I}}$ surveys, completeness is a function of both flux and velocity width. This is illustrated in Figure 11,
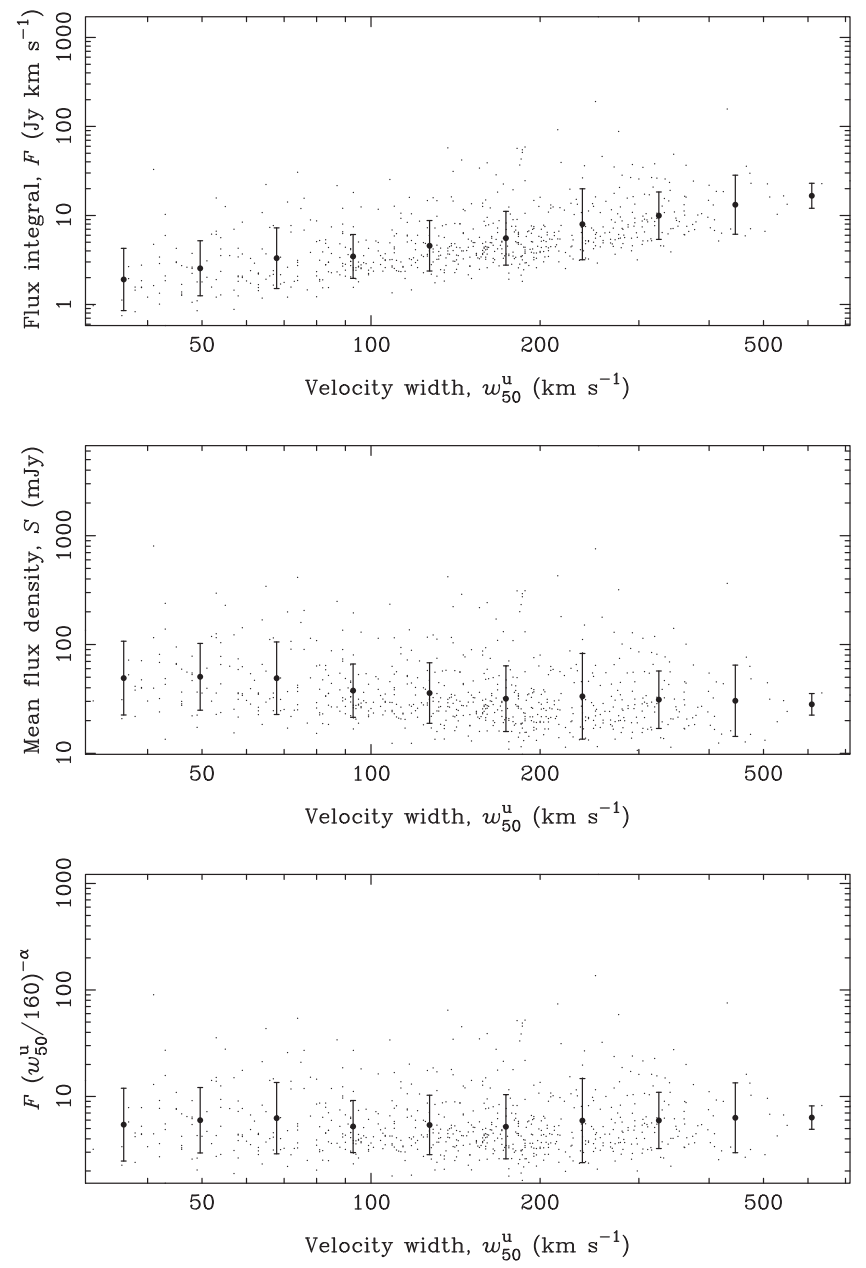

Figure 11. Measured velocity width (before resolution correction), $w_{50}^{u}$, plotted against flux integral (top panel), mean flux density (middle panel), and scaled flux integral (bottom panel) for all survey galaxies. Mean logarithmic values in 10 equally spaced intervals are shown, with error bars indicating the rms dispersion.

where galaxies of a given flux integral are clearly easier to detect at fainter limits when they have a narrow line width, $w$. Conversely, galaxies of a given mean flux density are easier to detect when they have a large line width.

The radiometer equation suggests that the flux integral selection limit should scale as $w^{\alpha}$, with $\alpha=0.5$. Conversely, the flux density selection limit should scale approximately as $w^{\alpha-1}$. However, the well-known effect of baseline ripple makes high velocity width galaxies harder to detect in practice. At Parkes, the primary ripple wavelength corresponds to $1200 \mathrm{~km} \mathrm{~s}^{-1}$. Figure 11 shows that a more appropriate index is $\alpha=0.74$, where the selection limit is characterized for flux integral by $F_{\circ}\left(w_{50}^{u} / 160 \mathrm{~km} \mathrm{~s}^{-1}\right)^{\alpha}$ and for mean flux density by $S_{\circ}\left(W_{50}^{u} / 160 \mathrm{~km} \mathrm{~s}^{-1}\right)^{\alpha-1}$. With this scaling, much tighter selection limits of $F_{\circ}=2.8 \mathrm{Jy} \mathrm{km} \mathrm{s}^{-1}$ and $S_{\circ}=17 \mathrm{mJy}$ are obtained. At a velocity width of $50 \mathrm{~km} \mathrm{~s}^{-1}$, this corresponds to flux integral and flux density limits of $1.2 \mathrm{Jy} \mathrm{km} \mathrm{s}^{-1}$ and $24 \mathrm{mJy}$, respectively. At a velocity width of $350 \mathrm{~km} \mathrm{~s}^{-1}$, this corresponds to flux integral and flux density limits of $5.0 \mathrm{Jy} \mathrm{km} \mathrm{s}^{-1}$ and $14 \mathrm{mJy}$, respectively. Thus defined, $92 \%$ of the sample is brighter than the hybrid selection limit, compared with only $75 \%$ and $81 \%$ for pure flux and flux-density-limited 
Table 5

HIPASS Detections Not in the HIZOA-S Catalog

\begin{tabular}{|c|c|c|c|c|c|}
\hline HIPASS & Gal $b$ & $\mathrm{~S} / \mathrm{N}$ & $q^{\mathrm{a}}$ & Note & Comment \\
\hline J0817-45 & $-\mathbf{5 . 5 9}$ & 9.2 & 1 & real & $\begin{array}{l}\text { Below the acceptance limit } \\
\text { (noisy area at the edge) }\end{array}$ \\
\hline J0844-38 & 2.45 & 4.6 & 2 & not real & $\ldots$ \\
\hline J0857-41 & 2.55 & 4.5 & 2 & not real & $\ldots$ \\
\hline $\mathrm{J} 1026-51$ & 5.09 & 6.2 & 1 & real & $\begin{array}{l}\text { Below the acceptance limit } \\
\text { (bad baseline at the edge) }\end{array}$ \\
\hline $\mathrm{J} 1232-68$ & $-\mathbf{5 . 3 8}$ & 5.4 & 1 & real & $\begin{array}{l}\text { Below the acceptance limit } \\
\text { (noisy area at the edge) }\end{array}$ \\
\hline J1412-65 & -4.09 & 132.4 & 1 & not real & Part of Circinus \\
\hline $\mathrm{J} 1418-63$ & -2.19 & 3.3 & 2 & not real & $\ldots$ \\
\hline J1439-54 & 5.28 & 6.0 & 1 & real & $\begin{array}{l}\text { Below the acceptance limit } \\
\text { (faintly visible at edge) }\end{array}$ \\
\hline $\mathrm{J} 1440-53$ & 5.58 & 7.8 & 1 & real & $\begin{array}{l}\text { Below the acceptance limit } \\
\text { (visible at edge) }\end{array}$ \\
\hline J1444-53 & 5.38 & 4.5 & 2 & not real & $\ldots$ \\
\hline J1516-58 & -0.50 & 3.5 & 2 & not real & $\cdots$ \\
\hline $\mathrm{J} 1600-52$ & 0.48 & 4.2 & 2 & not real & $\cdots$ \\
\hline $\mathrm{J} 1624-47$ & 1.42 & 4.0 & 1 & not real & $\ldots$ \\
\hline $\mathrm{J} 1655-49$ & -3.99 & 4.4 & 2 & not real & $\ldots$ \\
\hline J1642-37 & 5.66 & 6.3 & 1 & real & $\begin{array}{c}\text { Below the acceptance limit } \\
\text { (visible at edge) }\end{array}$ \\
\hline J1708-37 & 1.35 & 8.0 & 1 & not real & Likely RFI \\
\hline $\mathrm{J} 1740-37$ & -3.80 & 7.9 & 1 & not real & Likely RFI \\
\hline J1713-33 & 2.94 & 9.2 & 1 & not real & Likely RFI \\
\hline $\mathrm{J} 1718-27$ & 5.62 & 6.4 & 1 & real & $\begin{array}{l}\text { Below the acceptance limit } \\
\text { (faintly visible at edge) }\end{array}$ \\
\hline $\mathrm{J} 1743-21$ & 4.09 & 5.9 & 1 & real & Below the acceptance limit \\
\hline $\mathrm{J} 1823-16$ & -1.44 & 5.4 & 1 & not real & Likely RFI \\
\hline J1828-09 & 0.82 & 4.0 & 1 & not real & $\ldots$ \\
\hline J1817-04 & 5.75 & 4.6 & 1 & not real & $\ldots$ \\
\hline
\end{tabular}

Notes. Values in bold typeface represent objects outside the nominal HIZOA range in Galactic latitude, low-S/N HIPASS detections, or low HIPASS quality.

"HIPASS quality flag: $q=1=$ "real"; $q=2=$ "have concerns."

samples, respectively. Additional dependencies, such as with profile shape (Zwaan et al. 2004), are small for the hybrid limit.

\subsection{Comparison with Other H I Catalogs}

Two subsamples of the data presented here had been previously analyzed: Henning et al. (2000) published 110 bright $\mathrm{H}$ I detections based on $8 \%$ of the full integration time, and Juraszek et al. (2000) published $42 \mathrm{H}$ i detections in the GA region based on $16 \%$ of the full integration time. All of these detections were recovered. The only $\mathrm{H}_{\text {I }}$ detection with a large positional offset compared to the previous publications $\left(d>10^{\prime}\right)$ is $\mathbf{~} 1616-55$, which is an extended, multicomponent object described in detail in Staveley-Smith et al. (1998) (see also the note in the Appendix).

As is normal between adjacent cubes, there is a $1^{\circ}$ overlap around $l=36^{\circ}$ and $l=196^{\circ}$ between this catalog and its extension to the north, HIZOA-N (Donley et al. 2005). The two catalogs have three galaxies in common. A further detection, found by us at $l=36^{\circ} .06$, is technically part of HIZOA-N but was missed there.

We compared our detections with HIPASS, which is an independent survey of the southern hemisphere (Meyer et al. 2004; hereafter HIPASS-south), and its extension to the north (Wong et al. 2006; hereafter HIPASS-north) at $20 \%$ of
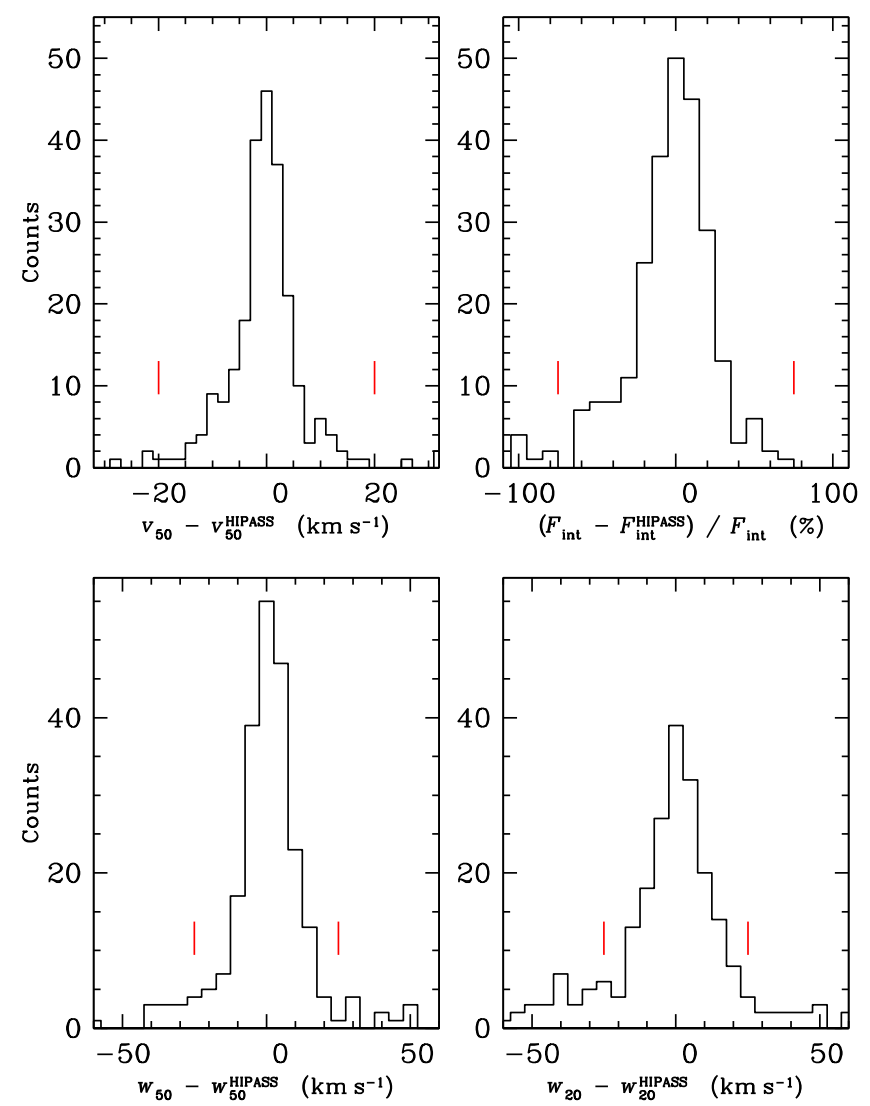

Figure 12. Histograms of differences between HIZOA-S and HIPASS parameters: $v_{50}$ (top left), flux (difference percentage; top right), $w_{50}$ (bottom left), and $w_{20}$ (bottom right). The histograms are truncated, where 18 are outside the plotted range for $v_{50}$ (out to $\left|\Delta v_{50}\right| \simeq 130 \mathrm{~km} \mathrm{~s}^{-1}$ ), 1 for flux integral (at $-177 \%$ ), 17 for $w_{50}$ (out to $\left|\Delta w_{50}\right| \simeq 380 \mathrm{~km} \mathrm{~s}^{-1}$ ), and 14 for $w_{20}$ (out to $\left|\Delta w_{20}\right| \simeq 250 \mathrm{~km} \mathrm{~s}^{-1}$ ). The vertical bars indicate the cuts used for the statistics given in Table 6 .

Table 6

Comparison of HIPASS and HIZOA-S Parameters

\begin{tabular}{lcccc}
\hline \hline Parameter & Cut & $N$ & \multicolumn{1}{c}{ Mean } & Std dev \\
\hline$v_{50}$ & $20 \mathrm{~km} \mathrm{~s}^{-1}$ & 227 & $-0.3 \pm 0.4 \mathrm{~km} \mathrm{~s}^{-1}$ & $5.6 \mathrm{~km} \mathrm{~s}^{-1}$ \\
Flux integral, $F$ & $75 \%$ & 246 & $-0.6 \pm 1.4 \%$ & $23.8 \%$ \\
$w_{50}$ & $25 \mathrm{~km} \mathrm{~s}^{-1}$ & 214 & $0.2 \pm 0.6 \mathrm{~km} \mathrm{~s}^{-1}$ & $8.8 \mathrm{~km} \mathrm{~s}^{-1}$ \\
$w_{20}$ & $25 \mathrm{~km} \mathrm{~s}^{-1}$ & 179 & $0.9 \pm 0.7 \mathrm{~km} \mathrm{~s}^{-1}$ & $9.9 \mathrm{~km} \mathrm{~s}^{-1}$
\end{tabular}

our integration time. There are 251 detections in common, only one of which is listed in HIPASS-north (J0705+02). One HIPASS detection is confused (J1000-58) and was resolved into two detections in our deeper survey. An additional two detections each are recorded in publications based on older versions of the HIPASS catalog, namely, Kilborn et al. (2002) and Ryan-Weber et al. (2002).

There are a further 23 galaxies (all in HIPASS-south) that were not detected in HIZOA-S; see Table 5. On closer inspection, we find eight detections likely to be real, of which four were detected in the visual searches but lie below our limit for inclusion (usually owing to locally high rms), while the other four are located near the edge of a cube and were thus missed owing to the higher noise (though they were also below our acceptance limit).

Of the 15 HIPASS detections considered not to be real, seven were labeled in the HIPASS catalog as "have concerns" 
(quality flag $q=2$ ), all of which have low $\mathrm{S} / \mathrm{N}(<5.0)$; they could not be confirmed in our cubes. Three further detections with $q=1$ have similarly low $\mathrm{S} / \mathrm{N}$ and could not be confirmed by us either. One HIPASS detection (J1412-65) forms part of Circinus (our J1413-65 detection). The remaining four detections seem to be caused by RFI: they are narrow peaks with high $\mathrm{S} / \mathrm{N}$ in the HIPASS spectra, but nothing is evident in the HIZOA cubes.

In Figure 12 we compare the parameters of the 254 detections in common (excluding J1000-58). For HIPASS we use their width-maximized $v_{50}, w_{50}$, and $w_{20}$. Note that not all objects have an entry for these parameters, while our catalog also misses some of the $w_{20}$ parameters. Outliers are generally due to two reasons: (1) confused or lopsided profiles (all extreme cases fall into this category) and (2) noisy profiles or profiles affected by a poor baseline. Table 6 gives the mean and standard deviations of the "core" of the histograms shown in Figure 12 (indicated with red vertical bars). In summary, we see no statistically significant systematic effects.

We have also compared our detections with the deeper, pointed Parkes survey of southern ZOA galaxies by KraanKorteweg et al. (2002) and Schröder et al. (2009), which has a typical rms of 2-6 mJy (hereafter referred to as the PKS sample). We have 39 detections in common. In six cases the ID given in the PKS sample seems to be misjudged (J1222-57, J1337-58B [confused profile], J1405-65, J1550-58, J1553 -61, J1612-56; see Appendix for details), which is mostly due to off-beam detections in the high galaxy density area in the GA region. The 23 detections in the PKS sample not recovered by us are usually below the HIZOA sensitivity limit or lie near the edge of our survey area and are thus lost in the noise $(N=20)$. Three PKS detections were detected in our visual searches but were just under the acceptance limit.

A comparison of parameters gives similar values of mean and scatter as for HIPASS though with larger uncertainties due to the smaller number of samples. Outliers are due to confused profiles $(N=4)$ or low $\mathrm{S} / \mathrm{N}$ and/or baseline variations $(N=2)$.

We have also used HyperLEDA to extract all galaxies with $\mathrm{H}$ I velocities within our survey region. Next to the HIPASS and PKS detections discussed above, there are $39 \mathrm{H}$ i detections not detected by us. Of these, 31 are too faint or too close to the survey limits to be detectable by us. Four of these detections were found in the visual searches of the HIZOA cubes but were under the acceptance limit. Two detections seem to be off-beam detections of one of our detections: J0806-27 was attributed to PGC 022808 by Matthews \& Gallagher (1996), and J0749 -26B was correctly attributed to CGMW 2-1330 by Matthews et al. (1995), but with the wrong coordinates (that is, it was cross-matched with PGC 100722 in HyperLEDA). Two bright detections (with $F>50 \mathrm{Jy} \mathrm{km} \mathrm{s}^{-1}$ ) could not be confirmed by us: ESO 494-013 (reported by Kraan-Korteweg \& Huchtmeier [1992], and which we detected as J0802-22 at a different velocity) and PGC 2815809 (Huchtmeier et al. 2001); they are both detections with the Effelsberg Radio Telescope and possibly RFI.

\subsection{Reliability}

Within the boundaries of the full-sensitivity survey and the source detection adjudication process (Section 3), the sample is expected to be nearly $100 \%$ reliable (see Donley et al. 2005). The high reliability was ensured by a reasonably high cutoff in $\mathrm{S} / \mathrm{N}$. No marginal detections were included in the catalog, though follow-up observations are planned to confirm some of these.

We have conducted two checks on the reliability:

i. Of 56 detections in the overlap regions between adjacent cubes, only 2 were not detected in both cubes: J1847+04 belongs nominally to the northern extension, and J0818 -33 is faint and was not recognized in one cube owing to large baseline variations near the edge of the cube.

ii. We have cross-checked our detections with the nondetections in the deeper (but pointed) Parkes survey of optically selected galaxies in the southern ZOA by KraanKorteweg et al. (2002) and Schröder et al. (2009) and found no false positives (with the caveat that a pointed survey has a different selection function than a blind survey).

\section{LARGE-SCALE STRUCTURE}

In this section we investigate the large-scale distribution of the galaxies detected in this survey and discuss newly identified structures in the context of known large-scale structures in the immediate vicinity. For the latter we use publicly available data archives like HyperLEDA (Paturel et al. 2003) and, for some discussion, the 2MASS Redshift Survey (2MRS; Huchra et al. 2012). We combine our survey with the northern extension (HIZOA-N; Donley et al. 2005). That survey was observed with the same telescope using an identical strategy and analyzed in exactly the same manner by the same team. The structures identified in HIZOA-N (two $16^{\circ} \times 10^{\circ}$ fields) can be better appreciated by combining with the current HIZOA-S catalog. The combined HIZOA survey data cover the Galactic longitude range $52^{\circ}>\ell>196^{\circ}\left(\Delta \ell=216^{\circ}\right)$ for the latitude range of $|b|<5^{\circ}$ (see Figure 13).

Duplicates from overlap regions in the two surveys were eliminated. As discussed in Section 6.2, three galaxies were published in Donley et al. (2005) but nominally belong in the current HIZOA-S catalog, while there was one galaxy identified in the HIZOA-S cubes that technically belongs in HIZOA-N but was not listed there. This means that the total number of galaxies in the merged list sums to $N=957$ rather than $N=960(883+77)$.

For the large-scale-structure discussion, all HIZOA galaxies will be used. Note, however, that 62 galaxies lie just beyond the nominal latitude limit $\left(|b|>5^{\circ}\right)$ and a further one outside the longitude limit. The total number in the full-sensitivity survey area, away from the edges, is therefore $N=894$ galaxies, which translates to an average density of 0.41 galaxies per square degree for the nominal survey area of $2160 \mathrm{deg}^{2}$.

\section{1. $2 D$ and Redshift Distributions of the HIZOA Galaxies}

Figure 13 displays the distribution around the Galactic plane of the 957 galaxies detected in the HIZOA survey. The survey areas are marked by the dashed line. The 63 galaxies that lie just beyond the nominal survey borders are easily identifiable. The resulting galaxy distribution shows remarkable substructures. These stand out even when ignoring the color-coding of the dots, which symbolizes different redshift ranges (cyan: $500-3500 \mathrm{~km} \mathrm{~s}^{-1}$; blue: $3500-6500 \mathrm{~km} \mathrm{~s}^{-1}$; red: $6500-9500 \mathrm{~km} \mathrm{~s}^{-1}$; black: $9500-12500 \mathrm{~km} \mathrm{~s}^{-1}$ ). The right part of the plot shows a fairly smooth distribution of galaxies with an inkling of a filamentary feature crossing the 


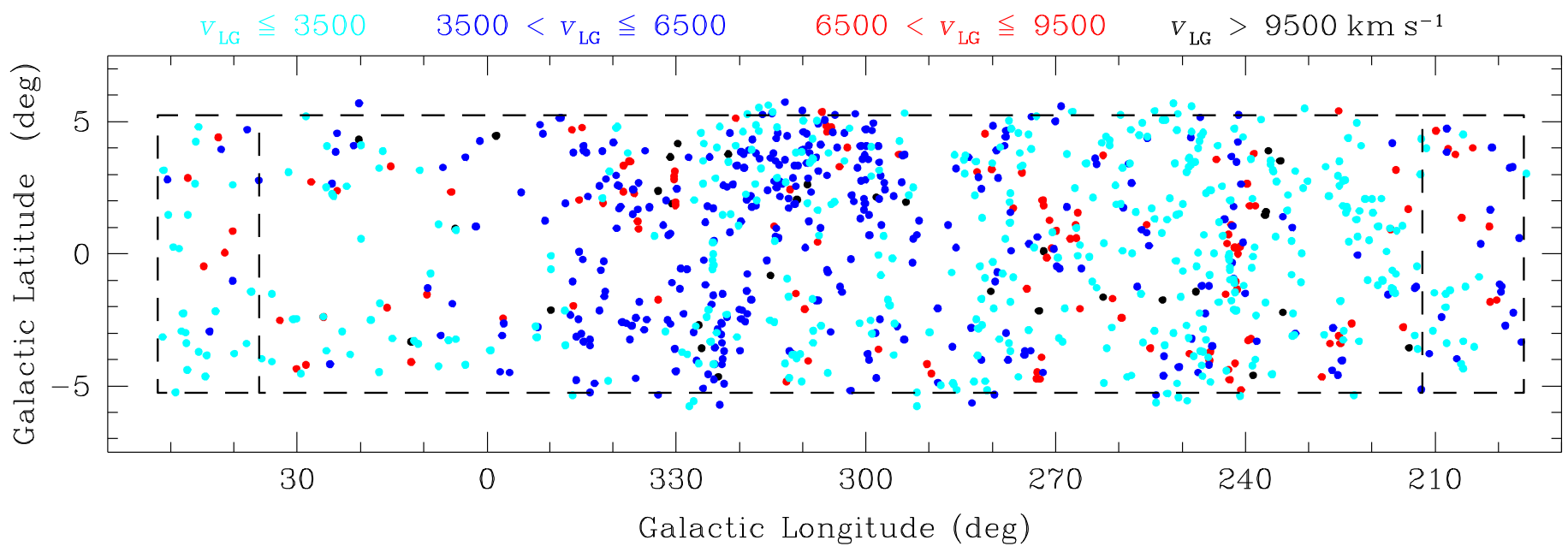

Figure 13. Distribution in Galactic coordinates of the $957 \mathrm{H}$ I-detected galaxies in the merged HIZOA catalog: HIZOA-S $\left(36^{\circ}>\ell>212^{\circ},|b|<5^{\circ}\right)$ and $\mathrm{HIZOA}-\mathrm{N}$ $\left(196^{\circ}<\ell<212^{\circ}\right.$ and $\left.36^{\circ}<\ell<52^{\circ}\right)$. The survey areas are indicated by the dashed lines. The dots are color-coded as a function of velocity. Note the predominance of galaxies in the general GA region near $\ell \sim 312^{\circ}$ and the overall variation of large-scale structure as a function of longitude (see also Figure 14).

Galactic plane vertically in Puppis at about $\ell \sim 245^{\circ}$. This part has an average detection rate $\left(0.44 \mathrm{deg}^{-2}\right)$ similar to the mean of the survey. The middle part shows a clear density enhancement $\left(0.6 \mathrm{deg}^{-2}\right)$ that is associated with the Great Attractor (GA), whereas the left part, centered around the Galactic bulge, has few galaxies, particularly at the lowest latitudes. The density of the detections is about half of that in the Puppis region and a third of that in the GA region. This is mostly due to the dominance of the LV (Tully \& Fisher 1987; Kraan-Korteweg 2005) rather than a bias due to its location behind the Galactic bulge. As discussed in Section 6, there is no dependence of the detection rate on foreground extinction levels, and only a minor reduction where the brightness temperature is elevated $\left(T_{\mathrm{B}} \gtrsim 7 \mathrm{~K}\right)$. Only a small part of the survey area, generally limited to $|b| \lesssim 1.0$, has a higher detection threshold (see also Figures 7 and 9).

The variation of the detection rate is not the only systematic apparent in Figure 13. The mean redshifts also show a striking difference as a function of longitude. The general Puppis area is dominated by nearby structures (cyan). The area centered on the GA is dominated by higher velocities (dark blue), particularly for longitudes of $\left(290^{\circ}-340^{\circ}\right)$. The few galaxies uncovered at $340^{\circ} \lesssim \ell \lesssim 52^{\circ}$ are mainly nearby but with some at higher redshift (red and black), suggestive of being at the far side of the LV.

This can be better appreciated in Figure 14, which shows the overall velocity histogram (top panel), together with three velocity histograms subdivided in longitude ranges of width $\Delta \ell=72^{\circ}$ each. The histogram of the whole survey displays a fairly steep rise up to velocities of about $3000 \mathrm{~km} \mathrm{~s}^{-1}$, followed by a gradual drop-off toward the highest velocities, which is consistent with expectations from the loss of sensitivity at higher redshifts. A distinct peak around $5000 \mathrm{~km} \mathrm{~s}^{-1}$ superimposed on the gradual drop-off is due to the GA overdensity (see third panel). Note that galaxies are found all the way out to the velocity limit of the survey and thus probe the local volume considerably deeper than HIPASS (Meyer et al. 2004).

In the second panel, the LV clearly dominates. There are very few galaxies up to about $4500 \mathrm{~km} \mathrm{~s}^{-1}$, and only one below $1500 \mathrm{~km} \mathrm{~s}^{-1}$. A broad peak ranging from about 4500 to $6500 \mathrm{~km} \mathrm{~s}^{-1}$ seems to demarcate the right-hand side of the boundary of the $\operatorname{LV}\left(\ell \sim 345^{\circ}\right.$; see Figure 15$)$, as well as the far side of the LV (see also Figures 15 and 16). A more detailed discussion on the extent of the LV can be found in KraanKorteweg et al. (2008), including preliminary data from an extension of the deep Parkes multibeam $\mathrm{H}_{\mathrm{I}}$ survey to higher latitudes around the Galactic bulge. At higher velocities the numbers drop down to extremely low levels again, indicative of a further underdense region behind the LV, which probably is quite extended on the sky, because it is visible also in the third panel.

The third panel with its strong peak at about $5000 \mathrm{~km} \mathrm{~s}^{-1}$ is clearly dominated by the Norma Supercluster (Woudt \& Kraan-Korteweg 2000; Woudt et al. 2004; Radburn-Smith et al. 2006). At lower velocities the numbers are also elevated. This can be explained by the nearer filament that crosses the plane at about $3000 \mathrm{~km} \mathrm{~s}^{-1}$ and links up with the Centaurus clusters at higher latitudes. The number counts at higher redshifts are particularly low, and the Norma overdensity seems well separated from other structures. This is not unexpected for a cosmic-web-like universe where underdense regions are surrounded by wall-like structures.

The bottom panel is dominated by low-velocity galaxies $\left(v_{\mathrm{LG}} \lesssim 3000 \mathrm{~km} \mathrm{~s}^{-1}\right)$. They form part of the quite distinct and much larger filament that crosses the Galactic plane in Puppis. It will be discussed in more detail in Figure 16, which merges the new detections with known features beyond the ZOA. The slight overdensity around $7500 \mathrm{~km} \mathrm{~s}^{-1}$ is real and seems suggestive of a more distant filament, one that has already been highlighted as such in early ZOA H I work by Chamaraux \& Masnou (2004).

\subsection{New Large-scale Structures and Their Connectivity}

In the following we give a qualitative description of new structures detected in HIZOA and how they link and contribute to known structures based on the finalized $\mathrm{H}$ I data set. A few results have been previously presented based on preliminary catalogs (e.g., Henning et al. 2005; Kraan-Korteweg 2005; Kraan-Korteweg et al. 2005). A more quantitative analysis will be given in a subsequent paper; also included will be (a) a further extension of the Parkes ZOA surveys to higher latitudes around the Galactic bulge $\left( \pm 10^{\circ}\right.$ for the longitude range $36^{\circ}>\ell>332^{\circ}$, reaching up to higher positive latitudes 


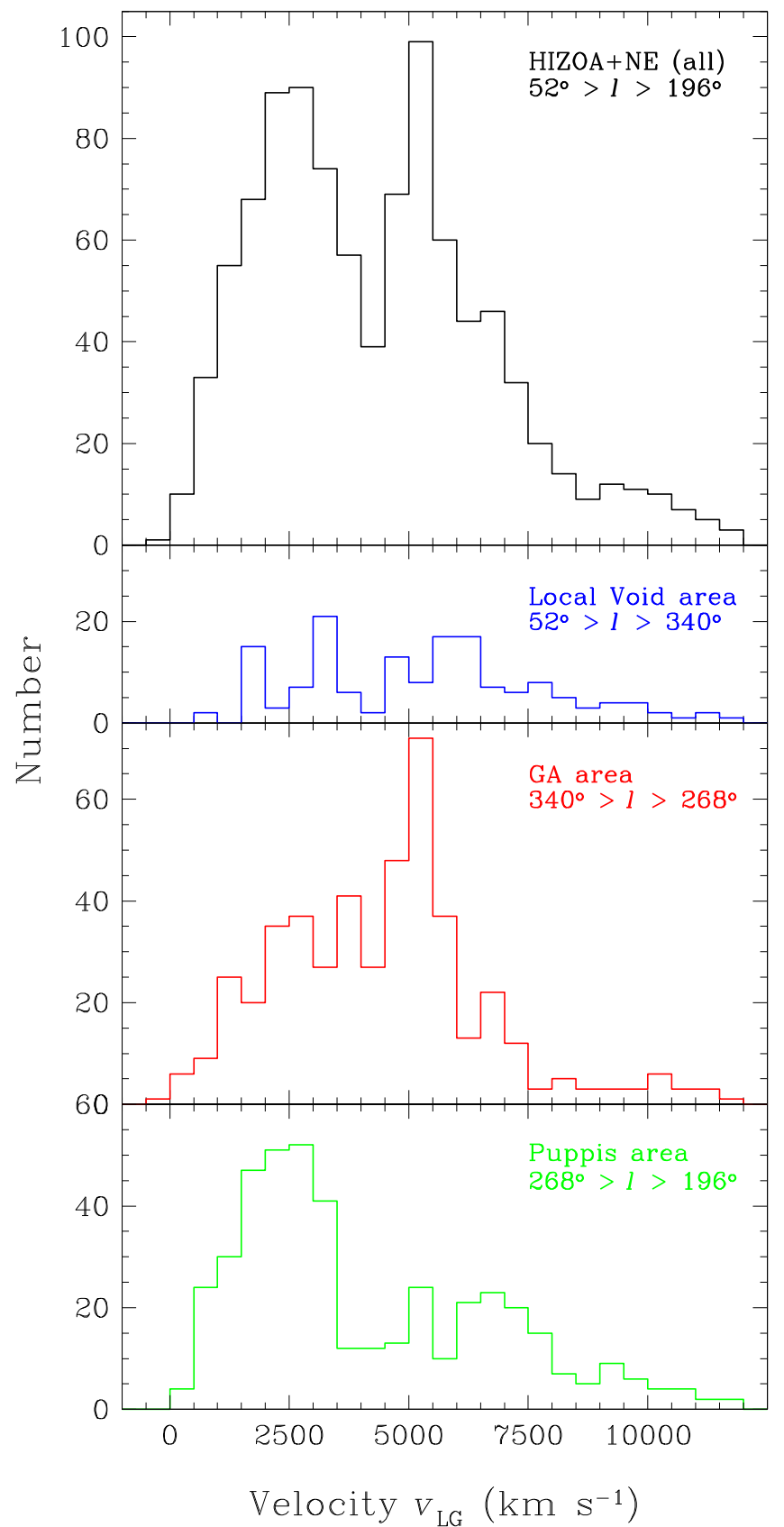

Figure 14. Velocity histogram $\left(v_{\mathrm{LG}}\right)$ of the galaxies detected in the combined HIZOA survey $\left(52^{\circ}>\ell>196^{\circ}\right)$. The top panel shows all galaxies $(N=957)$; the subsequent panels are subdivided into three regions of equal width $\left(\Delta \ell=72^{\circ}\right)$. Each one describes and encompasses vastly different structures, namely, the Local Void (LV; $N=154)$, the Great Attractor (GA; $N=462)$, and the Puppis region $(N=341)$.

$\left[+15^{\circ}\right]$ for $20^{\circ}>\ell>348^{\circ}$ ), where both the deep optical and NIR 2MASS surveys fail at identifying galaxies owing to stellar crowding, and (b) NIR counterparts of all HIZOA galaxies based on systematic deep NIR $\left(J H K_{S}\right)$ follow-up imaging observations (Williams et al. 2014, Said et al. 2015, in preparation).

We investigate the large-scale structures by examining the redshift cone defined by the HIZOA galaxies (Figure 15), and we will refer to on-sky distributions for various redshift intervals at the same time (Figure 16).
Figure 15 presents a redshift wedge with the HIZOA galaxies. The width of the wedge corresponds to the HIZOA survey width and includes all galaxies detected up to $|b| \leqslant 5^{\circ} .25$, the most opaque part of the Milky Way. It traces the structures along its full longitude range out to the velocity limit of $v_{\mathrm{LG}}=12,000 \mathrm{~km} \mathrm{~s}^{-1}$. Hardly any of these galaxies were known before the HIZOA survey, apart from a handful of galaxies in the Puppis area where the dust column density is particularly low. The high efficiency of tracing large-scale structures with systematic $\mathrm{H}$ I surveys without hindrance by the foreground "pollution" of our Milky Way-which biases most other multiwavelength surveys (e.g., Kraan-Korteweg \& Lahav 2000)—is clearly demonstrated.

Figure 16 shows on-sky distributions in Galactic coordinates centered on the southern Galactic plane. The Hi-detected galaxies are plotted together with galaxies surrounding the ZOA using redshifts extracted from HyperLEDA ${ }^{23}$ (Paturel et al. 2003) for the Galactic latitude range of $|b|<45^{\circ}$ to allow the interpretation of the newly revealed features in context with known large-scale structures. It should be emphasized that the merged samples are very differently selected. The HyperLEDA sample contains redshifts purely based on their availability in the literature and thus does not provide homogenous coverage. It also contains both radio and optically determined redshifts. Restricting the data to $\mathrm{H}_{\mathrm{I}}$ redshifts only results in an extremely shallow coverage-except for the Arecibo decl. rangeshowing again the urgent need for the forthcoming SKA Pathfinder H i surveys. Another comparison sample could have been the 2MRS (Huchra et al. 2012), which is uniform and allsky down to latitudes of $|b|>5^{\circ}$. However, the $\mathrm{H}_{\mathrm{I}}$ - and NIRselected surveys favor vastly different populations, gas-rich bluish versus early-type red galaxies, which leads to vastly different selection functions. The HIZOA galaxies are densest at low velocities because HIZOA is so sensitive to gas-rich nearby dwarfs, which $2 \mathrm{MRS}$ does not easily detect, whereas the 2MRS numbers steeply rise with redshift and the balance shifts rapidly. We hence prefer to use the HyperLEDA data sample to display the connectivity of structure across the Milky Way, because overall it provides the deepest data set and hence the optimal source to delineate structure: voids remain empty if real, and filaments will only appear more pronounced when sampled more deeply.

The panels in Figure 16 show three contiguous shells in velocity space, each of width of $3000 \mathrm{~km} \mathrm{~s}^{-1}$. Colors indicate finer redshift intervals within each panel. This figure also demonstrates quite clearly how well the inner $\pm 5^{\circ}$ of the ZOA has been filled by the HIZOA survey $(N=408, N=368$, and $N=134$ redshifts from top to bottom, respectively). The last shell out to the velocity limit of the survey is not plotted here. Owing to the low sensitivity in that redshift interval, the detection rate is too low $(N=36$; see also Figure 14) to add much insight into larger structures; their distribution can be inspected in the redshift slice (Figure 15).

As before, we will focus on the three areas separately that are dominated by quite different large-scale structures, i.e., Puppis, $\mathrm{GA}$, and LV.

\subsubsection{The Puppis Region}

The right-hand side of Figure 15 is quite crowded, particularly at the low velocity range in the Puppis region

\footnotetext{
${ }^{23}$ http://leda.univ-lyon1.fr/
} 


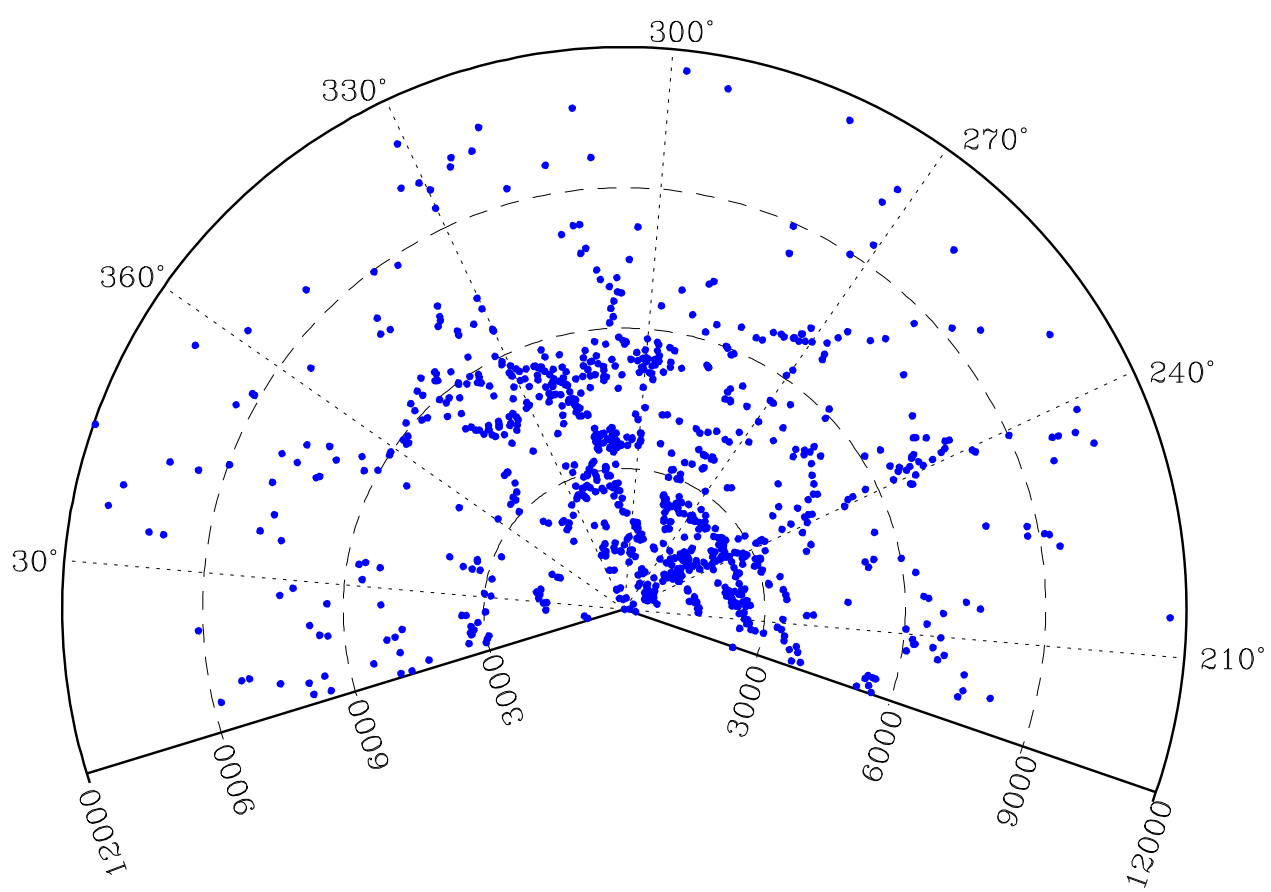

Figure 15. Redshift wedge plot for $v_{\mathrm{LG}}<12,000 \mathrm{~km} \mathrm{~s}^{-1}$ for the latitude range $|b|<5^{\circ} .25$ for the $\mathrm{H}$ I-detected galaxies in the HIZOA survey. Note the wall-like structure of the GA, the Norma Supercluster, at about $\sim 4800 \mathrm{~km} \mathrm{~s}^{-1}$ that stretches in the range $345^{\circ} \gtrsim \ell \gtrsim 290^{\circ}$.

$\left(\ell \sim 245^{\circ}\right)$. For a better visualization of these quite nearby structures we show a zoom-in version of the redshift slice in Figure 17. A nearby group and a slightly more distant small cluster at 700 and $1400 \mathrm{~km} \mathrm{~s}^{-1}$, respectively, dubbed the Puppis 1 group and the Puppis 2 cluster, stand out. Both were found already in 1992 by Kraan-Korteweg \& Huchtmeier (1992) through $\mathrm{H}_{\mathrm{I}}$ follow-up observations of optically visible galaxies in this area of low foreground extinction. Owing to their proximity, both of these galaxy concentrations were also identified and described in the shallow survey (Henning et al. 2000) and in the $\mathrm{H}_{\text {I }}$ Bright Galaxy Catalog (Koribalski et al. 2004). At slightly higher redshifts we note the Puppis filament. There is a third concentration (not marked by a circle) at slightly higher longitudes and redshifts $\left(\ell \sim 255^{\circ}, v \sim 1700 \mathrm{~km} \mathrm{~s}^{-1}\right)$, but it does not stand out as a group in three dimensions. Between 2000 and $3000 \mathrm{~km} \mathrm{~s}^{-1}$ we see a surprisingly narrow filament (henceforth referred to as the ZOA filament) that can be traced along the full longitude range of the Puppis wedge. It is well separated from other structures apart from where this filament crosses the Puppis filament $\left(\ell \sim 245^{\circ}\right)$ and the Hydra Wall $\left(\ell \sim 278^{\circ}\right)$. The Monocerus group ( $\ell \sim 220^{\circ}$; marked as a green oval) was identified in Donley et al. (2005). They suggested that the group might form part of the very narrow ZOA filament (see Figure 16). A closer look at the Puppis cone presented here (Figure 17) does not, however, entirely support this because they are distinct in redshift space.

The Puppis filament itself is a highly interesting feature; it is part of a very extended structure and can be traced over most of the southern sky (see top panel of Figure 16). From far below the Galactic plane $(\ell, b, v) \sim\left(210^{\circ},-25^{\circ}, 2800 \mathrm{~km} \mathrm{~s}^{-1}\right)$, it extends toward the ZOA around Puppis $\left(\ell \sim 245^{\circ}\right)$, crossing at slightly lower redshifts. From there it continues and connects with Antlia $\left(273^{\circ}, 19^{\circ}, 2800 \mathrm{~km} \mathrm{~s}^{-1}\right)$. It continues across Antlia toward the Centaurus Cluster $\left(302^{\circ}, 21^{\circ}, 3400 \mathrm{~km} \mathrm{~s}^{-1}\right)$ and then folds back, crossing the Milky Way once more-this second crossing lies just in front of the Great Wall or Norma Wall crossing discussed in the next section. The filament traces a near-perfect sine-wave-like structure in this projection, while in an equatorial projection it appears more circular. Its threedimensional shape seems to be a long, straight filament. Interesting, though, is the length over which the filament is contiguous across the sky $\left(\sim 180^{\circ}\right)$, certainly in comparison to its narrow width. At a mean redshift of $\sim 3000 \mathrm{~km} \mathrm{~s}^{-1}$ this translates to about $\sim 100 h^{-1} \mathrm{Mpc}$. Although interspersed with numerous galaxy groups, the filament is only $\sim 5 h^{-1} \mathrm{Mpc}$ wide, very different compared to the very broad, foam-like Norma Wall structure.

Various other filaments seem to intersect with the Puppis filament. The most prominent is a filamentary connection emanating from the Hydra Cluster $\left(270^{\circ}, 26^{\circ}, 3500 \mathrm{~km} \mathrm{~s}^{-1}\right)$ toward Antlia (where it crosses the Puppis filament), from where it moves downward in Figure 16, crossing the Galactic plane at about $\ell \sim 280^{\circ}$. This continuation to latitudes of about $-10^{\circ}$ was surmised early on by Kraan-Korteweg (1989) and Kraan-Korteweg et al. (1995) and now is substantiated with the present data set. The signature of the crossing is prominent, too, in Figure 17. It is conceivable that the Monocerus group $\left(\ell \sim 220^{\circ}\right)$ lines up with Antlia as well, but the sparsity of data in the Galactic latitude range between $+5^{\circ}$ and $+10^{\circ}$ precludes such a confirmation.

At higher redshifts (Figure 15) we see a further indication of a possible cluster (Puppis 3) at about $(\ell, v) \sim$ $\left(242^{\circ}, 7000 \mathrm{~km} \mathrm{~s}^{-1}\right)$. It is very prominent in the middle and bottom panels of the sky distributions and appears to be embedded in a filamentary structure within the survey area. This overdensity is also picked up by Chamaraux \& Masnou (2004) in their analysis of the Puppis Wall. They claim the existence of a large void between the Puppis Wall and this galaxy agglomeration. However, this is not evident from the 

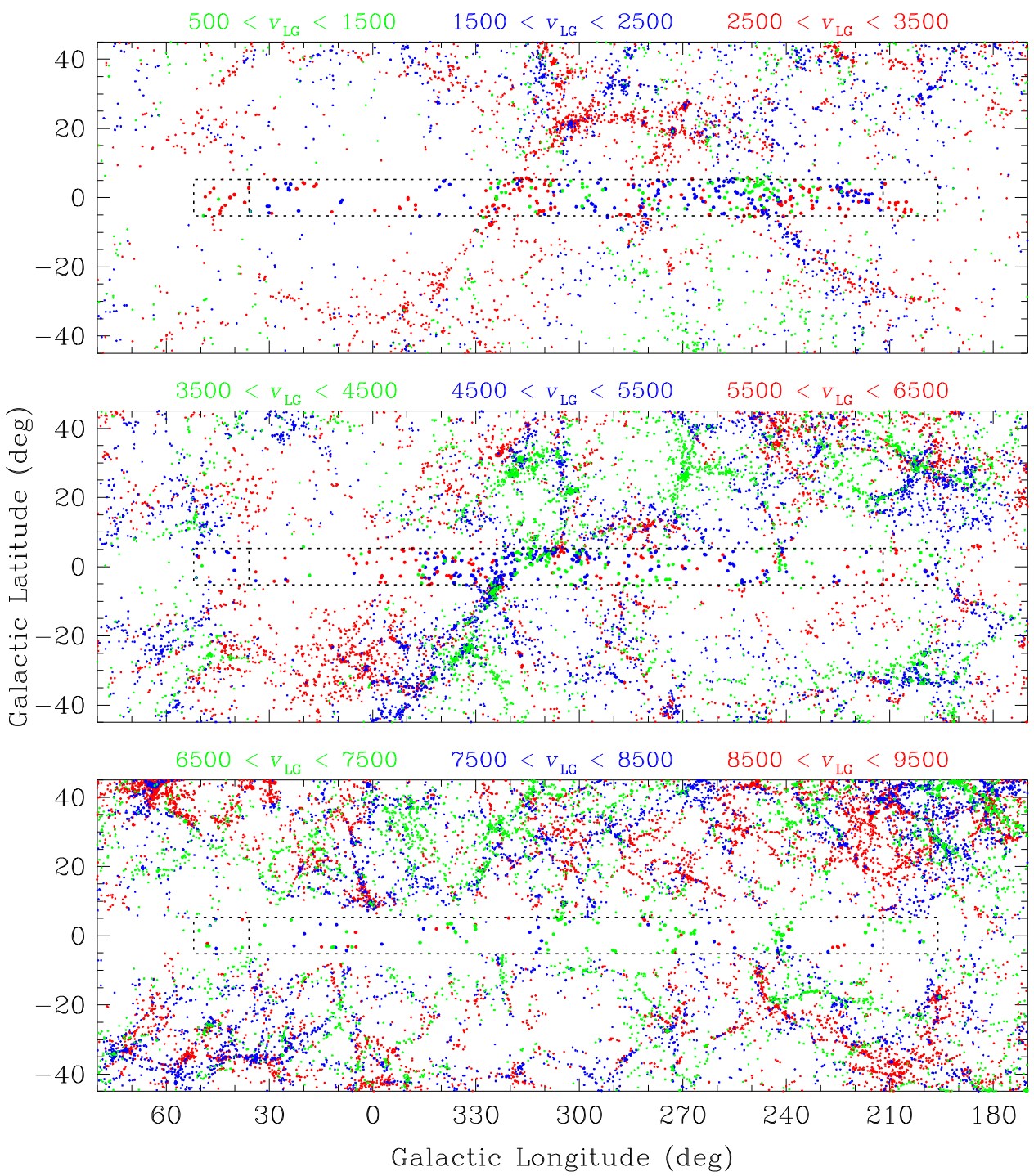

Figure 16. Three sky projections in Galactic coordinates in redshift intervals of $\Delta v=3000 \mathrm{~km} \mathrm{~s}^{-1}$ within $500<v_{\mathrm{LG}}<9500 \mathrm{~km} \mathrm{~s}^{-1}$. The survey areas are outlined. The most distant slice $\left(9500<v_{\mathrm{LG}}<12000 \mathrm{~km} \mathrm{~s}^{-1}\right)$ is not presented owing to the scarcity of detections at those redshifts $(N=36$; but see Figure 15). The HIZOA detections (larger dots) are combined with redshifts available in LEDA up to latitudes of $|b|<45^{\circ}$ to investigate the connectivity of newly revealed large-scale structures with known structures. Within the individual panels, the color-coding demarcates narrower velocity intervals $\left(\Delta v=1000 \mathrm{~km} \mathrm{~s}^{-1}\right)$.

figures presented here, which show numerous galaxies in between these redshift ranges (see Figure 15). Furthermore, the clumping or filament at about $7000 \mathrm{~km} \mathrm{~s}^{-1}$ seems to be located in the middle of a large underdense region of an extent of nearly $40^{\circ} \times 40^{\circ}$.

\subsubsection{The Great Attractor Region}

Overall, the large-scale structures revealed by the $\mathrm{HI}$ detections in Figure 15 are clearly dominated by the GA, also referred to as the Norma Wall or the Norma Supercluster (Kraan-Korteweg et al. 1994; Woudt et al. 1999, 2004; Radburn-Smith et al. 2006; Jarrett et al. 2007; Kraan-Korteweg et al. 2011, p. 98). The Norma Wall is centered on the Norma Cluster, ACO 3627 (Abell et al. 1989), which has been identified as the most massive cluster in the GA region (KraanKorteweg et al. 1996; Woudt et al. 2008). However, the Norma Cluster itself $(\ell, b, v)=\left(325^{\circ} .3,-7.2,4871 \mathrm{~km} \mathrm{~s}^{-1}\right)$ lies just outside the boundaries of our HIZOA survey. The Norma Wall crosses the ZOA diagonally-from the Norma Cluster to the
CIZA and Cen-Crux Clusters on the opposite side of the ZOA (discussed below). The HIZOA survey traces some of its previously partly, or fully, obscured components in more detail and has uncovered further galaxy concentrations that form part of the Norma Supercluster, adding to the general overdensity in this part of the sky. A zoom-in redshift cone of the GA region is presented in Figure 18.

In this plot, the Norma Wall seems to stretch from $360^{\circ}$ to $290^{\circ}$, lying always just below the $6000 \mathrm{~km} \mathrm{~s}^{-1}$ circle. Indeed, Woudt et al. $(2004,2008)$ have shown that both the Norma Cluster and the wall in which it is embedded are well separated in space from other structures. At the lower longitudes a weak extension toward Vela $\left(\ell \sim 270^{\circ}\right)$ can be seen-marked as Norma Wall extension. The latter is more pronounced at higher latitudes (see middle panel of Figure 16).

This Norma Wall is made up of various agglomerations. We will discuss them one by one, from high to low longitudes (left to right, respectively). Note that the galaxies around $6000 \mathrm{~km} \mathrm{~s}^{-1}$ and longitudes of $\sim 340^{\circ}-360^{\circ}$ are not part of the Norma Wall. They might form part of the outer boundary of 


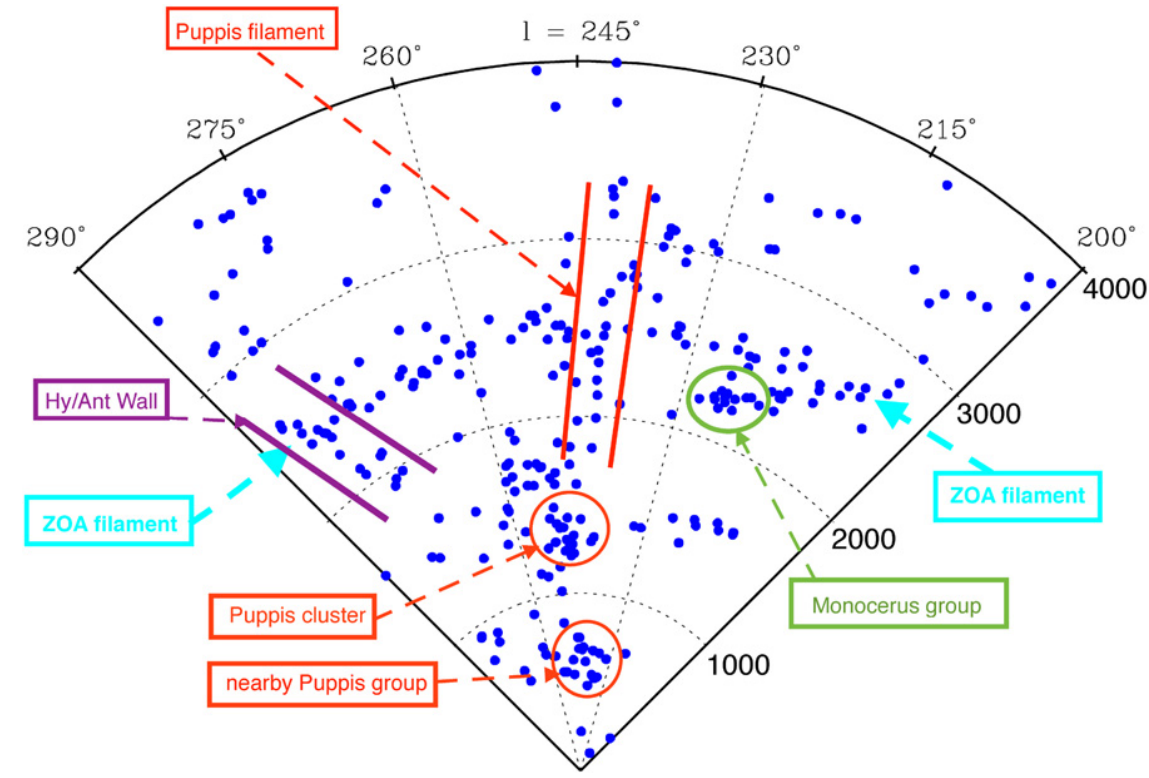

Figure 17. Zoomed-in wedge focusing on the crowded Puppis area $\left(290^{\circ}>\ell>200^{\circ} ;|b|<5.25 ; v_{\mathrm{LG}}<4000 \mathrm{~km} \mathrm{~s}^{-1}\right)$. The most prominent features mentioned in the text are marked. A very narrow filament or wall (marked "ZOA filament") can be traced at about $\sim 2500 \pm 100 \mathrm{~km} \mathrm{~s}^{-1}$ over the full longitude range of the wedge.

the LV though. The agglomeration around $340^{\circ}$, denoted as the 2nd Wall, is identified for the first time. It is marked with dashed lines because it is unclear whether this galaxy concentration extends above latitudes of $|b|>5^{\circ}$, owing to the overall high extinction and star density this close to the Galactic bulge. The on-sky distribution seems to hint at a further wall parallel to the Centaurus Wall but at higher longitudes (see middle panel of Figure 16). The Galactic bulge extension data, which will provide further $\mathrm{H}_{\mathrm{I}}$ detections up to higher latitudes around $(\ell, v) \sim\left(340^{\circ}, 4500 \mathrm{~km} \mathrm{~s}^{-1}\right)$, might shed further light on this. Nevertheless, this galaxy concentration is a further contributor to the mass overdensity in this part of the sky.

ZOA crossing of the Norma Wall.-The largest clump (marked in dark blue) contains galaxies that start at the outer fringes of the Norma Cluster, A3627 at $(\ell, b, v)=$ $\left(325^{\circ},-7^{\circ}, 4871 \mathrm{~km} \mathrm{~s}^{-1}\right.$ ) (Woudt et al. 2008), and extends diagonally across the ZOA (see also middle panel of Figure 16). A small cluster, marked as NW3, has been identified for the first time within the Norma Wall. NW3 lies deep in the ZOA at $(\ell, b, v)=\left(319^{\circ},-2^{\circ}, 4500 \mathrm{~km} \mathrm{~s}^{-1}\right)$. At higher positive latitudes, the Norma Wall then encompasses two further condensations, NW2 and NW1 $\left(\ell \sim 307^{\circ}\right.$ and $\left.300^{\circ}\right)$, which may be groups (or small clusters). None of these were previously known. Both are located at $b \sim 4^{\circ}$ and are quite distinct in Figure 16 (middle panel). In between these two groups, a short Finger of God is evident at slightly higher redshifts $(\ell, b, v)=\left(307^{\circ},+5^{\circ}, 5700 \mathrm{~km} \mathrm{~s}^{-1}\right)$. This is due to galaxies in the outskirts of the Centaurus-Crux/CIZA J1324.7 -5736 Cluster, discovered independently by (i) Fairall et al. (1998), Woudt \& Kraan-Korteweg (2001), and Woudt et al. (2004) from deep optical galaxy searches and systematic redshift follow-ups and (ii) Ebeling et al. (2002, 2005) and Mullis et al. (2005) in their search for X-ray clusters in the ZOA. This cluster is the second most massive cluster within the Norma Wall and has about $50 \%-70 \%$ of the mass of the Norma Cluster (Radburn-Smith et al. 2006). From the CIZA Cluster, the Norma Wall continues to higher latitudes and velocities (its lower-latitude part is still visible in the wedge), where it connects to the cluster in Vela, Abell S0639 at $(\ell, b, v)=\left(280^{\circ},+11^{\circ}, 6500 \mathrm{~km} \mathrm{~s}^{-1}\right)($ Stein 1997).

Centaurus Wall groups.-Apart from new groups and clusters that form part of the GA overdensity, other significant new structures are identified at lower redshifts. Notable is the cluster CW2 centered at about $\left(316^{\circ},+3^{\circ}, 3800 \mathrm{~km} \mathrm{~s}^{-1}\right)$, which is massive enough to display a small Finger of God. To the right of this (within the same red circle), a possible group of galaxies CW1 is visible at about $\left(309^{\circ}, 3.5,3400 \mathrm{~km} \mathrm{~s}^{-1}\right)$. They lie in front of the GA Wall and form part of the Centaurus Wall, a much narrower wall that lines up with the Centaurus Cluster $\left(302^{\circ}, 21^{\circ}, 3400 \mathrm{~km} \mathrm{~s}^{-1}\right)$. The clumps form part of the sine-wave feature (top panel of Figure 16). Two nearby filaments are also marked. They appear to point to the newly identified groups/clusters (marked by red lines), i.e., the one that approaches the cluster CW2 from the left at lower velocities is part of the sine-wave ZOA crossing visible in the on-sky projection at those longitudes $\left(315^{\circ}-\right.$ $330^{\circ}$ ). The other filament pointing toward the CW1 and CW2 clumps from the right (lower longitudes) is more difficult to discern in the on-sky distribution because it is cut into two parts by redshift division between the top and middle panels.

The PKS 1343-601 cluster.-What is not very prominent in the HIZOA detections is the cluster around the strong radio source PKS 1343-601, also known as Cen B. Because the presence of a strong radio source often is indicative of a massive cluster, there was previous speculation (KraanKorteweg \& Woudt 1999) whether a further dominant cluster that forms part of the Norma Supercluster could have escaped detection owing to its location at very low latitudes $\left(b=1{ }^{\circ} .7\right)$ and high extinction $\left(A_{\mathrm{B}}=10\right.$ m. 8; Schlafly \& Finkbeiner 2011). However, various deep NIR studies of the cluster (Nagayama et al. 2004, Schröder et al. 2007), as well as a careful investigation of its X-ray-flux by Ebeling et al. (2005), do not support this hypothesis. The PKS 1343-601 cluster seems to be an intermediate-size cluster and forms part of the GA overdensity. We find some galaxies in that general area and 


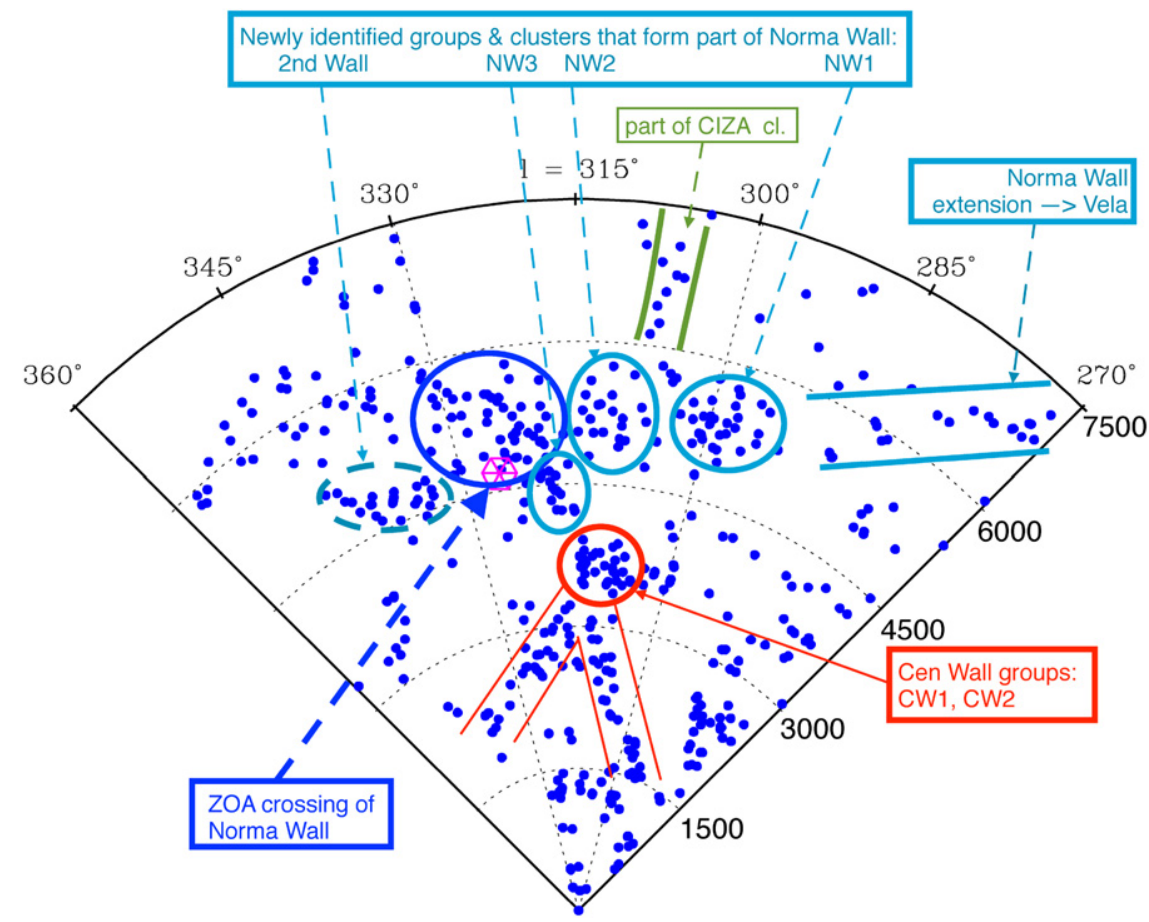

Figure 18. Zoomed-in wedge focused on the Norma Wall $\left(360^{\circ}>\ell>270^{\circ} ;|b|<5.25 ; \quad v_{\mathrm{LG}}<7500 \mathrm{~km} \mathrm{~s}^{-1}\right)$ showing HIZOA galaxies. Note the wall-like structure of the GA, the Norma Supercluster, at about $\sim 4800 \mathrm{~km} \mathrm{~s}^{-1}$ that stretches from about $340^{\circ} \gtrsim \ell \gtrsim 290^{\circ}$. The magenta hexagon marks the center of the Norma Cluster, which is located just below the HIZOA survey area $(\ell, b, v)=\left(325^{\circ},-7^{\circ}, 4871 \mathrm{~km} \mathrm{~s}^{-1}\right)$.

velocity, but not many. The strong radio continuum emission of Cen B (79 Jy at $1410 \mathrm{MHz}$; Wright \& Otrupcek 1990) suppresses galaxy detection in its immediate vicinity, but does not explain the absence of galaxies in our survey. It is possible that CW1, at only slightly higher latitude, is connected to or is part of this cluster.

Next to the Norma Cluster and the Centaurus-Crux Cluster, no further X-ray emission has been found that could point to the existence of a further massive cluster within the Norma Wall. However, X-rays are subject to photoelectric absorption by Galactic gas and confusion with Galactic sources, which limits detection close to the Galactic plane. Nevertheless, apart from the Norma and Centaurus Wall groups and clusters (NW1, NW2, NW3, CW1, and CW2), there is no evidence from the current $\mathrm{H}_{\mathrm{I}}$ survey, or previous surveys at other wavelengths, for further massive concentrations of galaxies within the Norma Wall.

\subsubsection{The LV Region}

The left third of the HIZOA redshift slice (Figure 15) is dominated by the LV. Both Figures 15 and 16 indicate that the LV underdensity appears to extend in the range of about $45^{\circ}>\ell>330^{\circ}$ for radial velocities $v_{\mathrm{LG}} \lesssim 6000 \mathrm{~km} \mathrm{~s}^{-1}$, consistent with Tully's early definition (Tully \& Fisher 1987). The left-hand boundary had been uncovered in HIZOA-N (Donley et al. 2005). The on-sky projections suggest that the $\mathrm{LV}$ is quite symmetric around the Galactic plane, reaching up to latitudes of about $\pm 40^{\circ}$ on either side. It is thus quite a formidable structure in the nearby universe. However, both the wedge and certainly the on-sky projection indicate that the LV is not nearly as empty as the previously available data suggest. The reason is that a large fraction of the $\mathrm{LV}$ is located behind the Galactic bulge, where dust extinction and star confusion reach much higher latitudes and the optical and NIR ZOAs are substantially wider. The current HIZOA data alone do not reach high enough Galactic latitudes to bridge the gap. It is for this reason that the above-mentioned Galactic bulge extension of HIZOA was launched. The Galactic bulge extension will improve the knowledge of the borders of the LV and Sagittarius Void $\left(350^{\circ},+0^{\circ}, 4500 \mathrm{~km} \mathrm{~s}^{-1}\right.$; Fairall 1998), as well as the Ophiuchus Cluster $\left(0.5,+9.3,9000 \mathrm{~km} \mathrm{~s}^{-1}\right)$ studied optically and spectroscopically by Hasegawa et al. (2000) and Wakamatsu et al. (2005).

The galaxies detected in the LV predominantly seem to be low-mass (gas-rich) dwarf galaxies. This was also confirmed in a preliminary analysis of the Galactic bulge extension (KraanKorteweg et al. 2008), which reveals some quite fine filaments, sparsely populated with low-mass galaxies, that permeate the LV. They probably are similar structures to what has been dubbed tendrils by Alpaslan et al. (2014) in the analysis of large-scale structures in GAMA. The LV population will be studied in more detail in a forthcoming paper.

\section{CONCLUSIONS}

We have presented data from a deep H i survey (HIZOA-S) with the multibeam receiver on the Parkes telescope in the southern "ZOA." The region covered $\left(36^{\circ}>\ell>212^{\circ}\right.$; $|b|<5^{\circ}$ ) includes the highest obscuration for optical/IR observations and, near the Galactic center, the highest stellar crowding. Of the 883 HIZOA-S galaxies detected, only $8 \%$ have existing optical redshifts. Nevertheless, on careful inspection of new and existing images and data sources, $78 \%$ of the detections have a counterpart visible in either the optical or the NIR wavebands. A third of these have not been previously cataloged. The median distance between the $\mathrm{HI}$ position and the counterpart is $1 ! 4$ (95\% of counterparts have 
Table 7

New Large-scale Structures Detected in the HIZOA Data

\begin{tabular}{|c|c|c|c|c|}
\hline Name & $\begin{array}{c}v_{\mathrm{LG}} \\
\left(\mathrm{km} \mathrm{s}^{-1}\right)\end{array}$ & $\ell$ & $b$ & Description \\
\hline Pup 1 group & $\sim 700$ & $\sim 245^{\circ}$ & $\sim+2.5$ & Loose group \\
\hline Pup 2 cluster & $\sim 1400$ & $\sim 248^{\circ}$ & $\sim+4^{\circ}$ & Dense group \\
\hline Pup 3 cluster & $\sim 6800$ & $\sim 242^{\circ}$ & $\sim 0^{\circ}$ & Cluster \\
\hline Monoceros group & $\sim 2200$ & $\sim 215^{\circ}-225^{\circ}$ & $\sim-3^{\circ}-+4^{\circ}$ & Elongated loose group \\
\hline ZOA filament & $2500 \pm 150$ & $\sim 210^{\circ}-285^{\circ}$ & $\sim \pm 4^{\circ}$ & Lengthy filament with small dispersion \\
\hline NW1 & $\sim 5600$ & $\sim 298^{\circ}$ & $\sim+4^{\circ}$ & NW galaxy concentration \\
\hline NW2 & $\sim 5200$ & $\sim 307^{\circ}$ & $\sim+4^{\circ}$ & NW galaxy concentration \\
\hline NW3 & $\sim 4500$ & $\sim 319^{\circ}$ & $\sim-2^{\circ}$ & New NW cluster \\
\hline 2nd Wall & $\sim 4800$ & $335^{\circ}-345^{\circ}$ & $\sim \pm 4^{\circ}$ & Possible new wall \\
\hline CW1 & $\sim 3400$ & $\sim 309^{\circ}$ & $\sim+3.5$ & New CW loose group \\
\hline CW2 & $\sim 3800$ & $\sim 316^{\circ}$ & $\sim+3^{\circ}$ & New CW cluster \\
\hline
\end{tabular}

$d<3 ! 8$ ), while the FWHP beam size is 15.5 . For detected galaxies with low $\mathrm{H}_{\mathrm{I}}$ masses that are in areas of high extinction, we have mostly been unable to find counterparts.

The survey (with an average rms of $6 \mathrm{mJy}$ ) is 2-3 times deeper than HIPASS (Meyer et al. 2004). To suppress ringing from strong Galactic $\mathrm{H}_{\mathrm{I}}$ and recombination line emission, spectra have been Hanning-smoothed, so the velocity resolution is $50 \%$ poorer $\left(27 \mathrm{~km} \mathrm{~s}^{-1}\right)$. For the 251 brighter galaxies in common, the derived $\mathrm{H}$ i parameters (velocity, width, and flux) for HIPASS and HIZOA-S are in excellent agreement.

The completeness as a function of Galactic background, flux, and velocity width for the HIZOA-S catalog has been characterized in a manner that is independent of assumptions of homogeneity. This allows HIZOA-S to be used for quantitative study of the ZOA density field. The flux completeness limit for the main survey region (excluding edges) and at Galactic continuum temperatures $<7 \mathrm{~K}$ is $2.8 \mathrm{Jy} \mathrm{km} \mathrm{s}^{-1}$ at the mean sample velocity width of $160 \mathrm{~km} \mathrm{~s}^{-1}$. This corresponds to a mean flux density limit of $17 \mathrm{mJy}$. The flux integral and flux density completeness limits scale as $w^{0.74}$ and $w^{-0.26}$, respectively; i.e., galaxies with narrow widths can be found at lower flux integrals and higher mean flux densities. There is some incompleteness at higher fluxes, but we also find that $9 \%$ of HIZOA galaxies are detected at lower fluxes.

Several interesting new objects have been noted in HIZOA data and already published, including the nearby extended objects HIZOA J1514-52, J1532-56, and J1616-55 (Staveley-Smith et al. 1998), the new nearby galaxy HIZSS 003 (Henning et al. 2000, Massey et al. 2003; Begum et al. 2005), the fast rotator J1416-58 (Juraszek et al. 2000), and the massive galaxy J0836-43 (Donley et al. 2006; Cluver et al. 2008, 2010). Notably, no new nearby, massive galaxies similar to Circinus have been discovered, and there is no further room in the ZOA for such an object to be hidden within the areas mapped so far. However, the new catalog does contain a new galaxy, HIZOA J1353-58, which seems to be a possible companion (and the only candidate found to date) for the Circinus galaxy.

The main new results identified in this paper are the groups and clusters discussed in Section 7. For clarity, these are summarized in Table 7 . The HIZOA survey has clearly proved to be a highly successful approach in uncovering the large-scale structures in the ZOA, most of which were previously unknown. This is particularly striking in the redshift cone in Figure 15, which reveals large-scale structure within the deepest obscuration layer of the Milky Way, reminiscent of the redshift cone of the Coma Cluster in the so-called Great Wall (de Lapparent et al. 1986). The main difference is that the main Norma Cluster lies just beyond the borders of the HIZOA survey. The combination with known structures adjacent to the ZOA (Figure 16) shows how many of the $\mathrm{H}$ I-detected features link up. HIZOA has resulted in an improved census of the GA region and an improved understanding of the extent of the Norma Supercluster. The diagonal crossing of the supercluster is well traced by the new data and has led to the identification of further galaxy concentrations that form part of the wall-like structure, such as the NW3 cluster deep in the plane and the NW2 and NW1 complexes that connect to the Cen-Crux clusters that lie just at the border of this survey. Hence, the Norma Wall is now seen to extend from Pavo II to the Norma Cluster, the NW3 cluster, the NW2, NW1, and the CIZA clusters to the Vela Cluster. Also remarkable are the two nearer clusters that lie within the Centaurus Wall, which have filaments pointing toward them, very similar to the legs and body of the Great Wall "stickman." We have also newly identified a potential second crossing at higher latitudes. Although not part of the Norma Supercluster despite its redshift, this galaxy concentration also adds to the overall mass of the GA overdensity.

In the Puppis region, some of the unveiled clusters and groups have been seen in earlier $\mathrm{H}_{\mathrm{I}}$ observations. However, what is confirmed is the earlier suspected Hy/Ant Wall, which can now be traced across the ZOA, as well as a full mapping of the Puppis filament, which forms part of the major sine-wave structure seen in the top panel of Figure 16. A new discovery is the Monoceros group and a filamentary feature at mostly constant redshift that runs within the $\mathrm{ZOA}$ for a longitude range of nearly $80^{\circ}$.

HIZOA has also confirmed the large extent of the LV. Nevertheless, quite a few nearby galaxies have been found behind the Galactic bulge, indicating that the void is not quite as empty as previously suggested.

These results bode well for the future with the forthcoming H I surveys that are planned with the SKA Pathfinders ASKAP (Wallaby) and WSRT/APERTIF. These surveys will together cover the whole sky and provide for the first time a deeper $(z=0-0.26)$ and well-resolved complete census of the largescale structures in the sky, inclusive of the Milky Way.

The Parkes radio telescope is part of the Australia Telescope National Facility, which is funded by the Commonwealth of 
Australia for operation as a National Facility managed by CSIRO. We would like to thank the staff at the Parkes Observatory for all their support, D. Barnes, M. R. Calabretta, R. F. Haynes, A. J. Green, S. Juraszek, M. Kesteven, S. Mader, R. M. Price, and E. M. Sadler for their assistance during the course of the survey, and K. Said for early access to the IRSF imaging data of the HIZOA galaxies, as well as his help with some of the data reduction.

Parts of this research were conducted by the Australian Research Council Centre of Excellence for All-sky Astrophysics (CAASTRO), through project number CE110001020. R.K.K. and A.C.S. acknowledge the research support they received from the South African National Research Foundation. P.A.H. thanks the NSF for support for the early stages of this work through the NSF Faculty Early Career Development (CAREER) Program award AST 95-02268.

This research has made use of the NASA/IPAC Extragalactic Database (NED), which is operated by the Jet Propulsion Laboratory, California Institute of Technology, under contract with the National Aeronautics and Space Administration; the Digitized Sky Surveys, which were produced at the Space Telescope Science Institute under U.S. Government grant NAG W-2166; the Sloan Digital Sky Survey, which is managed by the Astrophysical Research Consortium for the Participating Institutions; the HIPASS data archive, provided by the ATNF under the auspices of the Multibeam Survey Working Group; the SIMBAD database, operated at CDS, Strasbourg, France, and of the SuperCOSMOS Sky Surveys, the WFCAM and VISTA Science Archives, operated at the Royal Observatory of Edinburgh (WFAU); the HyperLEDA database (http://leda.univ-lyon1. fr); data products from the Two Micron All Sky Survey, which is a joint project of the University of Massachusetts and the Infrared Processing and Analysis Center, funded by the National Aeronautics and Space Administration and the National Science Foundation; the NASA/IPAC Infrared Science Archive, which is operated by the Jet Propulsion Laboratory, California Institute of Technology, under contract with the National Aeronautics and Space Administration.

\section{APPENDIX}

\section{NOTES TO INDIVIDUAL DETECTIONS}

J0631-01.- The distance between the $\mathrm{H}$ i position and the counterpart is rather large $\left(d_{\text {sep }}=6 ! 1\right)$, but the $\mathrm{H}_{\mathrm{I}}$ detection is located near the corner of the cube $\left(l, b=212^{\circ} .2,-5^{\circ} .1\right)$, where the sensitivity is lower. On the other hand, the HIPASS position is much closer to the counterpart $\left(d_{\text {sep }}=2 ! 3\right)$.

J0647-00A.- There is a possible galaxy visible in the NIR next to a group of stars at (R.A., decl.) $=(06: 47: 06.0$, $-00: 35: 27)$, with a distance of 1.2 to the $\mathrm{H}$ I position.

J0653-03A, J0653-03B, and J0653-04.-The spectra of all three detections show emission from the other galaxies as well.

J0657-05A and J0657-05B.-Both spectra show emission of the other galaxy as well.

J0700-04.-Begum et al. (2005) observed this galaxy with the VLA and found that the $\mathrm{H}$ i emission comes from two dwarf galaxies. Both are faintly visible on the deep NIR images.

J0704-13.- Underneath the narrow H i profile there appears to be a broader profile (under the detection threshold) at a more northern decl., as can be made out in the data cube. The obvious cross-match for the narrow profile is the nearly face-on galaxy 2MASX J07042532-1346257, while farther north (at about equal distance from the fitted $\mathrm{H}$ i position) lies a galaxy pair, 2MASX J07041909-1338217 and an unpublished galaxy at (R.A., decl. $)=(07: 04: 18.7,-13: 37: 47)$, which is likely the counterpart of the broader profile.

J0709-03.-This galaxy is not in NED but was found in SIMBAD.

J0717-22B and J0717-22A.- These two detections are close together, and the images show a close galaxy pair that is likely interacting (it is bright in IRAS as well as WISE). There is a (single) optical velocity for the IRAS source $\left(2750 \pm 70 \mathrm{~km} \mathrm{~s}^{-1}\right.$; Visvanathan \& Yamada 1996) that agrees well with the main $\mathrm{H}_{\mathrm{I}}$ detection (J0717-22A). There are also three smaller galaxies in the area, two of which are likely background, but one (2MASX J07174038-2224406) is a possible candidate, too. The $\mathrm{H}$ I detection J0717-22A comes most likely either from the more edge-on component of the galaxy pair (CGMW 2-0730) or from both (the profile is lopsided). The narrow profile J0717-22B could come from the less inclined component of the galaxy pair (CGMW 2-0731), or it could come from a hidden late-type galaxy $\left(A_{\mathrm{B}}=3 \mathrm{~m} .8\right)$. It is less likely coming from 2MASX J07174038-2224406, which seems nearly edge-on (though at these extinction levels faint outer spiral arms could be invisible).

J0718-09.- There are two low surface brightness (LSB) galaxies in the field; the closer and more extended one is given as the cross-match. It is possible, though, that the other galaxy at $($ R.A., decl. $)=(07: 18: 14.5,-09: 03: 03)$ may contribute to the signal (see J0700-04).

J0725-24A.-The profile shows a high-velocity shoulder. A close inspection of the data cube indicates a fainter $\mathrm{H}$ i emission at smaller R.A. with the obvious candidate 2MASX J07245535-2430057.

J0732-16. - This seems to be a star-forming region with an $\mathrm{H}$ II region and star cluster. There is a $2 \mathrm{MASS}$ detection nearby (2MASX J07315300-1658237) that looks like a small, nearly edge-on galaxy. However, the extinction in this area is large and spatially highly variable, which means that the galaxy could be also be a nearly face-on barred spiral. Owing to the large spatial variation of the extinction, a hidden galaxy is also possible.

J0733-28. - The profile shows a high-velocity shoulder that seems to be caused by the inclined galaxy at (R.A., decl. $)=(07: 33: 23.2,-28: 44: 01)$.

0738-26. - A galaxy pair is the obvious match for the $\mathrm{HI}$ detection. Both galaxies seem to be of similar (late) type, with CGMW 2-1056 being more edge-on and CGMW 2-1059 being less inclined. It is likely that both have $\mathrm{H}$ I and possibly contribute to the $\mathrm{H}$ i detection.

J0742-20A.- There are at least five galaxies of similar size visible that could form a group of galaxies. The counterpart is a large spiral that is the most prominent in the $B$ band. It is also the reddest on WISE images.

J0743-32.- There are two 2MASS galaxies in the area, but both are of earlier morphological type than the cross-match and hence less likely candidates. Nevertheless, they cannot be fully excluded as candidates.

J0744-26 and J0744-25.- These two H I detections are only 2.5 apart. There are five galaxies visible, two of which are possible candidates. 2MASX J07442148-2602486 is the larger candidate and a late-type spiral. It agrees well with the profile of J0744-26, while the other galaxy, 2MASX J07442386-2600016, seems to be an early-type spiral with a 
smaller diameter, which agrees better with J0744-25. Paturel et al. (2003) observed 2MASX J07442386-2600016 and found a detection at $v=4686 \mathrm{~km} \mathrm{~s}^{-1}$ though with a very low $\mathrm{S} / \mathrm{N}$ of 2.7 .

J0745-18 and J0746-18.-Both spectra show emission of the other galaxy as well.

J0747-21.-2MASX J07471872-2138080 is confirmed through an optical velocity of $v=6988 \pm 40 \mathrm{~km} \mathrm{~s}^{-1}$ (Visvanathan \& Yamada 1996). A nearby early spiral could be a companion.

J0748-25A and J0748-25B.-These two $\mathrm{H}$ I detections are 4! 1 apart (and at different velocities). There are six possible candidates in the area. 2MASXJ07483666-2510211 was detected by Paturel et al. (2003) at $v=6809 \mathrm{~km} \mathrm{~s}^{-1}$, while Kraan-Korteweg et al. (2015, in preparation) have observed 2MASXJ07483252-2516431 at $v=6804 \mathrm{~km} \mathrm{~s}^{-1}$. Both used the Nançay Radio Telescope (NRT) and have measured a similar flux ( $\sim .5 \mathrm{Jy} \mathrm{km} \mathrm{s}^{-1}$ and $\sim 5.1 \mathrm{Jy} \mathrm{km} \mathrm{s}^{-1}$, respectively), while our detection has a higher flux. We therefore conclude that 2MASXJ07483452-2513191, which is closer to our $\mathrm{HI}$ position, is the counterpart of J0748-25B.

Owing to the two NRT observations, we can exclude four of the six galaxies as the candidate for J0748-25A. 2MASX J07480561-2513459 has an optical velocity of $v=7980 \mathrm{~km} \mathrm{~s}^{-1}$ (Acker et al. 1991) and is an IRAS galaxy. It is the most likely candidate for $\mathrm{J} 0748-25 \mathrm{~A}$.

J0748-26, J0749-26A, and J0749-26B.-These three H I detections are close together, and the profiles are confused. While J0748-26 and J0749-26B have obvious candidates, MIRIAD failed to fit the position of J0749-26A, and the distance to the cross-match could be larger. There are two candidates, ESO 493-G017 and 2MASX J07490476-2611061. ESO 493-G017 is an Sab galaxy and was observed with the NRT by Chamaraux et al. (1999), who find a lower peak flux ( 60 mJy). 2MASX J07490476-2611061 is therefore the more likely counterpart.

J0751-26.-A possible candidate looks small and seems to be a more distant galaxy. However, an LSB halo cannot be excluded (WISE images show considerable [foreground] emission in the W3 and W4 bands and are therefore not conclusive).

J0753-22.-The candidate is a galaxy triple (IRAS source), and if they are companions, the $\mathrm{H}_{\mathrm{I}}$ signal could come from more than one of them. Note that the $\mathrm{H}_{\mathrm{I}}$ detection in the literature is associated with CGMW 2-1471, which is the smallest galaxy in the triplet. There are more galaxies in the area that may indicate a galaxy group.

J0755-23.-At least five candidates are visible in the area. The largest and brightest, 2MASX J07551159-2304175, is an inclined early spiral and a likely candidate for this $\mathrm{H}$ I mass $\left(\log M_{\mathrm{H}}=9.8\right)$. Two other galaxies, CGMW 2-1571 and a new galaxy at (R.A., decl.) $=(07: 55: 34.1,-23: 04: 33)$, are also large but bluer and of later morphological type. This is possibly a group of galaxies.

J0756-26.-The profile is confused. The HIPASS profile, with a position $d_{\text {sep }}=5^{\prime}$ farther south, extends only to $v \sim 6700 \mathrm{~km} \mathrm{~s}^{-1}$, while our profile extends to $v \sim 6800 \mathrm{~km} \mathrm{~s}^{-1}$. Using the HIPASS webpage, the position of our candidate gives a more similar profile to ours. The HIZOA cube shows a peak at (R.A., decl.) $\simeq$ (07:56:47, -26:16:27), which was not identified as a separate detection during the search. We conclude that the confusing partner is 2MASX J07563846-2615018 far in the south. Huchtmeier et al. (2001) observed this galaxy with the
Effelsberg Radio Telescope at an $\mathrm{H}$ i velocity of $241 \mathrm{~km} \mathrm{~s}^{-1}$. The HIZOA data cube does not show anything at this velocity and with a peak flux of $67 \mathrm{mJy}$ and a width of $w_{50}=26 \mathrm{~km} \mathrm{~s}^{-1}$.

J0756-31.- There are two equally sized galaxies close together, possibly a pair. One seems edge-on; the other has a small inclination. The profile is more consistent with the less inclined galaxy.

J0802-22.-The counterpart, ESO 494-G013, was also observed with the Effelsberg Radio Telescope by Kraan-Korteweg \& Huchtmeier (1992) and detected at $v=1983 \mathrm{~km} \mathrm{~s}^{-1}$. We could not confirm this detection at the given peak flux of $\sim 0.15 \mathrm{Jy}$.

J0805-27 and J0806-27.- These two Hi detections are close together, and the profiles cannot be fully separated, that is, some of the H i parameters are uncertain.

J0807-25.-Kraan-Korteweg \& Huchtmeier (1992) detected this galaxy with the Effelsberg Radio Telescope at $v=1019 \mathrm{~km} \mathrm{~s}^{-1}$, while our detection is at $v=7679 \mathrm{~km} \mathrm{~s}^{-1}$. The HIPASS archive ${ }^{24}$ does not show any (bright) detection at this position and velocity.

J0808-35.- There are two candidates of similar size: 2MASX J08083663-3558328 is of a later spiral type and more inclined than 2MASX J08080066-3558243. The H I detection lies between these two galaxies, and from a closer inspection of the data cube it is likely that both galaxies contribute to the signal. C. Kraan-Korteweg et al. (2016, in preparation), using the NRT, detected 2MASX J08080066-3558243 without the broader base of our detection (which is therefore likely due to 2MASX J08083663-3558328).

J0816-27B.-A diffuse emission visible in the $B$ band at $(\mathrm{R}$. A., decl. $)=(08: 17: 07.0,-27: 45: 15)$ at a distance of $3 ! .0$ to the $\mathrm{H}_{\mathrm{I}}$ position is the likely counterpart if confirmed as a galaxy.

J0817-27.-The H I profile seems to be either disturbed or confused. The counterpart is NGC 2559, which appears also disturbed in the optical (Corwin et al. 1985). There is an LSB dwarf close by, at (R.A., decl.) $=(08: 17: 24.4,-27: 28: 04)$, which may be a companion. At about $18^{\prime}$ north and south are two other H I detections (J0816-27A and J0816-27B) with similar velocities.

J0817-29A.-This cross-match is listed in SIMBAD but not in NED.

J0822-36.-There is a possible galaxy visible in the NIR at (R.A., decl. $)=(08: 22: 50.4,-36: 36: 41)$, with a distance of 0.5 to the $\mathrm{H}_{\mathrm{I}}$ position, which, if real, is a likely counterpart.

J0824-41.-There are two similar-looking galaxies, possibly a galaxy pair. 2MASX J08240445-4144221 is more inclined, while 2MASX J08235945-4143111 seems to show a bar and the start of a spiral arm; both match the profile well enough. The profile has a low $\mathrm{S} / \mathrm{N}$ of 5, and it is not possible to tell whether one or both galaxies contribute to the signal.

J0827-35.-Paturel et al. (2003) have observed IRAS 08254-3512, which is 5:4 north of our detection. Their detection is similar to ours with a slightly lower peak flux. Nothing is visible at the IRAS position, and the counterpart is more likely an invisible LSB between these two positions (which would agree with the narrow single-peak profile and low $\mathrm{H}_{\mathrm{I}}$ mass $\left(\log M_{\mathrm{H} \mathrm{I}}=8.0\right)$.

J0827-36.- There are two galaxies close together, probably a galaxy pair. 2MASX J08271103-3629389 is edge-on and seems to match the profile better; it also has an optical velocity

\footnotetext{
${ }^{24}$ http://www.atnf.csiro.au/research/multibeam/release/
} 
of $9512 \pm 40 \mathrm{~km} \mathrm{~s}^{-1}$ (Visvanathan \& Yamada 1996). The pair was detected by IRAS as well.

J0834-37. - The cross-match is a nearly face-on spiral galaxy. There is a diffuse LSB galaxy visible in the $B$ band close by, at (R.A., decl.) $=(08: 34: 16.9,-37: 32: 36)$, which may contribute to the signal.

J0851-44.- There are two possible counterparts visible in the NIR, one edge-on and one a little inclined. The profile seems to match the galaxy with less inclination better.

J0857-39 and J0858-39.-Both spectra show emission of the other galaxy as well.

J0858-45A.- There are two possible counterparts visible in the NIR, possibly a galaxy pair. 2MASX J08580941-4548126 shows spiral arms, either disturbed or with a companion close by, while 2MASX J08581399-4550547 is a very inclined early-type spiral. The profile matches the former slightly better, and a possible contribution from both galaxies cannot be excluded.

J0902-53.- There is a small edge-on galaxy in the field. Even taking the extinction into account $\left(A_{\mathrm{B}}=2 \mathrm{~m} .7\right)$, the galaxy seems too small for this distance $\left(v=7122 \mathrm{~km} \mathrm{~s}^{-1}\right)$ and $\mathrm{H}_{\mathrm{I}}$ mass $\left(\log M_{\mathrm{H} \text { I }}=9.8\right)$ but cannot be fully excluded.

J0916-54A and J0916-54B.-Both spectra show emission of the other galaxy as well.

J0927-48. - The high-velocity shoulder in the profile is likely caused by J0929-48.

J0932-51.- The cross-match is an interacting galaxy pair (IRAS source). Both are spirals, and it is not possible to decide where the $\mathrm{H}$ i comes from.

J0939-56.- There is a possible galaxy visible in the NIR at (R.A., decl. $)=(09: 39: 36.3,-56: 53: 05)$ with a distance of 0 '.8 to the $\mathrm{H}_{\mathrm{I}}$ position, which, if real, matches the profile well.

J0949-47A.- The cross-match (ESO 213-G002) is a peculiar S0 galaxy that seems to have either a companion or a jet to the south. Farther south $\left(d_{\text {sep }}=3 ! 6\right)$ is RKK 1666, which is likely to contribute: the data cube shows a faint, narrower profile at (R.A., decl.) $\simeq$ (09:49:24, -47:59:48).

J0949-56.- The distance between the $\mathrm{H}$ I position and the position of the cross-match, $d_{\text {sep }}=11$ ! 8 , is for an $\mathrm{S} / \mathrm{N}$ of 77 rather large. A closer inspection of the data cube revealed a fainter $\mathrm{H}_{\text {I }}$ detection close by $\left(d_{\mathrm{sep}}=1\right.$.6 $)$ with an approximate position of $($ R.A., decl.) $\simeq(09: 48: 18.6,-56: 29: 49)$ and $v \simeq 1641 \mathrm{~km} \mathrm{~s}^{-1}$, which appears as a low-velocity shoulder in the profile of J0949-56. The obvious candidate here is 2MASX J09482319-5627370.

J0950-49. - There are two different optical velocities for this galaxy: $v=12391 \pm 500 \mathrm{~km} \mathrm{~s}^{-1} \quad$ (Kraan-Korteweg et al. 1995) is uncertain and seems high for a galaxy of this appearance; $v=4043 \pm 82 \mathrm{~km} \mathrm{~s}^{-1}$ (Saunders et al. 2000) is about $3 \sigma$ from our $\mathrm{HI}_{\mathrm{I}}$ velocity and agrees better with the optical appearance of the galaxy.

J0950-52.-A possible candidate visible at all wavelengths is close to a star, which makes it difficult to determine its appearance, but it seems too small for this velocity $\left(v=2244 \mathrm{~km} \mathrm{~s}^{-1}\right)$. The star and the extinction $\left(A_{\mathrm{B}}=4.3\right)$ could hide a larger halo, but a hidden LSB galaxy is a more likely cross-match. The galaxy is a possible IRAS source (IRAS 09482-5209; though it could be also the bright star to the north).

J0952-61.- No galaxy is visible at the position of RKK 1733 . It is only $d_{\text {sep }}=0$ '. 8 from the 2MASS position, which is not listed by Kraan-Korteweg (2000), though it is clearly visible in the $B$ band. It seems possible that both IDs indicate the same galaxy.

J0952-55A.- The profile is highly lopsided. The crossmatch is large and diffuse with a small bulge in the NIR. It is not possible to distinguish whether it may be disturbed or may have a small companion.

J0953-49.- There is a large LSB galaxy visible in the $B$ band, as well as a smaller inclined galaxy (RKK 1732). The LSB galaxy matches the profile better.

J1000-58A and J1000-58B.-The two counterparts are only 1.5 apart, and the two profiles cannot be fully separated.

J1004-58.- The profile shows a prominent horn that, according to the data cube, could be caused by two close-by double-horn profiles separated in both R.A. and decl. (10:04:34, $-58: 31: 10$ and 10:04:04, $-58: 39: 15$, respectively). There are two NIR-bright candidates in these positions, which are likely counterparts. This is supported by that fact that the distances between $\mathrm{HI}$ position and the two counterparts $\left(d_{\text {sep }}=4.8\right.$ and $\left.d_{\text {sep }}=4 ! 2\right)$ are large for an $\mathrm{S} / \mathrm{N}$ of 36 ; see also Figure 4.

J1024-52.- The candidate seems to be a galaxy pair (possibly interacting since it is an IRAS source). 2MASX J10240866-5159040 is slightly rounder and of earlier type than RKK 2457, and it is not quite clear whether only one of them or both contribute to the signal.

J1058-66.-This is a confused profile. the HIPASS detection J1059-66 lies farther south and shows a broad double-horn profile clearly caused by ESO 093-G003 with an optical measurement of $v=1470 \pm 270 \mathrm{~km} \mathrm{~s}^{-1}$ (Fairall 1980). Our $\mathrm{H}$ I detection could not be separated from this because the ESO galaxy lies beyond the edge of the cube and was therefore not properly detected.

J1149-64.- - There is a large spiral with a smaller companion visible in the NIR. It is likely that the companion contributes to the profile.

J1214-58.-There are two galaxies in the field, possibly companions. WKK 0788 is edge-on and seems to match the profile slightly better than the later type WKK 0782. There is no indication of confusion.

J1222-57.- Schröder et al. (2009) have detected this galaxy in the OFF beam and associated it with WKK 0969 (which lies $d_{\text {sep }}=10$ '. 2 to the southeast of our detection) as the only known galaxy in the area, while at $d_{\text {sep }}=6$ ! 2 we find a galaxy that is not listed in the literature as the more likely counterpart.

J1234-61.- There are two galaxies visible, possibly a galaxy pair. They have similar appearance except that the galaxy at (R.A., decl.) $=(12: 33: 47.8,-61: 29: 57)$ appears more face-on. The profile appears to be confused, and it is likely that both galaxies are detected.

J1304-58. - The distance of 5'.6 to the cross-match is large, but the $\mathrm{H}$ i detection is near the edge of the cube and the $\mathrm{S} / \mathrm{N}$ is low. The galaxy is well visible in the $B$ band. The other candidates in the field do not agree as well with the profile.

J1310-57.- There are two bright galaxies (E and S) in the field and several smaller ones, possibly a group of galaxies.

J1314-58.- This is a strong signal with a lopsided double horn. There are two galaxies in the field: WKK 2020 is more edge-on and a later spiral than 2MASX J13150989-5856116, which has an optical velocity of $v=2355 \pm 50 \mathrm{~km} \mathrm{~s}^{-1}$ (Fairall 1998). They are likely to form a pair with both contributing to the signal. This is supported by the fact that the distance to each of the galaxies $\left(d_{\text {sep }}=2.5\right.$ and $d_{\text {sep }}=3 ! .0$, 
respectively) is unusually high for the $\mathrm{S} / \mathrm{N}$ of 96 of the $\mathrm{HI}$ detection: the median $\mathrm{S} / \mathrm{N}$ of cross-matches with distances between $2 ! .0$ and 3.0 is 9.8 with a sigma of 7.2 ; hence, this is a $12 \sigma$ deviation. In addition, the $\mathrm{H}_{\mathrm{I}}$ position lies just between these two galaxies.

J1329-61.- There is a possible galaxy visible in the NIR at (R.A., decl. $)=(13: 30: 01.0,-61: 51: 44)$ with a distance of 1 !.2 to the $\mathrm{H}$ I position, which matches the profile well.

J1337-58B.-There are three spiral galaxies in the field, two of which are of similar size and possibly form a pair. 2MASX J13372458-5852216 is fairly edge-on and of earlier morphological type than 2MASX J13373282-5854136, which has an optical velocity of $v=3709 \pm 70 \mathrm{~km} \mathrm{~s}^{-1}$ (Visvanathan \& Yamada 1996). It seems likely that both galaxies contribute to the profile (see, for example, the profile given in Schröder et al. [2009] for WKK $2390=2$ MASX J13372458-5852216).

J1344-65.-The counterpart, WKK 2503, was also observed and detected by Schröder et al. (2009). Their profile, with a higher velocity resolution, is clearly confused, that is, the peak at $2750-2950 \mathrm{~km} \mathrm{~s}^{-1}$ seems to belong to a second galaxy that is visible neither in the optical nor in the NIR.

J1353-58.- This $\mathrm{H}_{\mathrm{I}}$ detection seems to be a companion of the Circinus galaxy (J1413-65). We have adopted the same distance to calculate the $\mathrm{H}$ i mass.

J1405-65.- There are two LSB galaxies in the field. The fainter and rounder seems to fit the profile better. The cube shows a possible faint $\mathrm{H}$ i detection under the detection limit at $v \simeq 3400 \mathrm{~km} \mathrm{~s}^{-1}$ and with a larger line width, which could be the other, more edge-on, galaxy in the field (WKK 2924). WKK 2924 was also observed by Schröder et al. (2009), where both detections were noted. While the detection at $3410 \mathrm{~km} \mathrm{~s}^{-1}$ was attributed to WKK 2924 (as we do), the detection at $2864 \mathrm{~km} \mathrm{~s}^{-1}$ was attributed to WKK 2938, which lies to the south at $d_{\text {sep }}=7 ! 2$. However, the position and flux of our $\mathrm{H} \mathrm{I}_{\mathrm{I}}$ detection rule out WKK 2938 as a counterpart.

J1406-57.- There is a galaxy pair in the field. Their morphological types seem to be similar, and 2MASX J14061018-5752098 is edge-on while 2MASX J140627395751425 is less inclined. It is likely that both contribute to the profile (the data cube seems to support a separation in R.A.).

J1413-65.-This is the well-known Circinus galaxy. It is resolved with respect to the Parkes beam.

J1416-58.-This galaxy has the largest line width: $w_{50}=699 \pm 12 \mathrm{~km} \mathrm{~s}^{-1}$ (and $w_{20}=730 \pm 18 \mathrm{~km} \mathrm{~s}^{-1}$ ). The NIR images show a perfect edge-on galaxy with a clear bulge.

J1424-60.-There is a possible galaxy visible in the NIR at (R.A., decl. $)=(14: 25: 10.3,-60: 05: 49)$ with a distance of 2.5 to the $\mathrm{H}$ i position, which matches the profile well. However, owing to the high extinction and faintness of the object, we cannot say for certain that this is a galaxy.

J1435-61.-There are several galaxies visible on the VISTA image. Owing to the high extinction $\left(A_{\mathrm{B}}=26^{\mathrm{m}} \cdot 6\right)$, only the bulges are clearly visible, and it is not possible to compare sizes and morphological types. We have chosen the largest as the most likely counterpart, which is also red on WISE.

J1448-54.-The H I detection is a nearby dwarf galaxy $(D=11.5 \mathrm{Mpc})$. The candidate appears very small, but with an extinction of $A_{\mathrm{B}}=3.3$ a large LSB halo is possible.

J1452-56. - The cross-match is a very edge-on late-type spiral galaxy. It seems to have a companion to the north that is less inclined but of similar morphological type. According to the data cube, it is likely that both contribute to the profile.
J1501-57.- There is a galaxy pair in the field, both very similar-looking inclined medium-type spirals. The profile, however, implies a galaxy with little inclination. A closer inspection of the data cube reveals a broader profile under the detection limit, similar to J1004-58. On the other hand, the extinction in this region is fairly high $\left(A_{\mathrm{B}}=10^{\mathrm{m}} .5\right)$ and varies across the field (which is most prominent in the $I$ band). Therefore, either the cross-match is an invisible face-on/latetype galaxy, or we have detected a lopsided horn of one of the galaxies in the pair (note that the baseline is very variable here).

J1504-55.- There is a possible galaxy visible in the NIR at (R.A., decl. $)=(15: 04: 24.3,-55: 29: 09)$ with a distance of 1.2 to the $\mathrm{H}_{\mathrm{I}}$ position. It lies between stars, and this cannot be unambiguously identified as a galaxy.

J1509-52.-This H i detection is resolved with respect to the Parkes beam.

J1513-54.- This is a close galaxy pair, possibly interacting (IRAS source). The larger component is listed here.

J1514-52.-This H I detection is resolved with respect to the Parkes beam.

J1530-54.- There is a possible galaxy visible in the NIR at (R.A., decl. $)=(15: 31: 00.5,-54: 03: 37)$ with a distance of 0.2 to the $\mathrm{H}$ i position, which matches the profile well.

J1530-59. - The cross-match is a close galaxy pair, possibly interacting (IRAS source). It is not possible to tell whether one or both contribute to the profile.

J1532-56.- This galaxy is extended and has been observed with the Australia Telescope Compact Array by StaveleySmith et al. (1998) with a positional accuracy of $15^{\prime \prime}$ (NED). At an extinction of $A_{\mathrm{B}}=57^{\mathrm{m}}$ nothing is visible at this position.

J1549-60.-There are three galaxies in the field. The counterpart seems slightly larger in the $B$ band, as well as with WISE, than WKK 5337 and the third galaxy at (R.A., decl. $)=(15: 49: 39.6,-60: 15: 24)$.

J1550-58.- Schröder et al. (2009) observed WKK 5366 (at $d_{\text {sep }}=4$.9) with almost the same flux. We have excluded WKK 5366 as a counterpart since it has an optical velocity of $4822 \pm 82 \mathrm{~km} \mathrm{~s}^{-1}$ (Woudt et al. 2004), and the distance is fairly large for such a bright detection.

J1553-50.-Despite the high extinction $\left(A_{\mathrm{B}}=9^{\mathrm{m}} .2\right)$, several small galaxies of similar appearance can be seen, possibly forming a group. We have chosen the largest with obvious bulge and disk as the probable counterpart.

J1553-49 and J1554-50.- This is an area of complex emission that cannot easily be disentangled. The data cube shows also some fainter emission below our detection limit. While the cross-match for J1554-50 is a nearly edge-on galaxy with no other galaxy visible within a $5^{\prime}$ radius, for $\mathrm{J} 1553$ -49 there are four galaxies visible in the NIR (two of which are unknown). The largest, 2MASX J15534458-5000182, seems to be an early-type galaxy and is an unlikely candidate. The galaxy at (R.A., decl.) $=(15: 54: 01.1,-49: 54: 06)$ shows a diffuse halo and a small bulge and seems to be the most likely candidate. It is also bright in the WISE bands W3 and W4. A candidate for possible confusion could be 2MASX $\mathrm{J} 15534427-5002132$ at 4.8 from the $\mathrm{H}$ I position.

J1553-61.-Schröder et al. (2009) observed WKK 5430, which is 5.3 to the north of our position. Their detection is lopsided and has less flux than ours, which rules out WKK 5430 as the counterpart. In addition, the HIPASS position (R.A., decl.) $=(15: 53: 43.5, \quad-61: 13: 34)$ favors WKK 5447. 
J1557-50A and J1557-50B.-These two H detections are 6.5 apart, and the two profiles are slightly confused. The extinction is fairly high $\left(A_{\mathrm{B}} \simeq 17^{\mathrm{m}}\right)$, and a hidden galaxy cannot be excluded.

J1603-49.- There are two candidates, both nearly edge-on. They are of similar size, possibly a galaxy pair. The candidate at (R.A., decl.) $=(16: 03: 34.6,-49: 51: 16)$ is slightly larger with a larger bulge (it is also brighter on WISE).

J1612-56.- The detection of WKK 6219 by Schröder et al. (2009) seems to be the low-velocity horn of J1612-56.

J1616-48A and J1616-48B.-These two H I detections are 2'.0 apart, and the profiles are confused.

J1616-55.- There are ATCA observations on this $\mathrm{HI}$ detection (Staveley-Smith et al. 1998), which shows that the $\mathrm{H}_{\mathrm{I}}$ is extended and has two bright components about $25^{\prime}$ apart. There is no counterpart visible in the optical or NIR despite a moderate extinction $\left(A_{\mathrm{B}}=2 \mathrm{~m} .7\right)$ indicating that the counterpart is likely to have a low surface brightness. Owing to its extent, the coordinates given in previous publications, Henning et al. (2000) and Juraszek et al. (2000), are 11!2 and 10!3 east of our position, while the exact position given in StaveleySmith et al. (1998) is only 5'0 (west) from our position.

J1622-44A.-There is a possible galaxy visible in the NIR at (R.A., decl. $)=(16: 22: 05.2,-44: 22: 36)$ with a distance of 1.5 to the $\mathrm{H}$ i position, which matches the profile well. It lies next to a bright star, which makes the identification uncertain.

J1625-42.-The spectrum also shows J1624-42 at the lower velocities. It appears that the profiles overlap and the line widths and flux measurement are uncertain.

J1625-55.-The cross-match is a galaxy pair: 2MASX $\mathrm{J} 16253644-5533498$ is bright and nearly edge-on (wide profile), while WKK 6819 is a late-type spiral (narrow profile).

J1633-44.- There are two similar-looking small galaxies visible in the NIR, possibly a galaxy pair. The $\mathrm{H}$ I detection is too faint to tell whether one or both galaxies contribute to the profile.

J1648-49.- The H I cross-match seems to be a galaxy pair of similar appearance where both are likely to contribute.

J1651-40.- There is a possible galaxy visible in the NIR at $($ R.A., decl. $)=(16: 51: 53.2,-40: 48: 03)$ with a distance of 1! 1 to the $\mathrm{H}$ i position. WISE shows also a faint reddish patch, though there is a possibility that it is Galactic.

J1653-44.- This galaxy is only visible with WISE and could be also a background galaxy.

J1653-48. - There is a possible galaxy visible in the NIR at $($ R.A., decl. $)=(16: 53: 21.1,-48: 01: 53)$ with a distance of 2.5 to the $\mathrm{H}$ i position, which matches the profile well.

J1705-40.-WISE shows a bright red extended emission at $($ R.A., decl. $)=(17: 05: 04.8,-40: 58: 08)$ with a distance of 1!3 to the H i position. According to the WISE colors, it could be either a starburst galaxy or an H II region (Jarrett et al. 2011).

J1716-42.- The only visible candidate seems too small for this distance, though a larger LSB halo cannot be excluded.

J1716-35.- There are two galaxies visible in the NIR. The edge-on galaxy at (R.A., decl.) $=(17: 16: 35.5,-35: 57: 26)$ does not match the profile as well as the less inclined galaxy at $(17: 16: 51.8,-35: 55: 48)$.

J1719-37.- This galaxy is only visible with WISE and could also be a background galaxy.

J1811-21 and J1812-21.-Both spectra show emission of the other galaxy as well.
J1821-06. - The only candidate appears too small for a velocity of $v=1788 \mathrm{~km} \mathrm{~s}^{-1}$ and $\log M_{\mathrm{H}}=8.8$ even taking the extinction of $A_{\mathrm{B}}=6^{\mathrm{m}}$. 1 into account, but a very LSB halo cannot be excluded.

J1824-20.- This galaxy was found on WISE images and is faintly visible on the IRSF images.

J1825-07.- There are four galaxies visible in the NIR, two of which are NVSS sources and one is an AGN with an optical velocity of $v=10887 \mathrm{~km} \mathrm{~s}^{-1}$ (Burenin et al. 2009). We assume the cross-match to be the most inclined. It is possible that all galaxies belong to a group or cluster.

J1846-07B and J1846-07A.-These two H I detections are only 2.2 apart but well separated in velocity. There are two candidates visible in the NIR: both are very inclined, and the larger is assumed to be the cross-match for J1846-07A, while the cross-match for J1846-07B is smaller and seems to be of later morphological type.

$J 1847+04$. - This galaxy lies at a Galactic latitude of $l=36^{\circ} .06$ and belongs technically to the northern extension of HIZOA (Donley et al. 2005).

J1855-03B.-There is a possible galaxy faintly visible in the NIR at (R.A., decl.) $=(18: 56: 00.5,-03: 12: 21)$ with a distance of 0.3 to the $\mathrm{H}_{\mathrm{I}}$ position. It is large and diffuse and, if real, matches the profile well.

$J 1858+00$. - The profile could be confused or come from a disturbed $\mathrm{H}$ I distribution. The extinction is high $\left(A_{\mathrm{B}}=12 \mathrm{~m} .2\right)$, and only one candidate could be found on the images. No halo is visible, and a hidden galaxy cannot be excluded.

\section{REFERENCES}

Abell, G. O., Corwin, H. G., Jr., \& Olowin, R. P. 1989, ApJS, 70, 1 Acker, A., Stenholm, B., \& Veron, P. 1991, A\&ApS, 87, 499

Alpaslan, M., Robotham, A. S. G., Obreschkow, D., et al. 2014, MNRAS, 440, L106

Alves, M. I. R., Calabretta, M., Davies, R. D., et al. 2015, MNRAS, 450, 2025 Barnes, D. G., Staveley-Smith, L., de Blok, W. J. G., et al. 2001, MNRAS, 322,486

Begum, A., Chengalur, J. N., Karachentsev, I. D., \& Sharina, M. E. 2005, MNRAS, 359, L53

Burenin, R. A., Bikmaev, I. F., Revnivtsev, M. G., et al. 2009, AstL, 35, 71

Calabretta, M. R., Staveley-Smith, L., \& Barnes, D. G. 2014, PASA, 31, e007

Chamaraux, P., \& Masnou, J.-L. 2004, MNRAS, 347, 541

Chamaraux, P., Masnou, J.-L., Kazés, I., et al. 1999, MNRAS, 307, 236

Cluver, M. E., Jarrett, T. H., Appleton, P. N., et al. 2008, ApJL, 686, L17

Cluver, M. E., Jarrett, T. H., Kraan-Korteweg, R. C., et al. 2010, ApJ, 725,1550

Corwin, H. G., de Vaucouleurs, A., \& de Vaucouleurs, G. 1985, University of Texas Monographs in Astronomy (Austin: Univ. Texas), 1985

Courtois, H. M., Hoffman, Y., Tully, R. B., \& Gottlöber, S. 2012, ApJ, 774, 43 de Lapparent, V., Geller, M. J., \& Huchra, J. P. 1986, ApJL, 302, L1

Donley, J. L., Koribalski, B. S., Staveley-Smith, L., et al. 2006, MNRAS, 369,1741

Donley, J. L., Staveley-Smith, L., Kraan-Korteweg, R. C., et al. 2005, AJ, 129,220

Ebeling, H., Kocevski, D., Tully, R. B., \& Mullis, C. R. 2005, in ASP Conf. Ser. 329, Nearby Large-Scale Structures and the Zone of Avoidance, ed. A. P. Fairall \& P. A. Woudt (San Francisco, CA: ASP), 83

Ebeling, H., Mullis, C. R., \& Tully, R. B. 2002, ApJ, 580, 774

Erdog`du, P., Lahav, O., Huchra, J. P., et al. 2006, MNRAS, 373, 45

Fairall, A. P. 1980, MNRAS, 192, 389

Fairall, A. P. 1998, Large-scale Structures in the Universe (Praxis: Wiley)

Fairall, A. P., Woudt, P. A., \& Kraan-Korteweg, R. C. 1998, A\&AS, 127, 463

Gooch, R. 1996, adass V, 101, 80

Hasegawa, T., Wakamatsu, K.-i., Malkan, M., et al. 2000, MNRAS, 316, 326 Helou, G., \& Walker, D. W. 1988, Infrared Astronomical Satellite (IRAS) Catalogs and Atlases, Vol. 7, 1

Henning, P. A., Kraan- orteweg, R., Staveley-Smith, L., et al. 2005, in ASP Conf. Ser. 329, Nearby Large-Scale Structures and the Zone of Avoidance, ed. A. P. Fairall \& P. A. Woudt (San Francisco, CA: ASP), 199 
Henning, P. A., Springob, C. M., Minchin, R. F., et al. 2010, AJ, 139, 2130 Henning, P. A., Staveley-Smith, L., Ekers, R. D., et al. 2000, AJ, 119, 2686 (HIZSS)

Hoppmann, L., Staveley-Smith, L., Freudling, W., et al. 2015, MNRAS, 452, 3726

Huchra, J. P., Macri, L. M., Masters, K. L., et al. 2012, ApJS, 199, 26

Huchtmeier, W. K., Karachentsev, I. D., \& Karachentseva, V. E. 2001, A\&A, 377,801

Jarrett, T. H., Chester, T., Cutri, R., et al. 2000, AJ, 119, 2498

Jarrett, T. H., Cohen, M., Masci, F., et al. 2011, ApJ, 735, 112

Jarrett, T. H., Koribalski, B. S., Kraan-Korteweg, R. C., et al. 2007, AJ, 133,979

Johnston, R., Teodoro, L., \& Hendry, M. 2007a, MNRAS, 376, 1757

Johnston, S., Bailes, M., Bartel, N., et al. 2007b, PASA, 24, 174

Juraszek, S. J., Staveley-Smith, L., Kraan-Korteweg, R. C., et al. 2000, AJ, 119,1627

Kerp, J., Winkel, B., Ben Bekhti, N., Flöer, L., \& Kalberla, P. M. W. 2011, AN, 332, 637

Kerr, F. J., \& Henning, P. A. 1987, ApJL, 320, L99

Kilborn, V. A., Webster, R. L., Staveley-Smith, L., et al. 2002, AJ, 124, 690

Koribalski, B. S. 2012, PASA, 29, 359

Koribalski, B. S., Staveley-Smith, L., Kilborn, V. A., et al. 2004, AJ, 128, 16

Kraan-Korteweg, R. C. 1989, Astronomische Gesellschaft Abstract Series, 2,54

Kraan-Korteweg, R. C. 2000, A\&AS, 141, 123

Kraan-Korteweg, R. C. 2005, RvMA, 18, 48

Kraan-Korteweg, R. C., Cayatte, V., Balkowski, C., Fairall, A. P., \& Henning, A. P. 1994, in ASP Conf. Ser. 67, Unveiling Large-scale Structures behind the Milky Way, ed. C. Balkowski \& R. C. Kraan-Korteweg (San Francisco, CA: ASP), 99

Kraan-Korteweg, \& Woudt 1994, in ASP Conf. Ser. 67, Unveiling Large-Scale Structures Behind the Milky Way, ed. C. Balkowski \& R. C. Kraan-Korteweg (San Francisco, CA: ASP), 89

Kraan-Korteweg, R. C., Fairall, A. P., \& Balkowski, C. 1995, A\&A, 297, 617

Kraan-Korteweg, R. C., Henning, P. A., \& Schröder, A. C. 2002, A\&A, 391,887

Kraan-Korteweg, R. C., \& Huchtmeier, W. K. 1992, A\&A, 266, 150

Kraan-Korteweg, R. C., \& Lahav, O. 2000, A\&ARv, 10, 211

Kraan-Korteweg, R. C., Riad, I. F., Woudt, P. A., Nagayama, T., \& Wakamatsu, K. 2011, Ten Years of IRSF-and the Future (Nagoya: Nagoya Univ.)

Kraan-Korteweg, R. C., Staveley-Smith, L., Donley, J., Koribalski, B., \& Henning, P. A. 2005, Maps of the Cosmos, 216, 203

Kraan-Korteweg, R. C., \& Woudt, P. A. 1994, in Unveiling Large-scale Structures behind the Milky Way 67, 89

Kraan-Korteweg, R. C., \& Woudt, P. A. 1999, PASA, 16, 53

Kraan-Korteweg, R. C., Woudt, P. A., Cayatte, V., et al. 1996, Natur, 379, 519

Kraan-Korteweg, Renée, C., Shafi, N., Koribalski, B. S., \& Staveley-Smith, L. 2008, in Galaxies in the Local Volume, ed. H. Jerjen \& B. S. Koribalski (Netherlands: Springer), 13

Lavaux, G., \& Hudson, M. J. 2011, MNRAS, 416, 2840

Lavaux, G., Tully, R. B., Mohayaee, R., \& Colombi, S. 2010, ApJ, 709, 483

Loeb, A., \& Narayan, R. 2008, MNRAS, 386, 2221

Ma, Y.-Z., Branchini, E., \& Scott, D. 2012, MNRAS, 425, 2880

Massey, P., Henning, P. A., \& Kraan-Korteweg, R. C. 2003, AJ, 126, 2362

Matthews, L. D., \& Gallagher, J. S., III 1996, AJ, 111, 1098

Matthews, L. D., Gallagher, J. S., III, \& Littleton, J. E. 1995, AJ, 110, 581

McIntyre, T. P., Henning, P. A., Minchin, R. F., Momjian, E., \& Butcher, Z. 2015, AJ, 150, 28

Meyer, M. J., Zwaan, M. A., Webster, R. L., et al. 2004, MNRAS, 350, 1195

Mullis, C. R., Ebeling, H., Kocevski, D. D., \& Tully, R. B. 2005, in ASP Conf. Ser. 329, Nearby Large-Scale Structures and the Zone of Avoidance, ed. A. P. Fairall \& P. A. Woudt (San Francisco, CA: ASP), 183
Nagayama, T., Woudt, P. A., Nagashima, C., et al. 2004, MNRAS, 354, 980 Paturel, G., Petit, C., Prugniel, P., et al. 2003, A\&A, 412, 45

Radburn-Smith, D. J., Lucey, J. R., Woudt, P. A., Kraan-Korteweg, R. C., \& Watson, F. G. 2006, MNRAS, 369, 1131

Ramatsoku, M., Kraan-Korteweg, R. C., Schröder, A. C., \& van Driel, W. 2014, arXiv: 1412.5324

Rauzy, S. 2001, MNRAS, 324, 51

Ryan-Weber, E., Koribalski, B. S., Staveley-Smith, L., et al. 2002, AJ, 124, 1954

Said, K., Kraan-Korteweg, R. C., \& Jarrett, T. H. 2015, MNRAS, 447, 1618

Sault, R. J., Teuben, P. J., \& Wright, M. C. H. 1995, adass IV, 77, 433

Saunders, W., Sutherland, W. J., Maddox, S. J., et al. 2000, MNRAS, 317,55

Schlafly, E. F., \& Finkbeiner, D. P. 2011, ApJ, 737, 103

Schlegel, D. J., Finkbeiner, D. P., \& Davis, M. 1998, ApJ, 500, 525

Schröder, A. C., Kraan-Korteweg, R. C., \& Henning, P. A. 2009, A\&A, 505, 1049

Schröder, A. C., Mamon, G. A., Kraan-Korteweg, R. C., \& Woudt, P. A. 2007, A\&A, 466, 481

Shafi, N. 2008, M.Sc. thesis, Univ. Cape Town

Springob, C. M., Magoulas, C., Colless, M., et al. 2014, MNRAS, 445,2677

Staveley-Smith, L. 1997, PASA, 14, 111

Staveley-Smith, L., Juraszek, S., Koribalski, B. S., et al. 1998, AJ, 116, 2717

Staveley-Smith, L., Wilson, W. E., Bird, T. S., et al. 1996, PASA, 13, 243

Stein, P. 1997, A\&A, 317, 670

Tully, R. B., \& Fisher, J. R. 1987, Atlas of Nearby Galaxies (Cambridge: Cambridge University Press)

Tully, R. B., Rizzi, L., Shaya, E. J., et al. 2009, AJ, 138, 323

van Driel, W., Schneider, S. E., Kraan-Korteweg, R. C., \& Monnier Ragaigne, D. 2009, A\&A, 505, 29

Verheijen, M., Oosterloo, T., Heald, G., \& van Cappellen, W. 2009, in Panoramic Radio Astronomy: Wide-field 1-2 GHz Research on Galaxy Evolution, 10

Visvanathan, N., \& Yamada, T. 1996, ApJS, 107, 521

Wakamatsu, K., Malkan, M. A., Nishida, M. T., et al. 2005, ASP Conf. Ser. 329, Nearby Large-Scale Structures and the Zone of Avoidance, ed. A. P. Fairall \& P. A. Woudt (San Francisco, CA: ASP), 189

Watkins, R., Feldman, H. A., \& Hudson, M. J. 2009, MNRAS, 392, 743

Williams, W. L., Kraan-Korteweg, R. C., \& Woudt, P. A. 2014, MNRAS, 443, 41

Wong, O. I., Ryan-Weber, E. V., Garcia-Appadoo, D. A., et al. 2006, MNRAS, 371, 1855

Woudt, P. A., \& Kraan-Korteweg, R. C. 2000, in ASP Conf. Ser. 218, Mapping the Hidden Universe: The Universe Behind the Milky Way-The Universe in Hi, ed. R. C. Kraan-Korteweg, P. A. Henning \& H. Andernach (San Francisco, CA: ASP), 193

Woudt, P. A., \& Kraan-Korteweg, R. C. 2001, A\&A, 380, 441

Woudt, P. A., Kraan-Korteweg, R. C., Cayatte, V., Balkowski, C., \& Felenbok, P. 2004, A\&A, 415, 9

Woudt, P. A., Kraan-Korteweg, R. C., \& Fairall, A. P. 1999, A\&A, 352, 39

Woudt, P. A., Kraan-Korteweg, R. C., Fairall, A. P., et al. 2000, in ASP Conf. Ser. 218, Mapping the Hidden Universe: The Universe behind the Milky Way-The Universe in HI, ed. R. C. Kraan-Korteweg, P. A. Henning \& H. Andernach (San Francisco, CA: ASP), 203

Woudt, P. A., Kraan-Korteweg, R. C., Lucey, J., Fairall, A. P., \& Moore, S. A. W. 2008, MNRAS, 383, 445

Wright, A., \& Otrupcek, R. 1990, PKS Catalog, 1990, 0

Wright, E. L., Eisenhardt, P. R. M., Mainzer, A. K., et al. 2010, AJ, 140,1868

Zwaan, M. A., Meyer, M. J., Webster, R. L., et al. 2004, MNRAS, 350, 1210 